Characteristics of Identifying Linear Dynamic Models from Impulse Response Data Using Prony Analysis

D. J. Trudnowski

December 1992

Prepared for

the Bonneville Power Administration under a Related Services Agreement with the U.S. Department of Energy

Contract DE-AC06-76RLO 1830

Pacific Northwest Laboratory

Operated for the U.S. Department of Energy by Battelle Memorial Institute 


\title{
DISCLAIMER
}

This report was prepared as an account of work sponsored by an agency of the United States Government. Neither the United States Government nor any agency thereof, nor Battelle Memorial Institute, nor any of their employees, makes any warranty, expressed or implied, or assumes any legal liability or responsibility for the accuracy, completeness, or usefulness of any information, apparatus, product, or process disclosed, or represents that its use would not infringe privately owned rights. Reference herein to any specific commercial product, process, or service by trade name, trademark, manufacturer, or otherwise does not necessarily consittute or imply its endorsement, recommendation, or favoring by the United States Government or any agency thereof, or Battelle Memorial Institute. The views and opinions of authors expressed herein do not necessarily state or reflect those of the United States Government or any agency thereof.

\author{
PACIFIC NORTHWEST LABORATORY \\ operated by \\ BATTELLE MEMORIAL INSTITUTE \\ for the \\ UNITED STATES DEPARTMENT OF ENERGY \\ under Contract DE-AC06-76RLO 1830
}

Printed in the United States of America

Available to DOE and DOE contractors from the

Office of Scientific and Technical Information, P.O. Box 62, Oak Ridge, TN 37831;

prices available from (615) 576-8401. FTS 626-8401.

Available to the public from the National Technical Information Service,

U.S. Department of Commerce, 5285 Port Royal Rd., Springfield, VA 22161. 


\section{Characteristics of \\ Identifying Linear Dynamic Models \\ from Impulse Kesponse Data \\ Using Prony Analysis}

D. J. Trudnowski

December 1992

Prepared for

the Bonneville Power Administration under a Related Services Agreement with the U.S. Department of Energy

Contract DE-AC06-76RLO 1830

Pacific Northwest Laboratory

Richland, Washington 99352

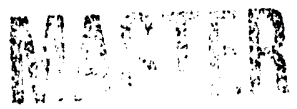




\section{Summary}

Pacific Northwest Labciutory (PNL) conducted this study for the Bonneville Power Administration (BPA). The purpose of the study was to investigate the characteristics of fitting linear dynamic models to the impulse response of oscillatory dynamic systems using Prony analysis.

Many dynamic systems exhibit oscillatory re.inonses with multiple modes of oscillations. Although the underlying dynamics of such systems are often nonlinear, it is frequently possible and very useful to represent the system operating about some set point with a linear model. Derivation of such linear models can be done using two basic approaches: model the system using theoretical derivations and some linearization method such as a Taylor series expansion; or use a curve-fitting technique to optimally fit a linear model to specified system response data. Prony analysis belongs to the second class of system modeling because it is a method of fitting a linear model to the impulse response of a dynamic system. Its parallel formulation inherently makes it well suited for fitting models to oscillatory system data.

Such oscillatory dynamic effects occur in large synchronous-generator-based power systems in the form of electromechanical oscillations. To study and characterize these oscillatory dynamics, BPA has developed computer codes to analyze system data using Prony analysis. The objective of this study was to develop a highly detailed understanding of the properties of using Prony analysis to fit models to systems with characteristics often encountered in power systems. This understanding was then extended to develop general "rules-of-thumb" for using Prony analysis.

The general characteristics were investigated by performing fits to data from known linear models under controlled conditions. The conditions studied include various mathematical solution techniques; different parent system configurations; and a large variety of underlying noise characteristics. Some of the general guidelines for intelligent use of Prony analysis include the following:

- In sampling the system data, use a sample period at least double the Nyquist rate but not more than 10 times the Nyquist rate.

- In choosing the length of the data record, several factors must be considered: 1) at least one full cycle of the minimum-frequency oscillatory mode should be included (more data is preferred); 2) if data is collected in a noisy environment, an excessively long data record should be avoided (this is somewhat a judgment, but the record should be short enough to keep the signal-to-noise ratio (SNR) above $20 \mathrm{db}$ ); 3) if a double-precision root finder is employed in solving the Prony analysis problem, the number of data points should not exceed 250 (a safer limit is 100); and 4) for a quad-precision root finder, the limits are 500 and 200.

- In most cases, one should not expect to accurately identify stable real poles with a magnitude greater than $1 /(3 T)$, where $T$ is the sample period.

- Use the covariance method in the forward direction to formulate the linear prediction (LP) problem for the Prony analysis solution. Also, if possible, use a quad-precision root finder 
to factor the LP polynomial.

- In choosing the order of the LP fit, use $N / 3$ to $N / 2$ (where $N$ is the number of data points), as long as it does not exceed 50 for a double-precision root finder or 100 for a quad.

- Accurate fits are likely if the SNR is $20 \mathrm{db}$ or greater. If the noise is highly colored, poor fits are likely for poles and residues in the bandwidth of the colored noise.

- If closely-spaced poles are identified, especially with similar imaginary terms, the error bounds for these terms are likely to be large even if the fit is graphically accurate. 


\section{Contents}

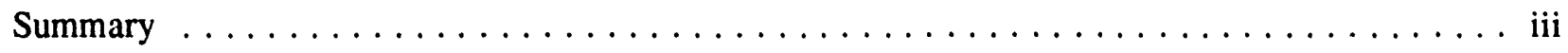

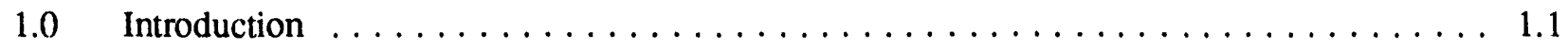

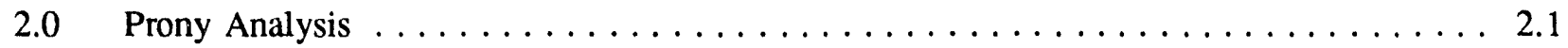

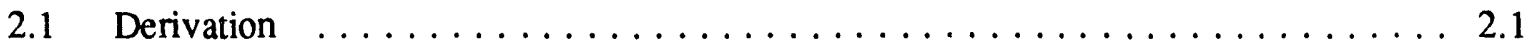

$2.2 \quad$ Literature Review $\ldots \ldots \ldots \ldots \ldots \ldots \ldots \ldots \ldots \ldots \ldots \ldots \ldots, 2.3$

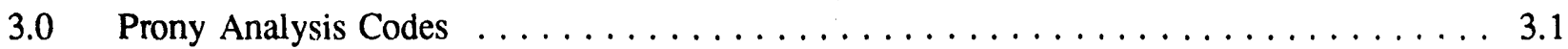

$3.1 \quad$ SIGPAKZ and IPRONY $\ldots \ldots \ldots \ldots \ldots \ldots \ldots \ldots \ldots \ldots \ldots \ldots \ldots \ldots \ldots \ldots \ldots$

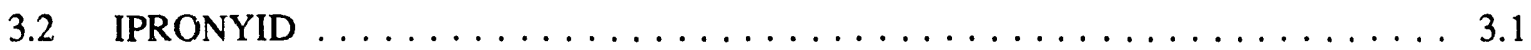

$3.3 \quad$ PRSPAK (Core Prony Subroutine) $\ldots \ldots \ldots \ldots \ldots \ldots \ldots \ldots \ldots \ldots \ldots \ldots \ldots \ldots \ldots \ldots .2$

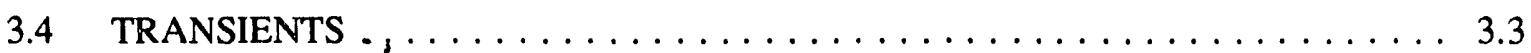

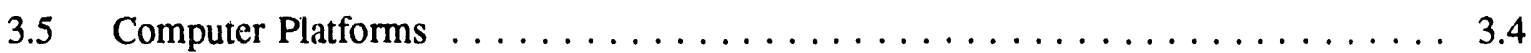

4.0 Example Systems and MATLAB Interfaces $\ldots \ldots \ldots \ldots \ldots \ldots \ldots \ldots \ldots \ldots \ldots \ldots$

$4.1 \quad$ Interfacing with MATLAB $\ldots \ldots \ldots \ldots \ldots \ldots \ldots \ldots \ldots \ldots \ldots \ldots \ldots \ldots \ldots \ldots$

$4.2 \quad$ Test Systems $\ldots \ldots \ldots \ldots \ldots \ldots \ldots \ldots \ldots \ldots \ldots \ldots \ldots \ldots \ldots .2$

$4.2 .1 \quad$ Test System $1 \ldots \ldots \ldots \ldots \ldots \ldots \ldots \ldots \ldots \ldots \ldots \ldots .2$

4.2.2 Test System $2 \ldots \ldots \ldots \ldots \ldots \ldots \ldots \ldots \ldots \ldots \ldots \ldots \ldots . \ldots \ldots \ldots$

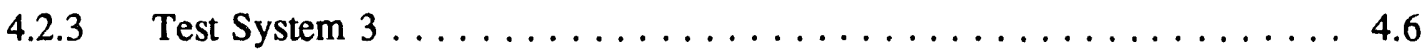

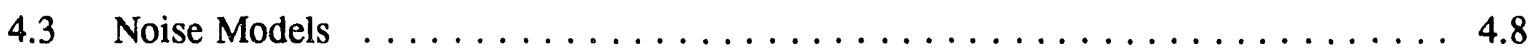

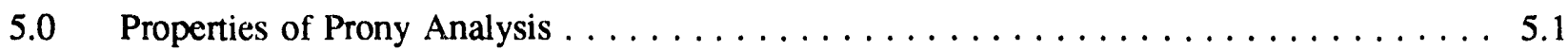

5.1 Alternative Solution Techniques $\ldots \ldots \ldots \ldots \ldots \ldots \ldots \ldots \ldots \ldots .1$

5.1.1 The Linear Prediction Method $\ldots \ldots \ldots \ldots \ldots \ldots \ldots \ldots \ldots . .2$

5.1.2 Linear Prediction Algorithm $\ldots \ldots \ldots \ldots \ldots \ldots \ldots \ldots \ldots \ldots . \ldots \ldots .2$ 
5.1.3 Forward/Backward Formulation $\ldots \ldots \ldots \ldots \ldots \ldots \ldots \ldots$

$5.1 .4 \quad$ Vandermonde System-Solving Algorithms $\ldots \ldots \ldots \ldots \ldots \ldots . \ldots . \ldots$

5.2 Effects of Linear Prediction Model Order $\ldots \ldots \ldots \ldots \ldots \ldots \ldots \ldots \ldots$

5.3 Effects of Sampling Frequency $\ldots \ldots \ldots \ldots \ldots \ldots \ldots \ldots \ldots \ldots$

5.4 Effects of Record Length $\ldots \ldots \ldots \ldots \ldots \ldots \ldots \ldots \ldots \ldots \ldots \ldots$

5.5 Effects of Noise Level and Coloration $\ldots \ldots \ldots \ldots \ldots \ldots \ldots \ldots \ldots \ldots$

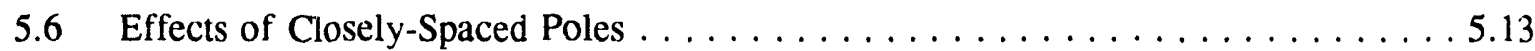

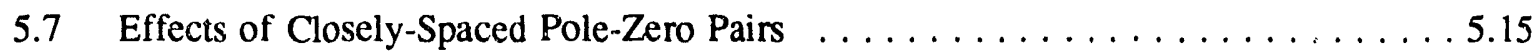

6.0 Conclusions and Recommendations $\ldots \ldots \ldots \ldots \ldots \ldots \ldots \ldots \ldots \ldots \ldots \ldots$

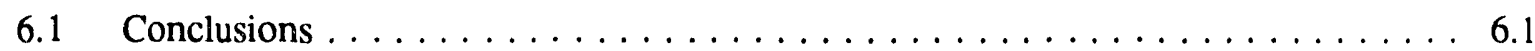

6.2 Recommended Future Work $\ldots \ldots \ldots \ldots \ldots \ldots \ldots \ldots \ldots \ldots \ldots . \ldots \ldots$

$6.2 .1 \quad$ Overhaul PSRPAK $\ldots \ldots \ldots \ldots \ldots \ldots \ldots \ldots \ldots \ldots . \ldots \ldots \ldots$

6.2.2 Implement Revision Control System . . . . . . . . . . . . . . . . . 6.4

6.2.3 Incorporate Transfer Function Identification into SIGPAKZ . . . . . . . . 6.4

6.2.4 Develop "Local Intelligence" in SIGPAKZ and IPRONYID . . . . . . . . 6.4

6.2.5 Investigate New Options $\ldots \ldots \ldots \ldots \ldots \ldots \ldots \ldots \ldots \ldots$

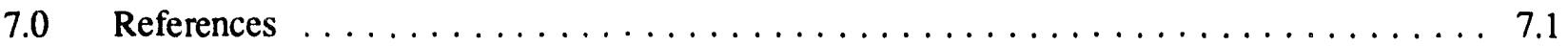

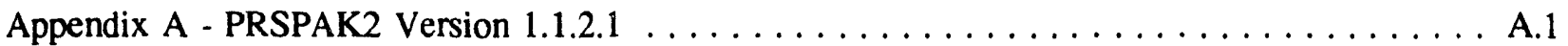

Appendix B - Section 5 SIGPAKZ Input Files $\ldots \ldots \ldots \ldots \ldots \ldots \ldots \ldots \ldots \ldots$

Appendix $\mathrm{C}$ - TRANFUN and ORDER Subroutines $\ldots \ldots \ldots \ldots \ldots \ldots \ldots \ldots \ldots$ 


\section{Figures}

4.1 Impulse response of test system $1 \ldots \ldots \ldots \ldots \ldots \ldots \ldots \ldots \ldots \ldots \ldots$

$4.2 \quad$ Frequency response of test system $1 \ldots \ldots \ldots \ldots \ldots \ldots \ldots \ldots \ldots \ldots$

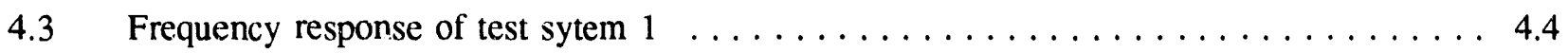

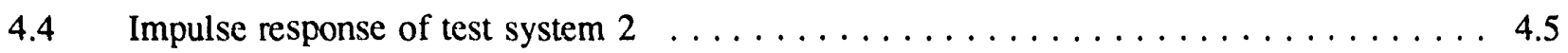

4.5 Frequency response of test system $2 \ldots \ldots \ldots \ldots \ldots \ldots \ldots \ldots \ldots \ldots$

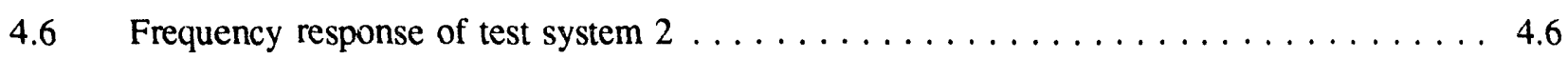

$4.7 \quad$ Impulse response of test system $3 \ldots \ldots \ldots \ldots \ldots \ldots \ldots \ldots \ldots \ldots$

$4.8 \quad$ Frequency response of test system $3 \ldots \ldots \ldots \ldots \ldots \ldots \ldots \ldots \ldots \ldots$

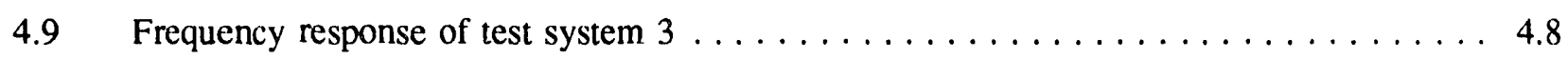

4.10 Frequency response of $1 / f$ and $1 / f^{2}$ filters $\ldots \ldots \ldots \ldots \ldots \ldots \ldots \ldots$

4.11 Frequency response of coloring filter $\ldots \ldots \ldots \ldots \ldots \ldots \ldots \ldots \ldots \ldots \ldots \ldots \ldots \ldots$

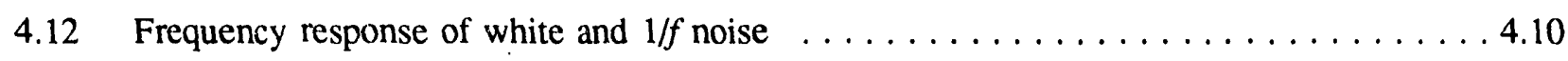

4.13 Frequency response of white and $1 / f^{\sharp}$ noise $\ldots \ldots \ldots \ldots \ldots \ldots \ldots \ldots \ldots \ldots$

4.14 Frequency response of colored noise $\ldots \ldots \ldots \ldots \ldots \ldots \ldots \ldots \ldots \ldots \ldots \ldots \ldots$

Impulse response of 3 rd-order fit to 9 th-order system with
white noise at $27-\mathrm{db}$ SNR, Example $5.3 \ldots \ldots \ldots \ldots \ldots \ldots \ldots \ldots \ldots \ldots$

5.2 Frequency response of 3 rd-order fit to 9 th-order system with
white noise at 27 -db SNR, Example $5.3 \ldots \ldots \ldots \ldots \ldots \ldots \ldots \ldots \ldots \ldots \ldots \ldots$

5.3 Impulse response of 15 th-order fit to 9 th-order system with white noise at $27-\mathrm{db}$ SNR, Example $5.3 \ldots \ldots \ldots \ldots \ldots \ldots \ldots$

5.4 Frequency response of 15 th-order fit to 9 th-order system with white noise at $27-\mathrm{db}$ SNR, Example $5.3 \ldots \ldots \ldots \ldots \ldots \ldots$

5.5 Impulse response of 30th-order fit to 9th-order system with white noise at $27-\mathrm{db}$ SNR, Example $5.3 \ldots \ldots \ldots \ldots \ldots \ldots \ldots \ldots$ 
5.6 Frequency response of 30th-order fit to 9th-order system with white noise at $27-\mathrm{db}$ SNR, Example $5.3 \ldots \ldots \ldots \ldots \ldots \ldots \ldots \ldots$

5.7 Different data record fits impulse response, Example $5.4 \ldots \ldots \ldots \ldots \ldots$

5.8 Different record length fits frequency response, Example $5.4 \ldots \ldots \ldots \ldots \ldots \ldots$

5.9 Impulse response of different record length fits, Exarnple $5.5 \ldots \ldots \ldots \ldots \ldots$

5.10 Frequency response of different record length fits, Example $5.5 \ldots \ldots \ldots \ldots . \ldots$

5.11 Impulse response of fit to system with $40-\mathrm{db}$ SNR, Example $5.6 \ldots \ldots \ldots \ldots \ldots .11$

5.12 Impulse response of fit to system with $15-\mathrm{db}$ SNR, Example $5.6 \ldots \ldots \ldots \ldots . \ldots \ldots$

5.13 Frequency response of different noise level fits, Example $5.6 \ldots \ldots \ldots \ldots \ldots \ldots$

5.14 Impulse response of system $(5.5)$, Example $5.8 \ldots \ldots \ldots \ldots \ldots \ldots \ldots \ldots \ldots \ldots \ldots$

5.15 Frequency response of system $(5.5)$, Example $5.8 \ldots \ldots \ldots \ldots \ldots \ldots \ldots$

5.16 Impulse response of the $p=0.9$ fit, Example $5.8 \ldots \ldots \ldots \ldots \ldots \ldots \ldots \ldots$

5.17 Frequency response of the $p=0.9$ fit, Example $5.8 \ldots \ldots \ldots \ldots \ldots \ldots$

5.18 Impulse response of system (5.6), Example $5.9 \ldots \ldots \ldots \ldots \ldots \ldots \ldots$

5.19 Frequency response of system (5.6), Example $5.9 \ldots \ldots \ldots \ldots \ldots \ldots$ 


\section{Tables}

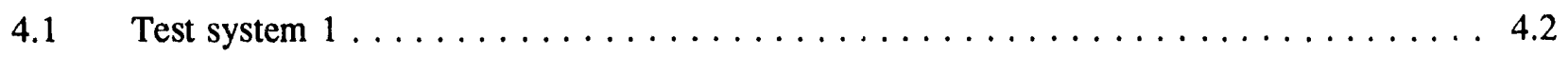

$4.2 \quad$ Test system $2 \ldots \ldots \ldots \ldots \ldots \ldots \ldots \ldots \ldots \ldots \ldots \ldots \ldots \ldots \ldots \ldots$

$4.3 \quad$ Test system $3 \ldots \ldots \ldots \ldots \ldots \ldots \ldots \ldots \ldots \ldots \ldots \ldots \ldots \ldots \ldots$

$5.1 \quad$ Identified poles for given LP methods, Example $5.1 \ldots \ldots \ldots \ldots \ldots \ldots$

5.2 Identified $0.35-\mathrm{hz}$ terms for forward/backward formulations, Example $5.2 \ldots \ldots .3$

5.3 Identified poles and residues for given colored noise, Example $5.7 \ldots \ldots \ldots \ldots$

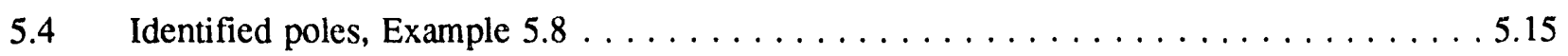

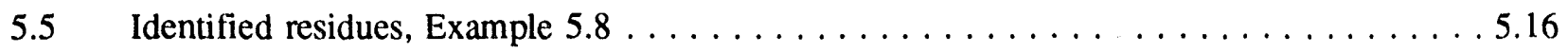

$5.6 \quad$ Identified poles for closely-spaced pole-zero pairs, Example $5.9 \ldots \ldots \ldots \ldots$

5.7 Identified residues for closely-space pole-zero pairs, Exam ${ }_{2} 5.9 \ldots \ldots \ldots \ldots$ 


\subsection{Introduction}

Many dynamic systems exhibit oscillatory responses with multiple modes of oscillations. Examples include electromechanical oscillations in large synchronous-generator-based power systems and flexible mechanical structures and manipulators. Although the underlying dynamics of such systems are often nonlinear, it is often possible and very useful to represent the system operating about some set point with a linear model. Derivation of such linear models can be done using two basic approaches: model the system using theoretical derivations and some linearization method such as a Taylor series expansion; or use a curve-fitting technique to optimally fit a linear model to specified system response data. Prony analysis belongs to the second class of system modeling because it is a method of fitting a linear model to the impulse response of a dynamic system. Its parallel formulation inherently makes it well suited for fitting models to oscillatory system data.

Such oscillatory dynamic effects occur in large synchronous-generator-based power systems in the form of electromechanical oscillations. These oscillations arise when energy is exchanged between generators, or sets of generators, through transmission lines. In theory, a power system has $m-1$ modes of oscillation, where $m$ is the number of generators; modern systems have hundreds of generators. In reality, any one signal will be dominated by a few modes. To study and characterize these oscillatory dynamics, the Bonneville Power Administration (BPA) has developed computer codes to analyze system data using Prony analysis. To make full integrated use of the Prony analysis codes, as well as other analysis techniques, BPA has commissioned Pacific Northwest Laboratory (PNL) ${ }^{(a)}$ to further develop the codes and techniques into a software tool termed the Power System Identification (PSI) Toolbox.

The first phase of the PSI Toolbox development involves investigating the characteristics of the analysis techniques by evaluating existing software and developing guidelines for best use.

Subsequent phases include extending current software; developing new analysis algorithms; and reorganizing the software into a modular collection of computer programs.

The primary focus of this report falls under the first phase. The characteristics of using Prony analysis to fit a linear model to the impulse response of an oscillatory system are investigated. The investigation is conducted by performing fits to the impulse response of known linear models under controlled conditions. The conditions studied include fitting algorithms, parent system configurations, and underlying noise characteristics. Many general "rules of thumb" for fitting under these conditions are drawn from the testing results. Special effort is made to investigate the effects that might be encountered in studying electromechanical oscillations in power systems. These oscillations typically occur in the $0.1-\mathrm{hz}$ to $2.0-\mathrm{hz}$ range; therefore, examples systems used in the investigations consist of multiple modes in this frequency range. Although the intended focus of this work is power system osciliations, the studies are generic enough that the results are applicable to many types of oscillatory. systems with closely-spaced modes (including flexible mechanical structures and manipulators).

The following section focuses on introducing Prony analysis and presenting a literature review on associated numerical fitting algorithms. The different Prony analysis computer codes used to conduct

(a) Pacific Northwest Laboratory is operated by Battelle Memorial Institute for the U.S. Department of Energy under Contract DE-AC06-76RLO 1830. 
tests are discussed in Section 3. In Section 4, test systems and noise models are presented. The main results on testing Prony analysis under different conditions are presented in Section 5, and conclusions and recommendations are discussed in Section 6. 


\subsection{Prony Analysis}

Prony analysis is a numerical technique that fits a ighted summation of exponential terms to a general signal. It provides modal, damping, phase, and inagnitude information contained in the signal. A complete and up-to-date review of Prony analysis is contained in Scharf (1991). The technique originated nearly 200 years ago (Prony 1795), but only with the advancement of the modern digital computer has its full implementation become feasible. In this section, Prony analysis is first introduced, followed by a review of literature focussed on numerical algorithms for solving the Prony analysis problem.

\subsection{Derivation}

Prony analysis provides a method of fitting a signal to a weighted linear combination of exponential terms. Corsider a general signal $y(t)$ that is to be modeled by

$$
\hat{y}(t)=\sum_{i=1}^{n} B_{i} e^{\lambda_{i}}
$$

where $B_{i} \in \mathbb{C}$ is termed the output residue for the continuous-time pole $\lambda_{i} \in \mathbb{C}$. The objective is to identify the residues, poles, and $n$ that force the signal of $(2.1)$ to be the least-squares error fit to $y(t)$.

The signal $y(t)$ is sampled at a constant sample period $(T)$ smaller than the Nyquist period, and

$a$ is rewritten in discrete-time form as

$$
\hat{y}_{k}=\sum_{i=1}^{n} B_{i} z_{i}^{k}, \quad k=0,1, \ldots, N-1
$$

where

$$
z_{i}=e^{\lambda_{1} T}
$$

$z_{i} \in \mathbf{C}$ is termed the discrete-time pole. It is not required that the original signal be a continuous set. One can just as easily start with evenly-spaced discrete data and formulate the starting model as (2.2).

It is well known that the output of (2.2) satisfies its own characteristic equation. Let the characteristic equation of (2.2) be written as

$$
p(z)=z^{n}-\left(a_{1} z^{n-1}+a_{2} z^{n-2}+\ldots+a_{n}\right)
$$

Then the output of (2.2) satisfies

$$
\hat{y}_{k}=a_{1} \hat{y}_{k-1}+a_{2} \hat{y}_{k-2}+\ldots+a_{n} \hat{y}_{k-n}
$$

for $k=0,1, \ldots, N-1$. Equation (2.5) is known as an auto-regressive (AR) sequence written in the forward 
direction. Assuming $\hat{y}$ is a real function, it is also well known that it satisfies

$$
\hat{y}_{k}=\bar{a}_{1} \hat{y}_{i+1}+\bar{a}_{2} \hat{y}_{k+2}+\ldots+\bar{a}_{n} \hat{y}_{k+n}
$$

for $k=0,1, \ldots, N-1$, and where the roots of the polynomial

$$
\bar{p}(z)=1-\left(\bar{a}_{1} z+\bar{a}_{2} z^{2}+\ldots+\bar{a}_{n} z^{n}\right)
$$

are reflections about the unit circle of the roots of the polynomial in (2.4). Equation (2.6) is the AR sequence written in the backward direction.

Generally, the process of solving for the unknowns is conducted in three steps:

1. $y$ is substituted for $\hat{y}$ in (2.5) and/or (2.6), and the linear equations are solved for the unknown $a_{i}$ 's. This step is termed the Linear Prediction (LP) problem.

2. Polynomials (2.4) and/or (2.7) are factored for the discrete-time poles (i.e., the $z_{i}$ 's). This, in turn, yields the continuous-time poles.

3. Again, substituting $y$ for $\hat{j},(2.2)$ is solved for the output residues. Because (2.2) involves a Vandermonde matrix, this is termed the Vandermonde problem.

Solving step 1 has received the most attention in the literature. The problem can be formulated many different ways. For example, consider only the forward direction problem. If initial (i.e., $k<0$ ) and post (i.e., $k>N$ ) conditions are assumed zero, then the equations (2.5) are written as

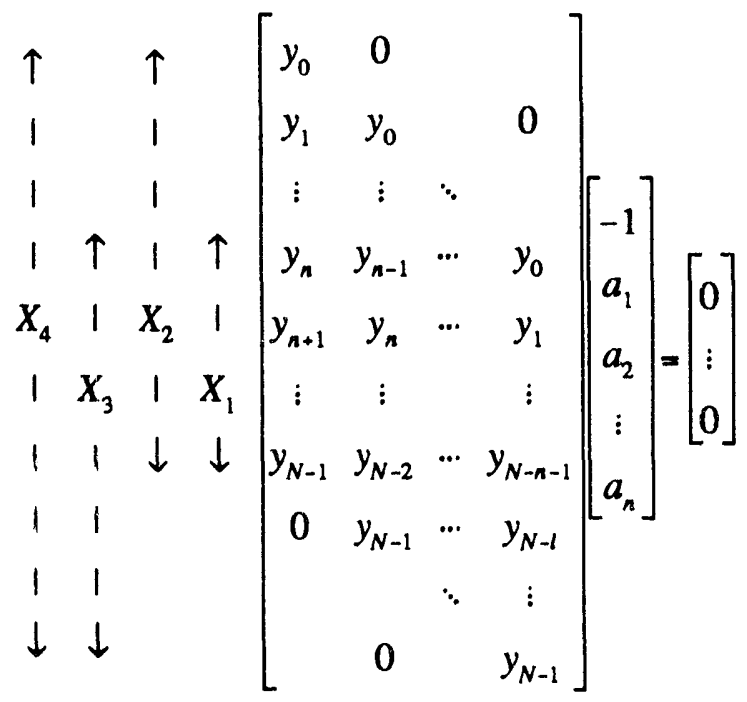

The matrix in (2.8) is the Toeplitz data matrix. One could just as easily write the analogous matrix equation using (2.6), or a combination of (2.5) and (2.6). In the majority of practical cases the Toeplitz rnatrix is non-square with more rows than columns. Then a least-squares solution can be calculated by minimizing the right-hand side of $(2.8)$. 
Four different formulations are indicated in (2.8); these are $X_{1}$ through $X_{4} . X_{1}$ is termed the covariance problem. Because initial and post conditions are not used, no assumption is required on their value. $X_{4}$ is the correlation problem; it incorporates both initial and post conditions. The last two problems, $X_{2}$ and $X_{3}$, are termed the pre-windowed and post-windowed methods.

The solution to step 2 can be done in one of two ways: eigenanalysis or polynomial factoring. With eigenanalysis, a companion matrix is constructed for polynomials (2.4) and/or (2.7) and the eigenvalues whick are equal to the $z_{i}$ 's are calculated. In using polynomial factoring, the roots of (2.4) and/or (2.7) are calculated using a root-finding algorithm.

Step 3 is also a linear algebra problem. Once the $z_{i}$ 's are obtained from step 2, they are substituted into (2.2) and written in matrix form as

$$
\left[\begin{array}{cccc}
1 & 1 & \cdots & 1 \\
z_{1} & z_{2} & \cdots & z_{n} \\
z_{1}^{2} & z_{2}^{2} & \cdots & z_{n}^{2} \\
\vdots & \vdots & \vdots & \vdots \\
z_{1}^{N-1} & z_{2}^{N-1} & \cdots & z_{n}^{N-1}
\end{array}\right]\left[\begin{array}{c}
B_{1} \\
B_{2} \\
\vdots \\
B_{n}
\end{array}\right]=\left[\begin{array}{c}
y_{0} \\
y_{1} \\
\vdots \\
y_{N-1}
\end{array}\right]
$$

The $N \times n$ matrix in (2.9) is a Vandermonde matrix.

Up to now, it has been assumed that the order of the signal $n$ is known and that this is the order used in the LP and Vandermonde problems. This is not always the case. In many cases $n$ is unknown, and even when it is known, one often chooses a larger order for the LP and Vandermonde problems for numerical robustness. To simplify explanation in the remainder of this document, we define $n_{L, P}$ to be the order used in the LP problem, $n_{V}$ to be the order used in the Vandermonde problem, and $n$ will continue to be the order of the final model.

\subsection{Literature Review}

Prony analysis was first introduced by Baron de Prony (1795) 200 years ago. Because of lack of modem computing capability, significant applications were not explored until Hildebrand (1956) proposed least-squares solutions. Since the proposition by Hildebrand, several papers have been published on solving Prony analysis types of problems. Probably the most complete and modern discussion of Prony analysis is contained in Scharf (1991). The literature review here is focussed on solution techniques and extensions of Prony analysis. Papers discussing the application of Prony analysis are not reviewed here.

The original Prony method proposed that $N=2 n, n_{V}=n_{L P}=n$, and the LP forward-direction covariance formulation be used. The LP and Vandermonde systems are then directly solved using matrix inversion; the Vandermonde system is made square by using only the first $n$ data points. Even with modern computers, this formulation is extremely numerically sensitive (Scharf 1991). To avoid these numerical problems, Hildebrand (1956) proposed to allow $N>2 n$ and employ least-squares solution techniques. Much of the modern literature on Prony analysis focuses on robustly solving the 
least-squares problem.

Many researchers have contributed to developing modern least-squares Prony analysis. Some have termed the least-squares solution approach "extended Prony analysis" (Marple 1989). The work by Kumaresan and Tufts (Kumaresan and Tufts 1982; Tufts and Kumaresan 1982a, 1982b) is probably best known for pioneering modern development of Prony analysis by applying advanced numerical techniques to solve the lcast-squares problems. Its main thrust is solving the LP problem.

In Tufts and Kumaresan (1982a, 1982b) methods are presented based on singular value decomposition (SVD) for estimating closely spaced frequencies of sinusoidal signals in noise. Although they do not explicitly call this a Prony method, it is equivalent to assuming that all the poles are on the unit circle. Under this constraint, the forward and backward direction LP polynomials are the same. Significant attention is given to the algorithm described in Tufts and Kumaresan (1982b) where the LP problem is written in a combined forward-backward manner and $n_{L P}$ is maximunily chosen. Discussions of the properties of this method are contained in Rao (1986a, 1986b).

Kumaresan and Tufts (1982) expand their methods to estimate parameters of damped sinusoids in a least-squares sense as proposed by Hildebrand (1956). Again they address the problem of solving for the LP coefficients. The LP problem is written in the backward direction, as opposed to the forward proposed in Hildebrand (1956), using the covariance form, and the SVD of the LP matrix is computed. The smaller singular values are "ignored" (their inverse are identically set to zero) in computing the least-squares solution with the SVD matrices. As discussed in Tufts and Kumaresan (1982a), this is the best lower rank approximation to a given matrix (in the Frobenius norm sense). The order of the prediction (i.e:, $n_{L P}$ ) is assumed to be a fraction of the number of data samples (approximately $N / 3$ to $N / 2$ ), which facilitates the robustness of the SVD. By using the backward direction of the LP method, the true signal modes occur outside the unit disk while noise terms are inside the disk.

Using the SVD method to solve over-determined systems of equations has become a highly recommended method (Press et al. 1990). This is due primarily to its robust numerical properties and ease of implementation.

A modification to the procedure used in Kumaresan and Tufts (1982) for estimating the LP coefficients is proposed by Porat and Friedlander (1986). The LP problem is solved both in the forward and backward directions. When the backward direction terms are reflected about the unit circle, the signal poles will reflect onto those from the forward direction analysis while the noise terms will move away from each other. The identified terms are then averaged. The authors claim this algorithm can be used to identify both damped and undamped modes.

Although the methods in Steiglitz and McBride (1965) and Steiglitz (1977) are not strictly Prony analysis methods, they are used to identify impulse response models. Three methods are compared in Steiglitz (1977). The LP problem is solved similar to that proposed by Hildebrand (1956), and an iterative correction algorithm is used to improve on the LP estimation.

Barone et al. (1987) propose using the Burg algorithm to solve the LP problem in covariance form. They term this the Prony-Burg method. The Burg analysis is an iterative one where $2 n_{L P} L P$ polynomials are calculated. It has proven successful with Maximum Entropy Spectral Estimation (MESA) (Kay and Marple 1981). The Akaike method (1969) is used to determine the actual LP 
model order $(n)$ and thus the proper LP polynomial. Applying the Akaike method to this problem results in the final prediction error (FPE) being minimized at the proper model order $(n)$, where

$$
F P E=\frac{N+n+1}{N-n-1} \sigma^{2}
$$

with $\sigma^{2}$ being the squared error between model and data. Barone et al. (1987) recommend that eigenanalysis be used for calculating the roots of the LP polynomial and SVD be used for solving the Vandermonde system.

Four different methods for solving the over-determined covariance LP system are compared in Barone (1988); these methods are: 1) a least-squares covariance algorithm using an orthogonalization method based on Givens rotations (Kay and Marple 1981); 2) the covariance SVD method proposed in Tufts and Kumaresan (1982a); 3) the Burg algorithm (Kay and Marple 1981); and 4) the Marple algorithm (Marple 1980). The methods are compared by applying the four to an example problem consisting of sinusoids in noise. It is concluded that for this example, the Marple and Burg algorithms perform best.

Additional methods for solving the LP problem are proposed (Minami et al. 1986; Cruz 1986). In Minami et al. (1986) the LP problem is solved using eigenvalue decomposition of the covariance matrix. By discarding eigenvalue and eigenvector terms that are too "small," the optimal model order $(n)$ is determined during the fit. Cruz (1986) proposes that the quotient-difference (QD) algorithm of Rutishouser (1957) be used to solve the LP problem. Using this algorithm allows the system poles to be directly identified, removing the need for root finding or eigenanalysis. No comparisons are made in Minami et al. (1986) or Cruz (1986) with more established methods such as SVD.

Demeure and Scharf (1990) describe a "fast" recursive orthogonalizing (QR) method for decomposing the Toeplitz matrix in the LP problem. Cnce the decomposition is complete, the LP problem is easily solved. For high-order problems, the algorithm offers significant speed-up when compared to standard Cholesky methods. The technique is also described in Scharf (1991).

The problem of choosing the model order $(n)$ and the signal components is addressed by Kumaresan, Tufts, and Scharf (1984). A least-squares solver (such as SVD) is used to estimate the LP parameters with $n_{L P}$ being near $N / 3$ to $N / 2$. Then $n_{V}$ is varied from 1 to $n_{L P}$, and the best model for each $n_{V}$ is calculated by searching all the $z_{i}$ 's for the $n_{V}$ subset that best represents the signal. Two search methods are possible: 1) try every possible combination of mode terms; or 2) search for the terms one at a time using the search technique described in Hocking and Leslie (1967). The second is less accurate than the first, but it requires much less calculation time. The true model is selected as the one that maintains a minimum error between the signal data and model output with minimum model order. The optimal order is subjective and difficult to algorithmize.

A similar model-order selection technique that employs a model reduction method has been 
developed at PNL. ${ }^{(a)}$ With this method, $n_{L P}=n_{V}$ are assumed large $(N / 3$ to $N / 2)$, and the LP and Vandermonde problems are solved for order $n_{L P}$. The model order is then reduced by selecting the subset of residue and pole terms that minimize an Akaike information criterion with the associated error calculated in both the time and frequency domains. The method is much less time consuming than that in Kumaresan, Tufts, and Scharf (1984), as only one Vandermonde problem must be solved.

In the previously mentioned literature, little attention is given to factoring the LP polynomial to obtain signal poles. Basically, two methods are possible: eigenanalysis and polynomial factoring. Only a few papers address polynomial factoring, and no published papers were obtained that discussed different eigenanaiysis routines.

Three methods of calculating the roots of a real-coefficient polynomial are compared in Rautenberg et al. (1988). Special attention is devoted to polynomials such as those found within Prony analysis. The three algorithms compared are; 1) the Madsen (1973) method; 2) the Muller (1956) iterative algorithm; and 3) the three-stage method by Jenkins and Traub (1970). Comparisons are based on accuracy, convergence rate, and stability. The Jenkins and Traub method performs best in most cases. For example, using double-precision arithmetic, the Jenkins and Traub method can accurately calculate the roots of a 60th order polynomial compared to 20 and 30 for the other two.

An algorithm for concurrently calculating the roots of a polynomial is proposed in Hoxha, Lambert, and Noailles (1988). Such an algorithm could be implemented on a parallel processing computer to accelerate computation time of Prony analysis. Examples are given for various versions of th: algorithm, but no comparison is made with more conventional algorithms.

Demeure (1989) uses a fast QR factorization of the Vandermonde matrix to solve for the residues. Versions described include algorithms for complex conjugate poles and for poles with multiplicity greater than or equal to one.

Data pre-conditioning is proposed in Friddell et al. (1989) for improving the performance of Prony analysis under low signal-to-noise ratio (SNR). First, pre-filtering is examined with only limited success. Then a method that utilizes the fast Fourier transform (FFT) in conjunction with filtering to "zoom-in" on a portion of the signal spectrum is proposed. Basically, an FFT is performed on the signal, then all parts outside the regions of interest in the frequency domain are removed. An inverse Fourier transformation (IFT) is then used to obtain a clean time-domain signal.

A recursive form of Prony analysis is proposed in Lambert and Castanie (1988). With this algorithm pole and residue estimates are updated with each sample. Simulations demonstrate that the algorithm is able to track step and drift changes in the parameters of a sinusoid in noise.

McWhorter and Scharf (1991) examine the error bounds of Prony analysis (and other general modal analysis methods). They compute and examine the Cramer-Rao (CR) bounds for many of the parametric descriptions of a mode including AR and MA coefficients, poles and residues, and poles and zeros. All are related through transformations. The CR bounds provide a theoretical lower limit

(a) D. J. Trudnowski. 1993. "Order Reduction of Large Scale Linear Oscillatory System Models." To be presented at the 1993 IEEE Power Engineering Society Winter Meeting, Columbus, Ohio, February 1993. 
for estimated parameters. An interesting point made in McWhorter and Scharf (1991) is that the CR bound is valid for any unbiased estimator. That is, the theoretical lower limit of an estimator's error is not dependent on that estimator algorithm, but is dependent on the information contained in the sampled data. The Fisher Information Matrix used in determining the CR bounds is also used to generate the smallest achievable "concentration ellipse" for a parameter set. These concentration ellipses define the smallest possible confidence ellipse within which the true parameters may lie with a given probability. Many examples are used to demonstrate the usefulness and properties of these error bounds.

In order to use the forms of Prony analysis discussed up to this point for system identification, the excitation to the system must be an impulse function. In reality, it is often extremely difficult to apply such an excitation to an actual system. Using Prony type analysis for system identification has been investigated for many different input forms.

Basically, the system identification problem is addressed from two perspectives. With the first, an input is applied to the system and Prony analysis is used to analyze the output after the input is turned off. Then the transfer function terms are obtained through algebraic functions involving the Prony fit terms. The second perspective involves modifying the Vandermonde problem to directly identify system transfer function terms. The advantage of this case is that all of the output data is used to calculate transfer function residues as opposed to just the data when the input is off. Using all the data in the LP problem is more difficult and can be done only if the input stays on for a significant number of samples (at least double the assumed system order).

The results in Poggio et al. (1978) show the equations required for identifying a strictly proper transfer function using Prony analysis when the input is

$$
u(t)=e^{\tau_{1} t}-e^{\tau_{2} t}
$$

where $\tau_{1}$ and $\tau_{2}$ are real time-constants. With this formulation, it is assumed that the system does not have poles at $-\tau_{1}$ or $-\tau_{2}$. In Hauer (1991) the input is allowed to be a square wave pulse, but the system is assumed not to have a pole at zero. The allowed input is expanded in Trudnowski et al. (1991) to be a finite luration of pulses with the same time constant, which includes a pulse train. But again, the system $\mathrm{ml}:$ be trictly proper and cannot have a pole corresponding to the input's time constant.

The work in Pierre, Trudnowski, and Hauer (1992) and Pierre et al. (1992) as well as recent work at $\mathrm{PNL}^{(\mathrm{a})}$ allow the system to be proper and to have non-zero initial conditions. Also, the system is allowed to have poles corresponding the to input's time constants. The input is expanded to be a finite-dimensioned Laplace-transformable function, which includes pulse trains, step functions, sinusoids, and a linear combination of these. The general versions proposed in Pierre et al. (1992) and in the recent PNL work $^{(a)}$ uses all the system data to calculate transfer function residues and feedforward terms.

(a) D. J. Trudnowski, M. K. Donnelly, and J. F. Hauer. 1993. "Advances in the Identification of Transfer Function Models Using Prony Analysis." Submitted for presentation to the 1993 American Control Conference, San Francisco, California, June 1993. 


\subsection{Prony Analysis Codes}

Primarily through BPA, several related Prony analysis computer codes have been developed. All are FORTRAN-based. In this section the content, differences, and status of these codes are summarized. Attention is paid primarily to the options in the core mathematical routines.

It is somewhat difficult to compare these codes in detail, as no previous effort has been given to formally track code updates and modifications. PNL has informally begun to track the versions of the routines that make up these codes by using the Revision Control System (RCS) described in Tichy (1985). The versions discussed here are the latest versions obtained by PNL and are likely the most recent.

The programs described here include SIGPAKZ, IPRONY, IPRONYID, and TRANSIENTS. The first three codes use primarily double-precision arithmetic and are built around the subroutine PRSPAK which performs the actual calculations of the Prony analysis fit. TRANSIENTS is a Prony analysis program developed at the University of Colorado at Boulder (UC) and uses primarily single-precision arithmetic. An early double-precision version of TRANSIENTS called DTRANSIENTS was used as the basis for PRSPAK. The current version of TRANSIENTS has several new options and modifications not included in PRSPAK.

\subsection{SIGPAKZ and IPRONY}

SIGPAKZ was developed at BPA and is a noninteractive program that uses the PRSPAK routine to perform the Prony analysis fit. No formal documentation or manual has been published for SIGPAKZ, but some detailed explanations are contained in Hauer et al. (1990) and Hauer and Taylor (1990) and in examples available with the program. It has several very distinct advantages, including the following: reads many data file formats for the system response data, including many powerindustry-specific formats; allows "sliding window" fits; permits transfer function identification for a single pulse or step input and a proper system with no poles at zero; has several data-conditioning options (e.g., detrending and scaling); and has several output options including files for direct analysis using other BPA programs.

IPRONY is a stripped interactive version of SIGPAKZ. It calls the routine PRSPAK without the many options available in SIGPAKZ. It is very similar to an earlier version of DTRANSIENTS.

\subsection{IPRONYID}

IPRONYID is an interactive transfer function identification program that also uses PRSPAK as the core Prony fitting routine along with other routines developed at PNL. ${ }^{\text {(a) }}$ The system input is allowed to be an impulse function or a finite number of square-wave pulses (including step functions). The transfer function model is allowed to be proper and can have a pole at zero (in the s-plane).

IPRONYID also performs model reduction to separate true system terms from those representing noise

(a) D. J. Trudnowski. 1992. "IPRONYID Version 1.1b: A Computer Program for the Identification of Transfer Functions Using Prony Analysis." Pending PNL report. 
effects.

The version of PRSPAK used in IPRONYID is a variation of that called by SIGPAKZ. Modifications were made so that PRSPAK does not necessarily have to write to a data file during execution in batch mode. Changes were made so that this new version can easily be used with SIGPAKZ and IPRONY. Several tests were conducted to compare the results from SIGPAKZ and IPRONYID. As expected, under the same input conditions, they both give the same results.

\subsection{PRSPAK (Core Prony Subroutine)}

SIGPAKZ, IPRONY, and IPRONYID all use versions of the subroutine PRSPAK which performs the actual Prony analysis. The version of PRSPAK used in SIGPAKZ and IPRONY is PRSPAK2 version 1.1 (the version number has been adopted at PNL for convenience). This version was obtained from BPA in a send-out tape (dated June 1991). Version 1.1.2.1 of PRSPAK2 is contained in Appendix A and is the version used in IPRONYID. Three main functions are performed in PRSPAK: 1) solution of the LP problem; 2) rooting of the LP polynomial; and 3) solving the Vandermonde problem. There are 12 different ways of formulating the LP problem, and 4 possible solution methods for cach formulation. Also, there are 2 solution options for the root finding problem, and 3 different solution methods for the Vandermonde problem. This means that there are 288 possible solution paths available in PRSPAK for solving the Prony analysis problem. As can be seen, PRSPAK calls many other subroutines.

With the LP problem, the user must first choose the linear prediction method from one of the following: correlation; pre-windowed; covariance; and post-windowed options. This determines the form of the Toeplitz matrix as described by Equation (2.8). Then the forward/backward logic must be determined. PRSPAK allows the LP equation to be written in the forward direction as in (2.5), or the backward direction as in (2.6), or a combination of these. In the forward direction, the $a_{i}$ 's are identified; in the backward, the $\bar{a}_{i}$ 's are identified; and in the combination case, both polynomial coefficient sets are identified.

There are four choices for solving the over-determined set of equations representing the LP problem: 1) singular-value decomposition (SVD); 2) standard QR; 3) total least-squares (TLS); and 4) fast QR;. The SVD is performed on the Toeplitz matrix using a standard double-precision LINPACK (Dongarra 1979) routine, and the solution is obtained using the standard SVD method (Press et al. 1990). Also, the QR factorization routine in double-precision LINPACK is used to solve the LP problem using the standard QR (again, see Press et al. [1990] for details). The fast QR method is described in Demeure and Scharf (1990).

The TLS method is slightly different than solving (2.8) in a least-squares sense. Let (2.8) be rewritten in matrix form as

$$
Y A=0
$$

In solving (3.1) using TLS, we assume that an error matrix is associated with $Y$. Let's call this error 
matrix $\delta Y$. Now Equation (3.1) is written as

$$
(Y+\delta Y) A=0
$$

TLS consist of minimizing the Frobenius norm of $\delta Y$, under the constraint that the (3.2) is satisfied. The TLS problem in PRSPAK is solved using singular-value decomposition. The Toeplitz matrix is factored into its singular-value components as

$$
Y=U \Sigma V^{T}
$$

where $U$ and $V$ are the left and right singular-value unitary matrices and $\Sigma$ is a diagonal matrix of singular values (see Dongarra et al. [1979] for details). The solution vector $A$ is then

$$
A=-v_{m}
$$

where $v_{m}$ is the appropriately normalized $m$ th column of matrix $V$, and $m$ corresponds to the location of the minimum singular value in $\Sigma$. Again, the double-precision LINPACK SVD routine is used in PRSPAK to perform the SVD on the Toeplitz matrix.

Only one root finder option is available in PRSPAK, although it can be performed in either a double (DPOLYRT) or quad-precision (QPOLYRT) subroutine. The root finding routine was taken from the "SAMSAN" codes by Dr. John Hauer of BPA. From the notes in the subroutines, it is a version of RPOLY described in Jenkins (1975). But, it is not clear from the code that this is the actual algorithm used in DPOL:YRT and QPOLYRT.

The third calculation performed in PRSPAK is determining the signal residues through the solution of the Vandermonde problem. There are three possible solution methods: 1) standard QR; 2) fast real; and 3) fast complex. As with the LP problem, double precision LINPACK routines are used to solve the standard QR problem. The two fast routines are taken from Demeure (1989).

PRSPAK allows the user to choose the order of the Vandermonde system $\left(n_{V}\right)$ to be less than the order of the LP $\left(n_{L P}\right)$. If $n_{V}$ is less than $n_{L P}$, PRSPAK solves all the possible Vandermonde problems that represent all combinations of $z_{i}$ 's taken $n_{V}$ times. The one solution combination that best fits the data in a squared-error sense is taken as the final solution.

Other functions performed in PRSPAK fall into one of the following categories: interactive input/output; batch output; default parameter selection; miscellaneous calculations; and plotting. The plotting routines compile only under specific platforms and are activated only when PRSPAK is operated in the interactive mode.

\subsection{TRANSIENTS}

TRANSIENTS is an interactive Prony analysis program being developed at UC. The version currently at PNL was the latest version available from UC in spring 1992. As previously mentioned, the current versions of PRSPAK were developed by modifying an older version of TRANSIENTS. Since that time, several modifications have been made to TRANSIENTS and to the several supporting subroutines. 
TRANSIENTS has the same solution options as PRSPAK, although many minor changes have been made to nearly all the subroutines as compared to those employed in PRSPAK. A new major addition to TRANSIENTS includes the solution of composite mode problems. That is, it allows the assumed model to have repeated poles.

\subsection{Computer Platforms}

Because all the Prony analysis codes are FORTRAN-based, it is possible to port them to different computer platforms if an adequate FORTRAN compiler is available. In this section, PNL's experience with different platforms is summarized.

SIGPAKZ and IPRONY, as delivered from BPA, execute under a VAX-VMS operating system. At PNL this code was ported to a SUN Sparc 2 workstation operating under the UNIX system. The SUN version 1.4 FORTRAN compiler was used to compile and link the programs on the Sparc 2. This porting required a number of modifications. Summaries of these modifications are described here.

Modifications to SIGPAKZ include the following:

1. Changing file names in INCLUDE statements to lower case because UNIX differentiates between capital and lower-case letters.

2. Adding parentheses to the argumencs of PARAMETER statements as required by the SUN compiler.

3. Changing the default $\mathrm{I} / \mathrm{O}$ unit numbers from 5 and 6 to 2 and 3 . SUN reserves 5 and 6 .

4. Modifying con non files LUNITS.CMN and PPLOT.CMN to remove redundancies in COMMON and DATA statements.

5. Modifying OPEN statements in LUSET.FOR to use SUN I/O options.

6. Using the SUN version of the random number generator in functions DALEAT and ALEAT in file MATH.FOR.

7. Adding a new FORMAT statement in RDSIG for reading character data. With the SUN compiler the FORMAT must match the declared dimension of a character string. As more read options are used with the SUN version of SIGPAKZ, more changes of this type will likely have to be made.

8. Routines and functions in files NEWGRAPH.FOR, PPLOT.FOR, VERSA2.FOR, and VPLOT.FOR that make calls to PLOT10/LIB and VTRANOP.OBJ were disabled with comment markers. This was done because PLOT10/LIB and VTRAN.OBJ are not available for porting; therefore, they are not included in the link list. Also, the routine CPUTIME.FOR was disabled because it makes specific VMS calls; if so desired, these calls could be replaced with SUN-specific calls. 
With these changes SIGPAKZ successfully compiles and links under the SUN system. Several test cases were run both on the VMS version and the SUN version of SIGPAKZ. Results matched between the two versions out to the 9th or 10th decimal place with all the tests. The slight difference between the two is likely due to the different interpretations of double precision between VAX and SUN platforms. SUN uses the IEEE Standard for Binary Floating-Point arithmetic. For a 64 bit number, 11 bits are devoted to the exponent which leaves 53 significant bits. VAX in D_floating format reserves 8 bits for the exponent leaving 56 significant bits.

With similar differences, IPRONYID also compiles under both the VAX-VMS and SUN platforms. Also, IPRONYID was compiled under a DOS operating system using a Lahey ${ }^{(a)}$ compiler. With this DOS version, the double precision root finder must be used as the Lahey compiler does not support quad-precision. Only a very few/ other minor changes were made to the SUN version of IPRONYID to make it compile under DOS.

TP.ANSIENTS is being developed under a Hewlett Packard (HP) platform using several HP specific plotting options. These plotting options are scattered throughout most the subroutines. Therefore, the majority of modifications required to port TRANSIENTS to a SUN platform involved disabling the appropriate plotting options. The only other required modification involved changing the calling to a HP specific random number generator.

(a) Developed by Lahey, Inc. 


\subsection{Example Systems and MATLAB Interfaces}

To effectively evaluate the characteristics of Prony analysis, response data from linear systems under controlled conditions were required (including noisy conditions). The purpose of this section is to describe the test systems, synthetic noise characteristics, and interfacing with the commercial software package MATLAB, which was developed by the Mathworks, Inc.

\subsection{Interfacing with MATLAB}

Much of the data processing for conducting SIGPAKZ and IPRONYID runs was done using the software package MATLAB and its associated Control System Toolbox. This includes the development of test systems. Details on interfacing SIGPAKZ and IPRONYID files with MATLAB are described here as are several MATLAB m-files for performing specific tasks.

MATLAB (standing for matrix laboratory) is a multi-purpose interactive program that allows a user to easily access several advanced numerical and matrix analysis methods. When used with the Control System Toolbox, it can solve many advanced analysis problems related to linear systems. A few of the functions include time-domain simulation, frequency domain analysis, conversion between model formulations (e.g., state-space to ratio of zeros and poles), and root locus analysis. MATLAB allows the user to write custom functions using $m$-files.

Interfacing MATLAB with SIGPAKZ and IPRONYID is done using a combination of FORTRAN programs and $m$-files. To save a data set for processing by SIGPAKZ, the data are saved in MATLAB as a straight ASCII file; this ASCII file is then converted to a readable SIGPAKZ file using the FORTRAN program CONVERT2SWX. To import the results from a SIGPAKZ execution, the output model is extracted from the SIGPAKZ output file and then loaded into MATLAB using the load command. The $m$-file SIGZ2MAT.M is then used to convert the data to residue/pole model in MATLAB. IPRONYID outputs are extracted by running the FORTRAN program IPR2MAT and then loading the appropriate file into MATLAB and running the m-file IPR2MAT.M.

Several other $\mathrm{m}$-files have been developed for the work presented in this report. Some of the less trivial $\mathrm{m}$-files include

- PAR2SS.M: Convert a model in parallel form to block-diagonal state-space form. SS2PAR.M performs the vise-versa.

- ODEABCD.M and ODEABCD2.M: Perform a time simulation of a linear model in statespace form (errors have been encountered with the similar MATLAB function LSIM).

- ORDER.M: Performs model reduction.

- PARRID.M: Given a system input, output, and Prony fit to the output, calculate the transfer function residues.

- THRUPUT.M: Given the results from PARRID.M, calculate a least-squares fit to the throughput term (or feed-forward term). 
- $\quad$ REFLECT.M: Reflect system poles and zeros about the $j \omega$ axis.

- IMPULSEPAR.M and BODEPAR.M: Calculates impulse and frequency responses for systems in parallel form (similar to MATLAB functions IMPULSE and BODE for statespace forms).

\subsection{Test Systems}

Characteristics of Prony analysis were investigated using variations of three linear systems. The first system is a 9th-order, 4-oscillator system; the second is a 12th-order, 5-oscillator system; and the third is a 28th-order, 14-oscillator system. The second and third systems were obtained from BPA.

\subsubsection{Test System I}

Test system 1 is represented by the parameters in Table 4.1, where " \pm " is used to iepresent complex conjugate pairs. Using the notation in Table 4.1, the transfer function for this test system can be written as

$$
G(s)=\sum_{i=1}^{n} \frac{\operatorname{Residue}(i)}{s-\operatorname{Pole}(i)}
$$

or as

$$
G(s)=\frac{\operatorname{Gain} \prod_{i=1}^{n}(s-Z e r o(i))}{\prod_{i=1}^{n}(s-\text { Pole }(i))}
$$

where $n$ is the number of poles and $m$ is the number of zeros. Figure 4.1 shows the impulse response of test system 1, and Figures 4.2 and 4.3 show the frequency response. This system is characterized by two dominant modes at 0.35 and $0.45 \mathrm{hz}$, and two weaker, more lightly damped modes at 0.8 and $1.0 \mathrm{hz}$. After $1.6 \mathrm{hz}$, the frequency response of the system tends to roll off because of the real pole.

Table 4.1. Test system 1 .

\begin{tabular}{||l|l|l|l|}
\hline \multicolumn{1}{|c|}{ Poles } & \multicolumn{1}{|c|}{ Residues } & \multicolumn{1}{c|}{ Zeros } & \multicolumn{1}{c|}{ Gain } \\
\hline$-1.60(2 \pi)$ & 44.062 & $-0.80 \pm j 0.55(2 \pi)$ & $9.6 \pi$ \\
$-0.50 \pm j 0.35(2 \pi)$ & $57.46 \angle \mp 74.47^{\circ}$ & $-0.15 \pm j 0.65(2 \pi)$ & \\
$-0.25 \pm j 0.45(2 \pi)$ & $25.07 \angle \pm 137.87^{\circ}$ & $-0.30 \pm j 0.90(2 \pi)$ & \\
$-0.10 \pm j 0.80(2 \pi)$ & $3.00 \angle \mp 109.55^{\circ}$ & $-0.60 \pm j 1.50(2 \pi)$ & \\
$-0.05 \pm j 1.0(2 \pi)$ & $3.425 \angle \mp 143.10^{\circ}$ & & \\
\hline
\end{tabular}




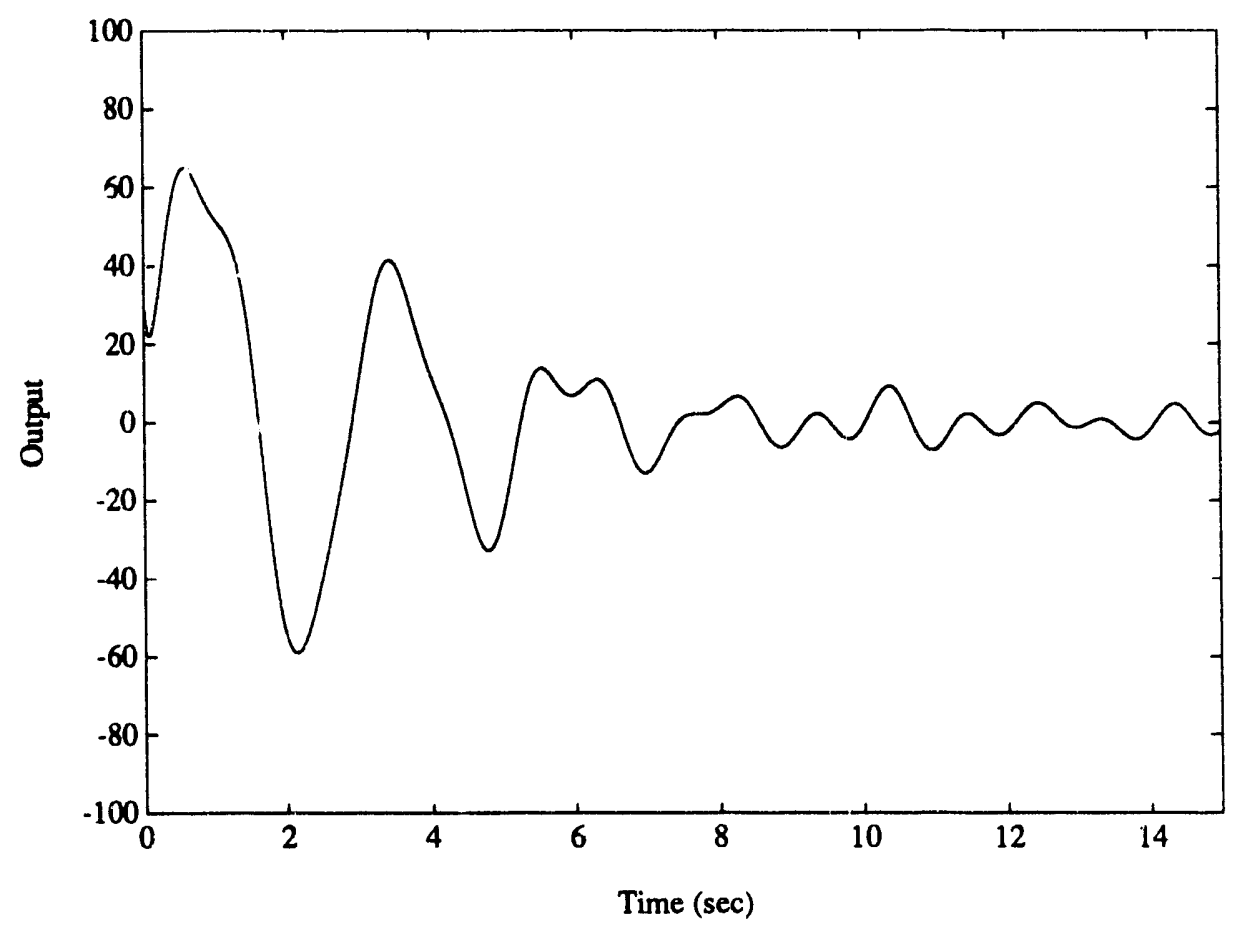

Figure 4.1. Impulse response of test system 1.

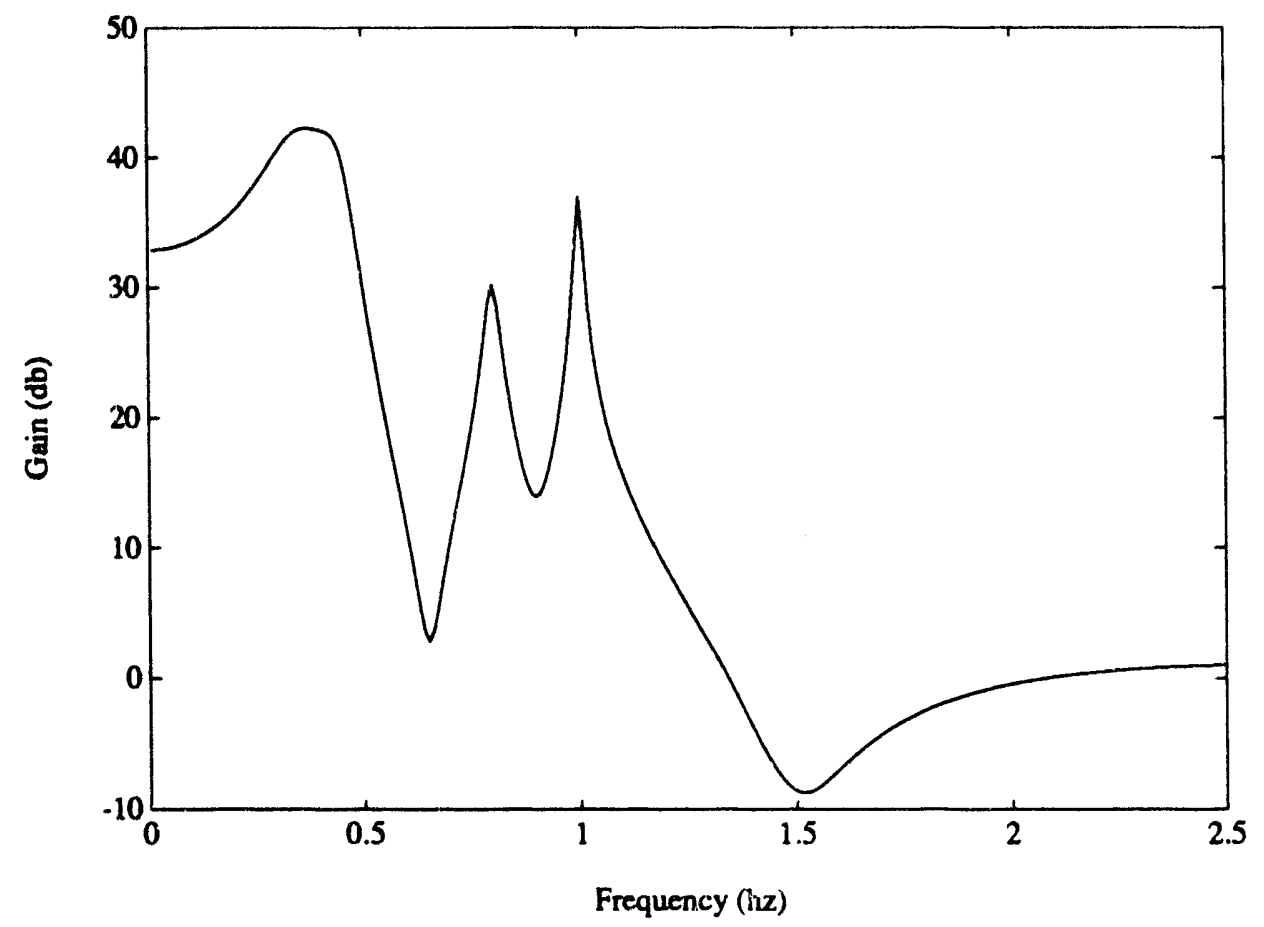

Figure 4.2. Frequency response of test system 1. 


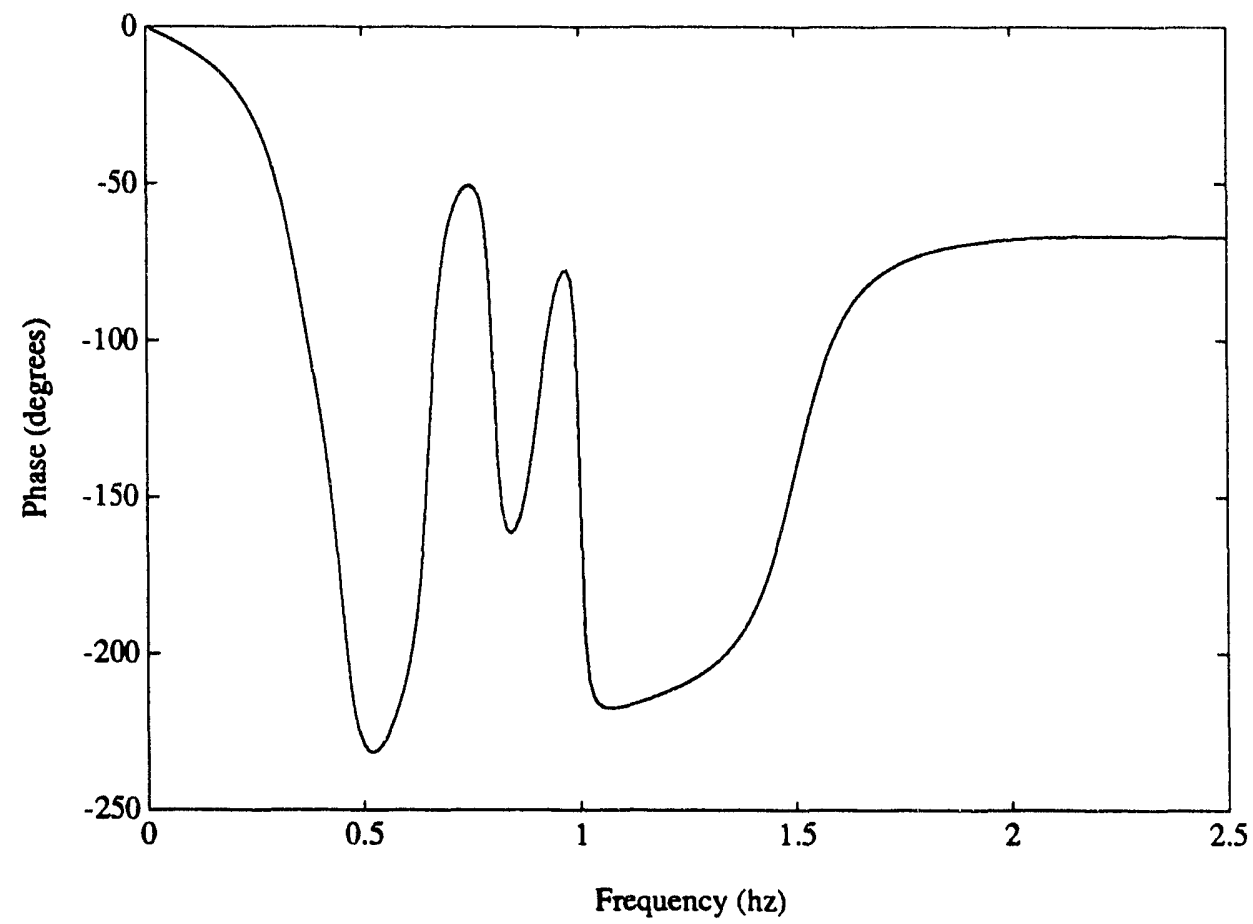

Figure 4.3. Frequency response of test system 1.

\subsubsection{Test System 2}

Test system 2 is shown in Table 4.2 and Figures 4.4 through 4.6 . The 0.38 -hz mode primarily dominates the response.

Table 4.2. Test system 2 .

\begin{tabular}{|l|l|l|l||}
\hline \multicolumn{1}{|c|}{ Poles } & \multicolumn{1}{|c|}{ Residues $\left(\times 10^{-3}\right)$} & \multicolumn{1}{|c|}{ Zeros } & \multicolumn{1}{c|}{ Gain } \\
\hline$-0.7166(2 \pi)$ & -0.003929 & 0 & -6.964 \\
$-2.644(2 \pi)$ & 0.02893 & $-0.4547(2 p i)$ & \\
$-0.08182 \pm j 0.1087(2 \pi)$ & $4.0013 \angle \mp 163.0^{\circ}$ & $-0.7083(2 p i)$ & \\
$-0.04014 \pm j 0.3802(2 \pi)$ & $3.398 \angle \pm 29.26^{\circ}$ & $-2.653(2 p i)$ & \\
$-3.879 \pm j 1.178(2 \pi)$ & $5.262 \angle \pm 128.67^{\circ}$ & $-0.1074 \pm j 0.5825(2 \pi)$ & \\
$-0.2187 \pm j 1.398(2 \pi)$ & $4.381 \angle \mp 26.61^{\circ}$ & $-0.1691 \pm j 2.008(2 \pi)$ & \\
$-0.2591 \pm j 2.160(2 \pi)$ & $0.3872 \angle \mp 56.48^{\circ}$ & & \\
\hline
\end{tabular}




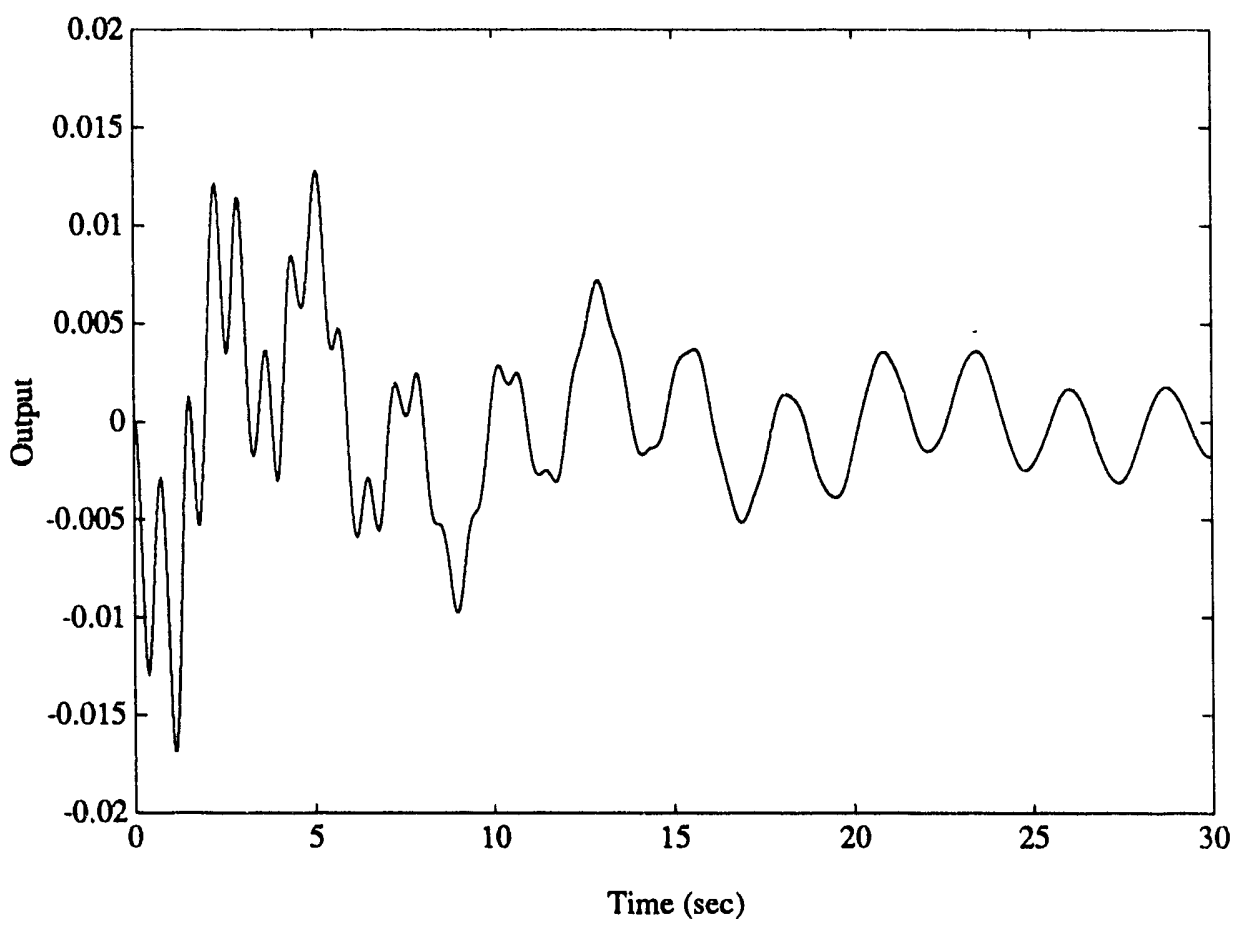

Figure 4.4. Impulse response of test system 2.

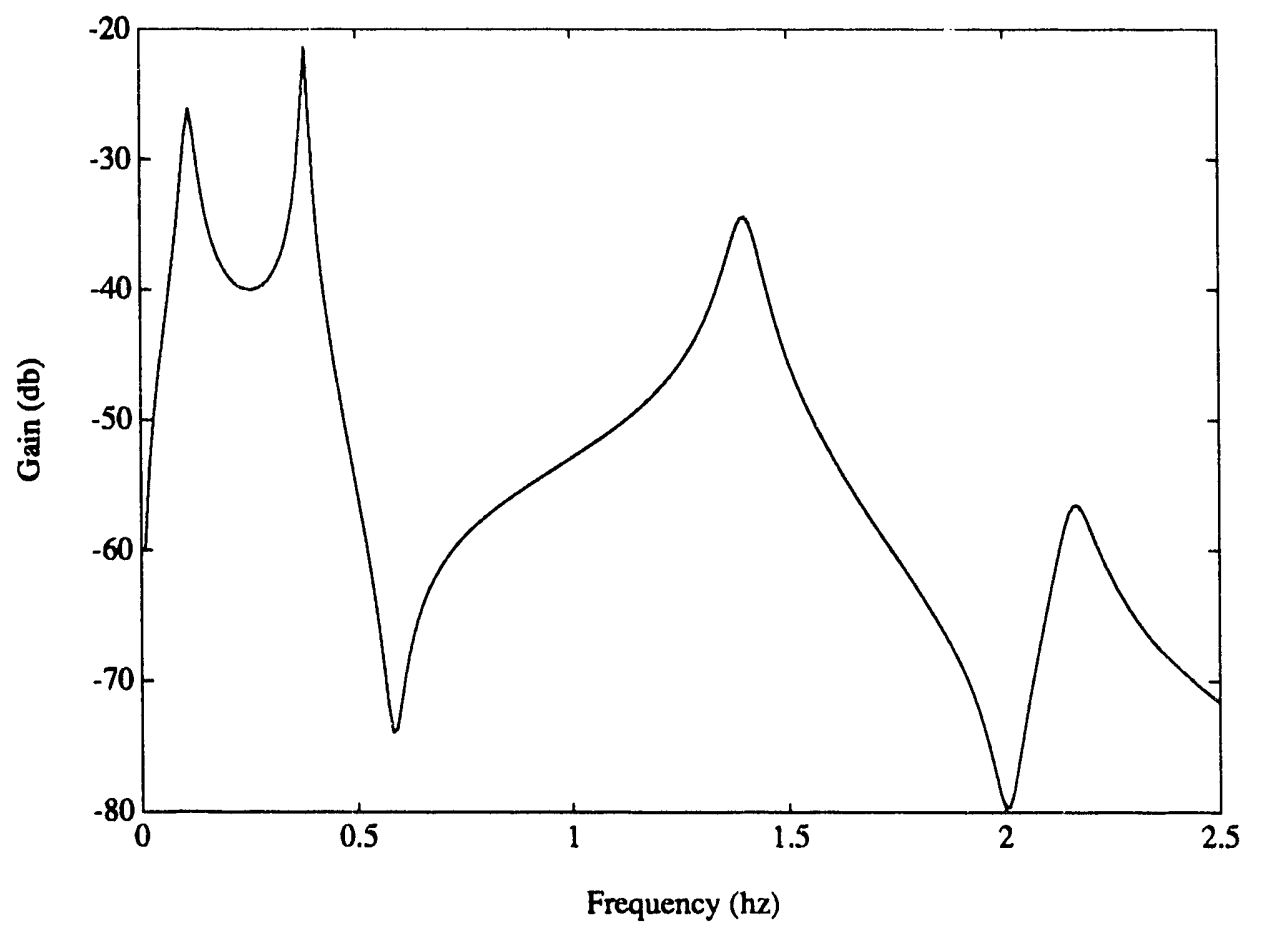

Figure 4.5. Frequency response of test system 2 . 


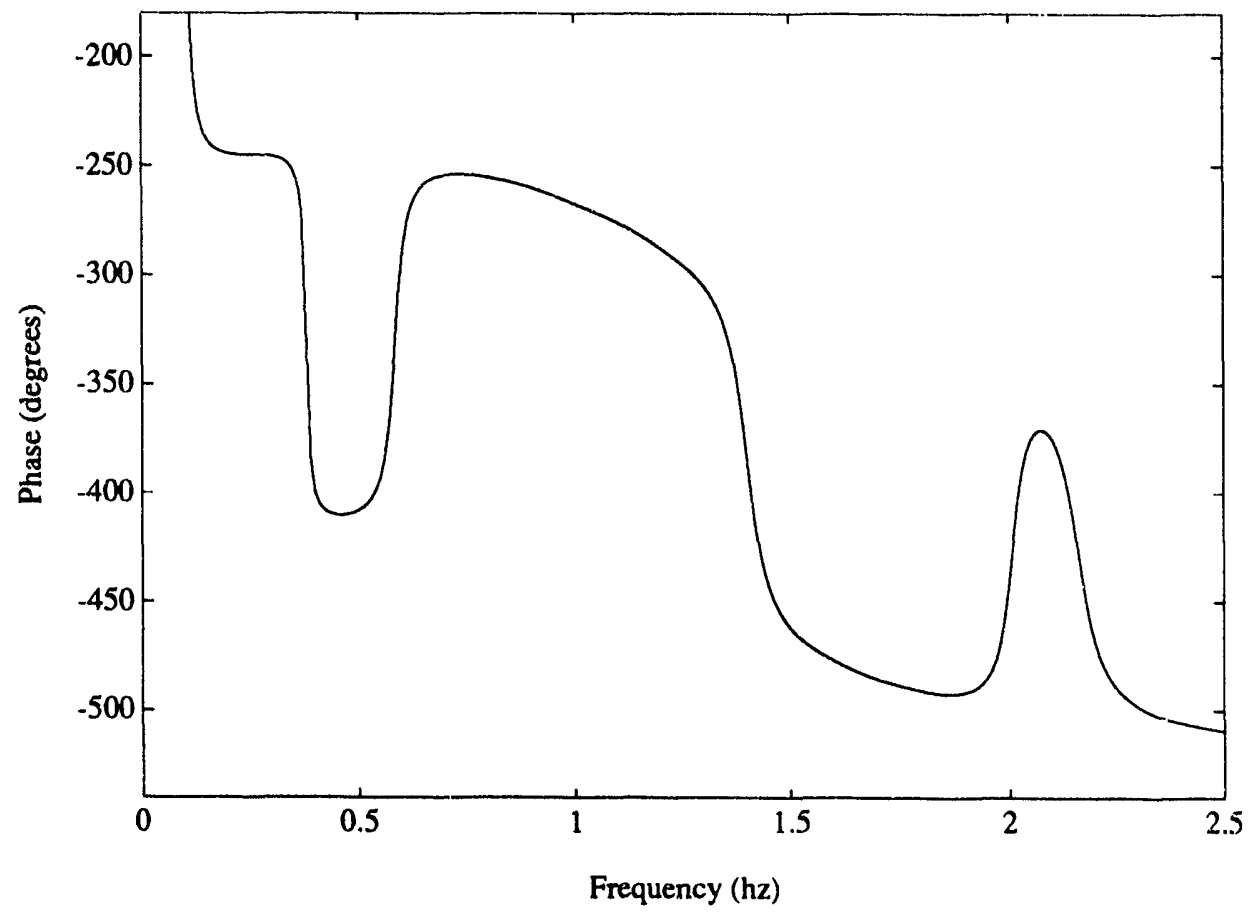

Figure 4.6. Frequency response of test system 2.

\subsubsection{Test System 3}

Test system 3 is represented in Table 4.3 and Figures 4.7 through 4.9.

Table 4.3. Test system 3 .

\begin{tabular}{||c|l|l|l||}
\hline \multicolumn{1}{|c|}{ Poles } & \multicolumn{1}{|c|}{ Residues $\left(\times 10^{-3}\right)$} & \multicolumn{1}{|c|}{ Zeros } & \multicolumn{1}{c|}{ Gain } \\
\hline$-0.9088 \pm j 1.606(2 \pi)$ & $12.21 \angle \mp 61.03^{\circ}$ & -0.03157 & 0.003876 \\
$-0.2414 \pm j 1.502(2 \pi)$ & $3.370 \angle \pm 167.1^{\circ}$ & -2.664 & \\
$-0.1669 \pm j 1.292(2 \pi)$ & $2.035 \angle \mp 28.9^{\circ}$ & $-2.475 \pm j 1.364(2 \pi)$ & \\
$-0.1112 \pm j 1.240(2 \pi)$ & $2.110 \angle \mp 86.2^{\circ}$ & $-2.475 \pm j 1.364(2 \pi)$ & \\
$-0.3525 \pm j 1.151(2 \pi)$ & $4.579 \angle \mp 21.8^{\circ}$ & $-0.1809 \pm j 1.270(2 \pi)$ & \\
$-0.3978 \pm j 1.043(2 \pi)$ & $3.806 \angle \mp 84.7^{\circ}$ & $-0.2882 \pm j 1.245(2 \pi)$ & \\
$-0.1615 \pm j 0.7427(2 \pi)$ & $17.66 \angle \pm 175.0^{\circ}$ & $-0.35144 \pm j 1.092(2 \pi)$ & \\
$-0.5387 \pm j 0.7275(2 \pi)$ & $26.86 \angle \mp 53.60^{\circ}$ & $-1.555 \pm j 0.9946(2 \pi)$ & \\
$-0.1429 \pm j 0.5975(2 \pi)$ & $13.97 \angle \pm 76.96^{\circ}$ & $-3.722 \pm j 0.6327(2 \pi)$ & \\
$-0.2756 \pm j 0.5358(2 \pi)$ & $14.36 \angle \pm 0.05293^{\circ}$ & $-0.03767 \pm j 0.7804(2 \pi)$ & \\
$-0.3198 \pm j 0.4609(2 \pi)$ & $8.825 \angle \mp 119.2^{\circ}$ & $-2.186 \pm j 0.3209(2 \pi)$ & \\
$-0.3746 \pm j 0.3045(2 \pi)$ & $12.08 \angle \mp 4.998^{\circ}$ & $-1.356 \pm j 0.4281(2 \pi)$ & \\
$-0.2001 \pm j 0.0913(2 \pi)$ & $5.010 \angle \pm 116.6^{\circ}$ & $-0.04561 \pm j 0.5726(2 \pi)$ & \\
$-0.1507 \pm j 0.0367(2 \pi)$ & $7.226 \angle \pm 30.12^{\circ}$ & $-0.08628 \pm j 0.4820(2 \pi)$ & \\
& & $-0.07624 \pm j 0.08101(2 \pi)$ & \\
\hline
\end{tabular}




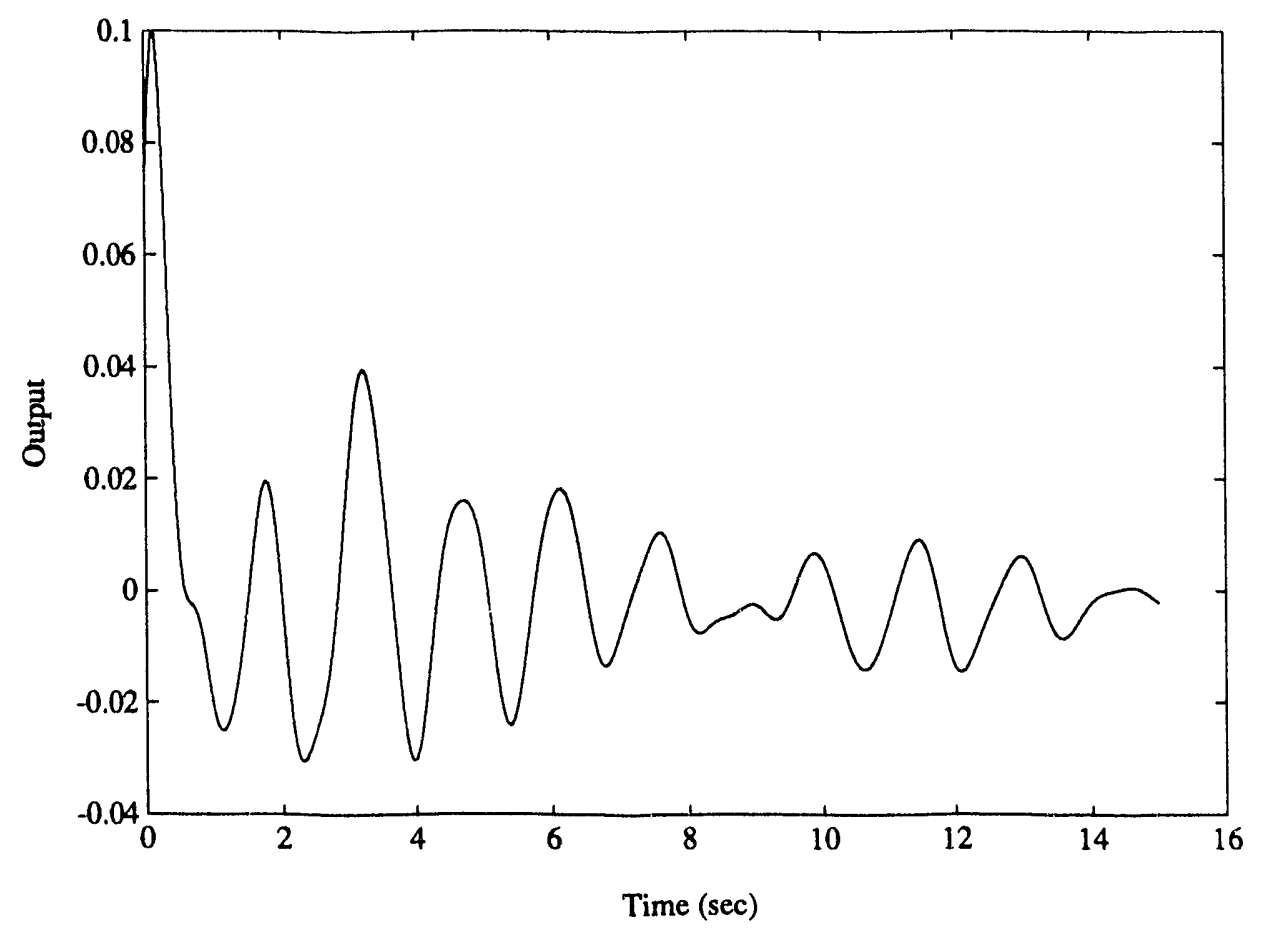

Figure 4.7. Impulse response of test system 3.

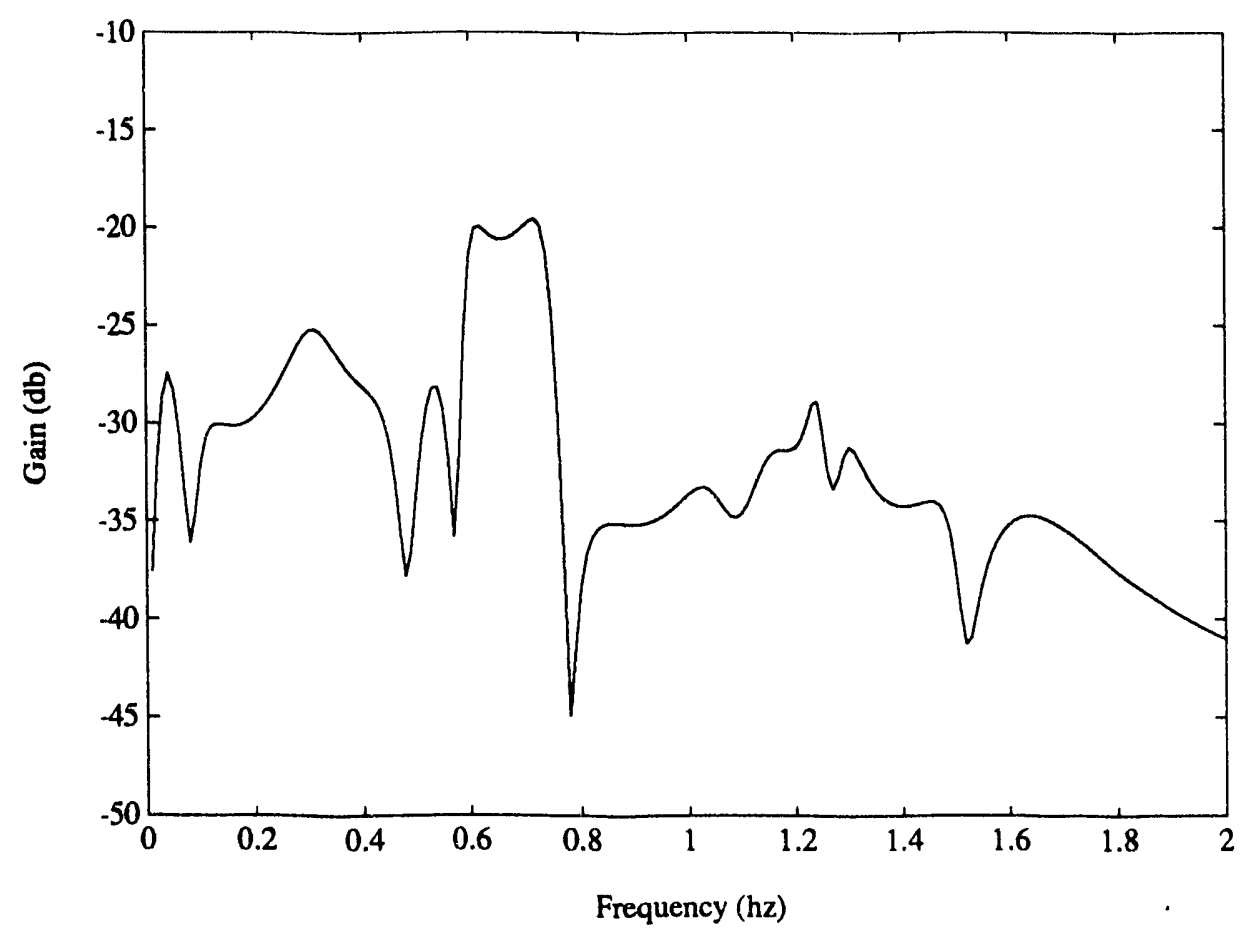

Figure 4.8. Frequency response of test system 3. 


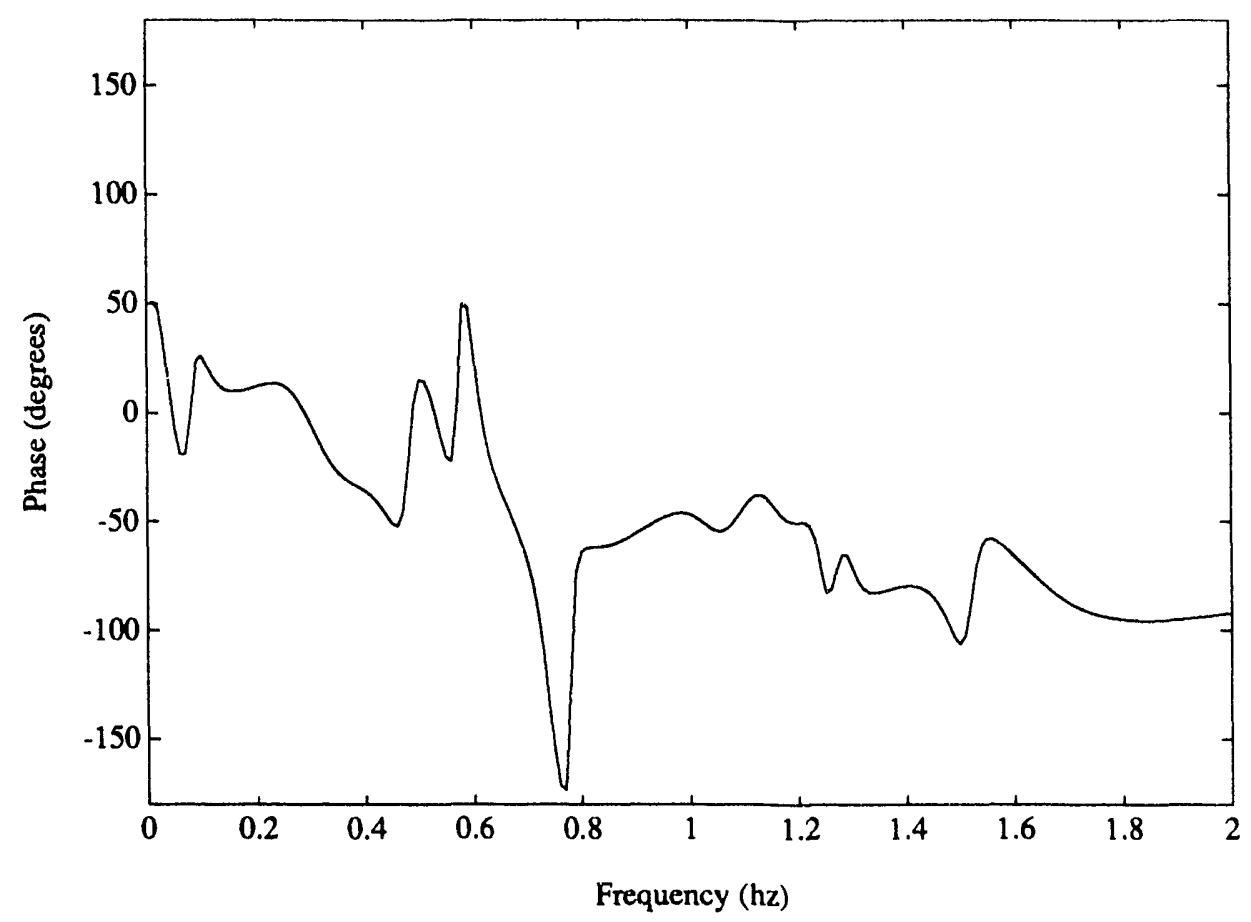

Figure 4.9. Frequency response of test system 3.

\subsection{Noise Models}

To study the ability of Prony analysis to identify accurate models under noisy conditions, several synthetic noise files were constructed. Four noise models, each with its own coloration, are discussed here: 1) white noise; 2) $1 / f$ noise; 3$) 1 / f^{2}$ noise; and 4) sharply colored noise. White noise has a flat power spectrum over the frequency range of interest. The synthetic white noise signals were generated using the stationary Gaussian noise generator in MATLAB.

The power spectrum for $1 / f$ noise rolls off a rate of $1 / f$ where $f$ is the frequency in the range of interest. For the models investigated here, the frequency range of interest is 0.1 to $2.0 \mathrm{hz}$. For this range, $1 / f$ noise is obtained by passing white noise through the nonlinear filter

$$
G_{1 / f}(s)=\frac{(2 \pi 0.1)^{1 / 2}}{s^{1 / 2}+(2 \pi 0.1)^{1 / 2}}
$$

Because (4.3) is not a linear filter, data are passed through it in the frequency domain. The frequency response of Equation (4.3) is calculated and multiplied by the discrete fourier transform of the input time-domain signal. The inverse fourier transformation is then used to obtain the output signal.

Likewise, $1 / f^{2}$ noise has a power spectrum that rolls off at a rate of $1 / f^{2}$ for the range of interest. This noise is generated by passing white noise through the filter described by Equation (4.4). Figure 4.10 shows the frequency response of Equations (4.3) and (4.4). Note that the $1 / f$ noise rolls off at 10 $\mathrm{db} /$ decade and the $1 / f^{2}$ rolls off at $20 \mathrm{db} /$ decade over the frequency range of interest. 


$$
G_{1 / f^{2}}(s)=\frac{2 \pi 0.1}{s+2 \pi 0.1}
$$

White and $1 / f$ noise have very unique characteristics in that their power spectrum decreases linearly with frequency. If audible noise with this characteristic is recorded and played back at a different speed, it will sound the same. These types of noises are called "scaling noises" West (1990). For this reason, and others discussed in (West 1990), $1 / f$ noise is often called fractal noise.

Colored noise is generated by passing white noise through a sharply tuned filter. For the examples discussed later in this report, the coloring filter consists of a 10th-order Chebyshev band-pass filter with comers at 0.4 and $0.7 \mathrm{hz}$. Figure 4.11 shows the frequency response of this filter.

Figures 4.12 through 4.14 show the discrete fourier transformations of the noise sequences used in the examples in Section 5. Two methods of measuring the noise level are employed in later examples. One is the covariance of the noise sequence, and the other is the signal-to-noise ratio (SNR). The covariance is absolute measurement that is a function of the noise only. The SNR is defined here as

$$
S N R=10 \log _{10}\left(\frac{\operatorname{cov}(y(t))}{\operatorname{cov}(n(t))}\right)
$$

where cov is the covariance, $y(t)$ is the deterministic signal, and $n(t)$ is the noise.

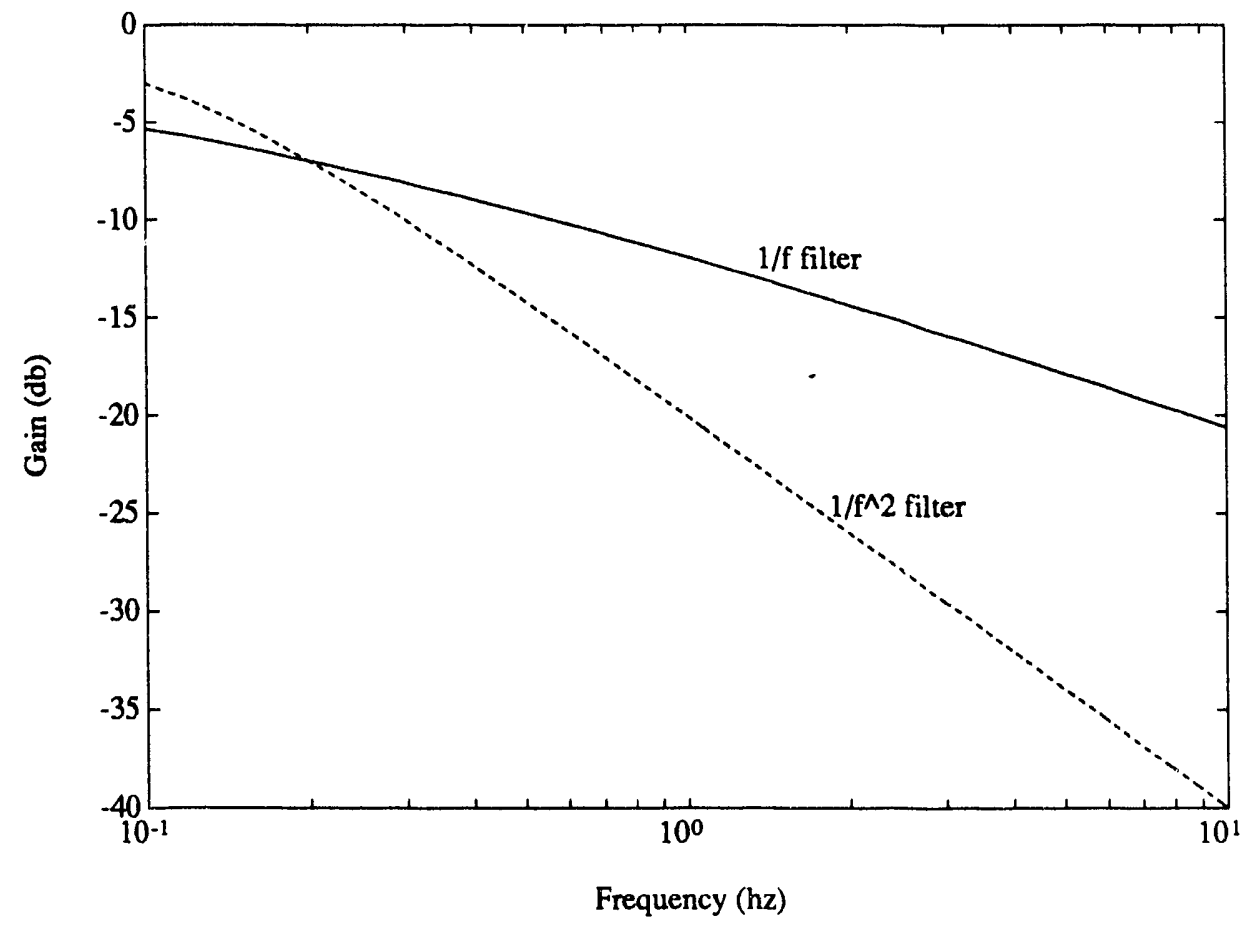

Figure 4.10. Frequency response of $1 / f$ and $1 / f^{2}$ filters. 


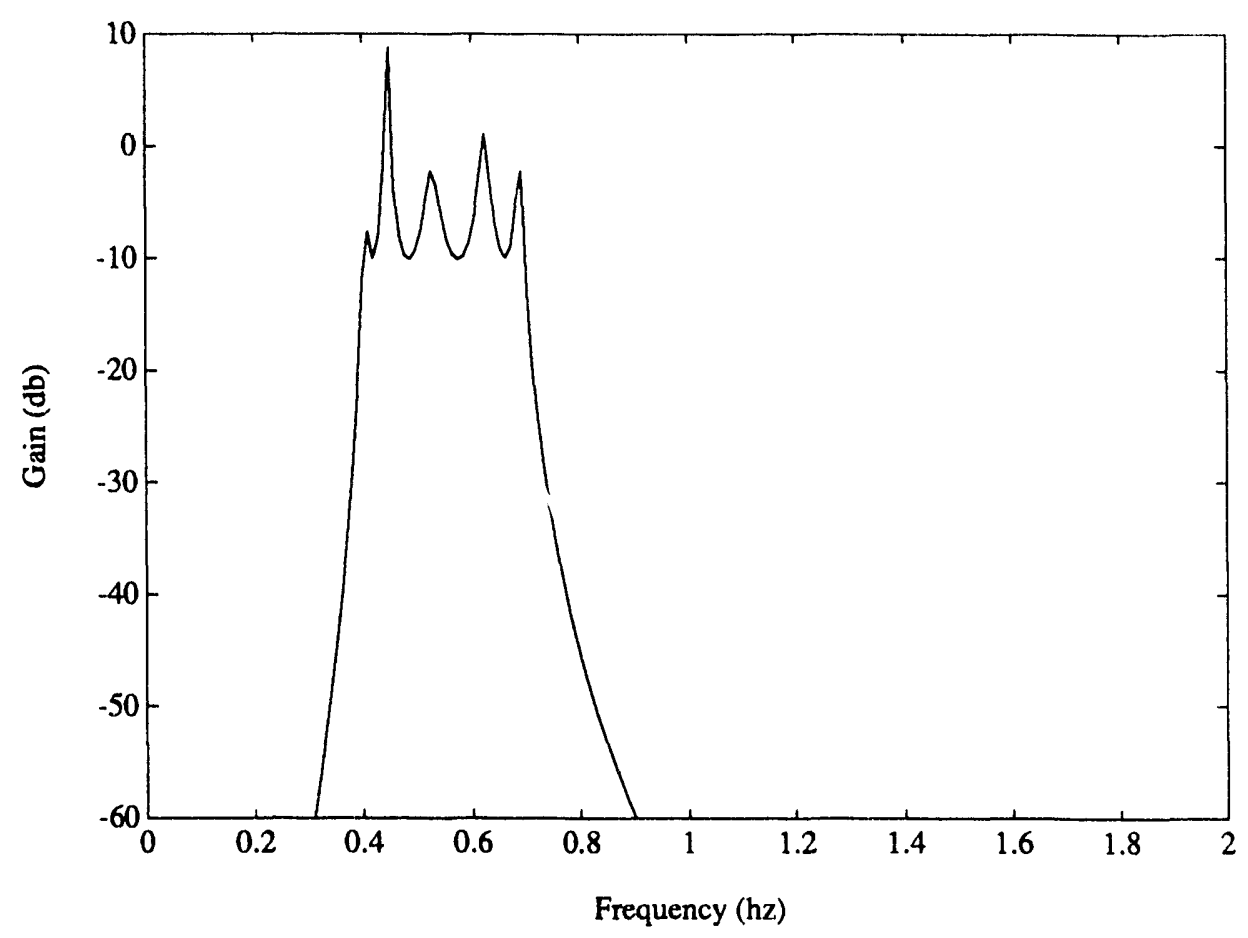

Figure 4.11. Frequency response of coloring filter.

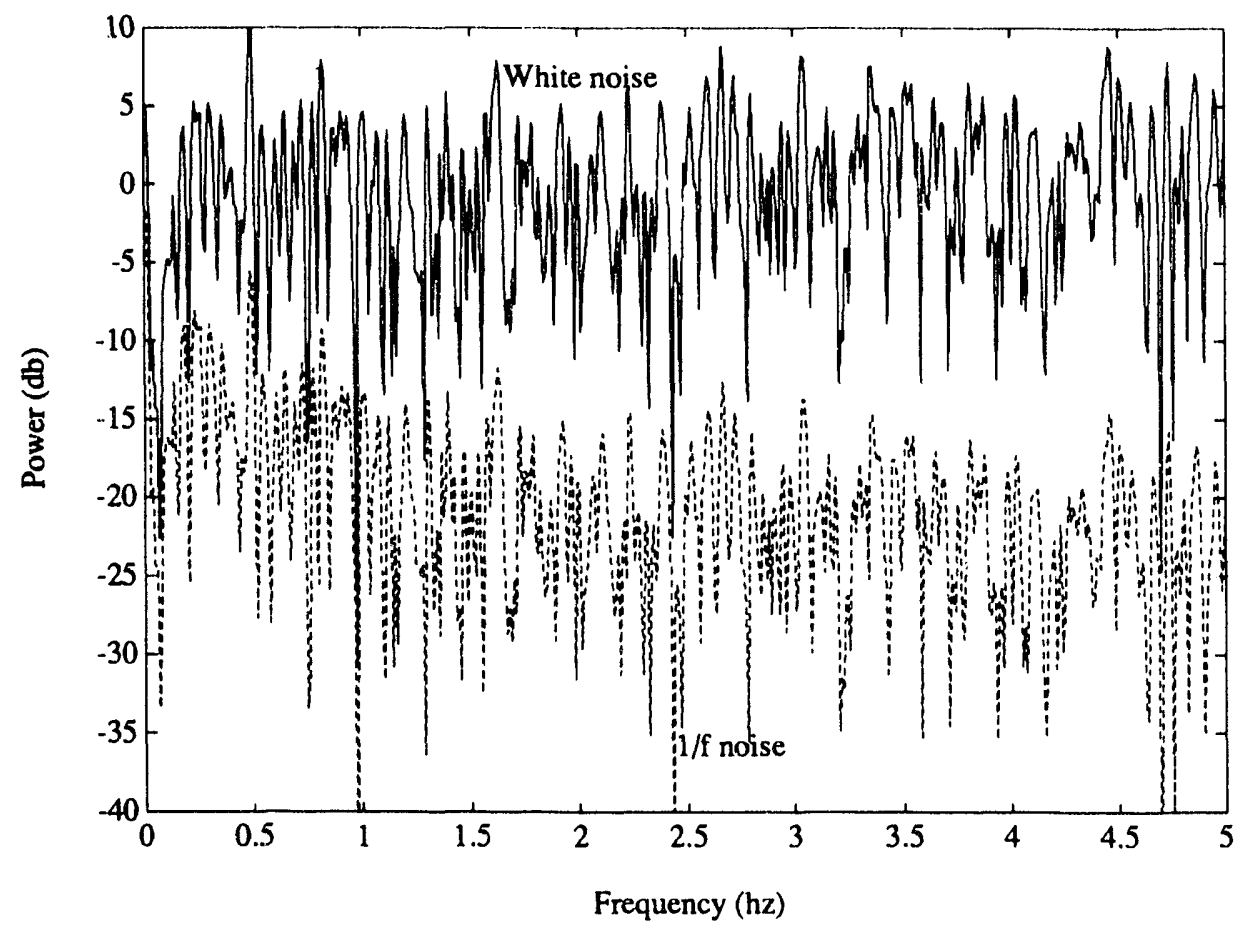

Figure 4.12. Frequency response of white and $1 / f$ noise. 


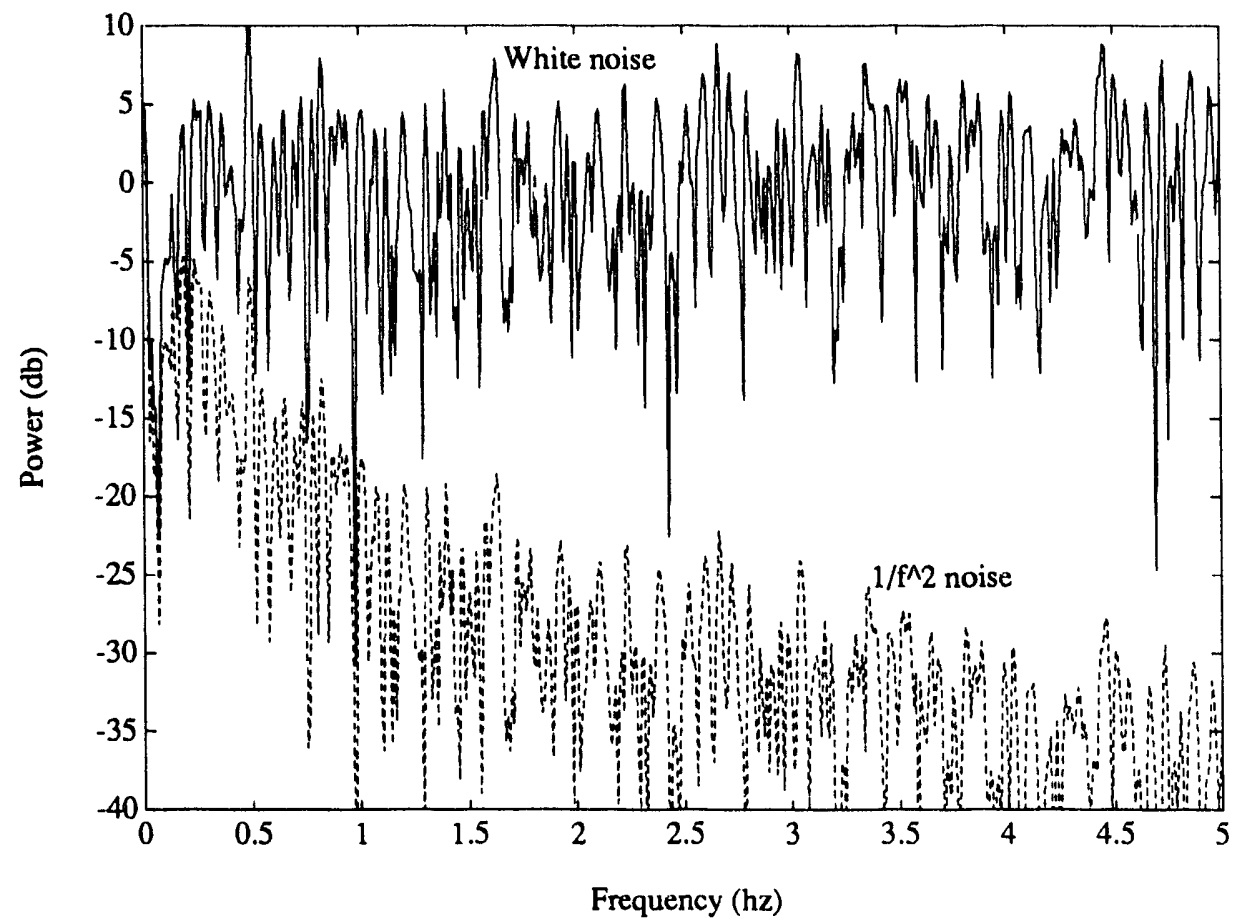

Figure 4.13. Frequency response of white and $1 / f^{2}$ noise.

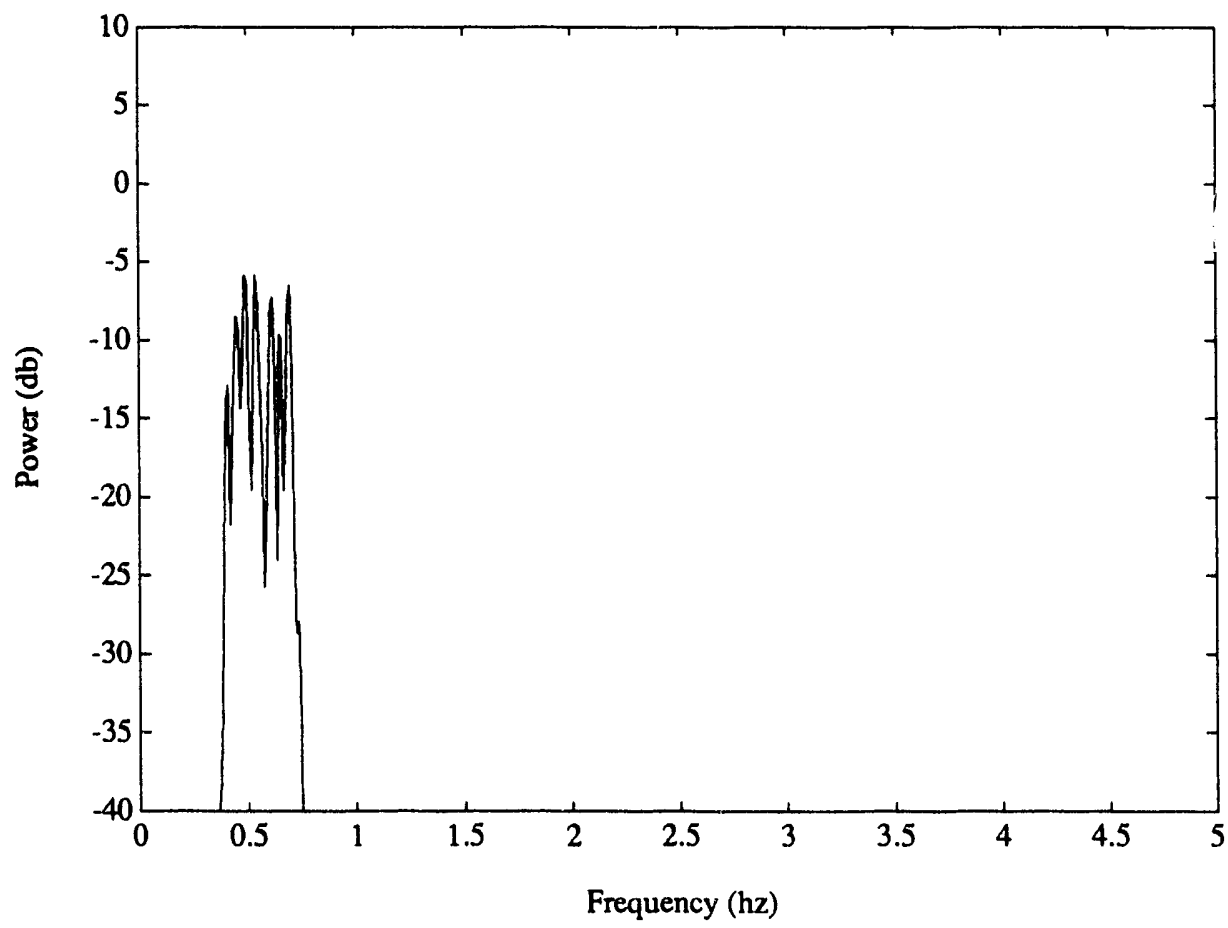

Figure 4.14. Frequency response of colored noise. 


\subsection{Properties of Prony Analysis}

To optimally use an advanced analysis technique such as Prony analysis, one must fully understand its characteristics. These characteristics can then be used to formulate general "rules of thumb" for the technique. In this section, the properties of using Prony analysis to fit linear models to data with known controlled characteristics are discussed.

The properties discussed here were investigated by fitting models to the impulse response of known linear systems with specific characteristics. Special attention is paid to the following effects:

1. alternative solution techniques

2. assumed model order

3. sampling frequency

4. data record length

5. additive noise level and coloration

6. closely-spaced poles

7. closely-spaced pole-zero pairs.

Many tests were conducted using SIGPAKZ and IPRONYID to investigate these properties. Some of the tests are discussed here as examples to demonstrate certain points. The SIGPAKZ input files for the examples are contained in Appendix B.

\subsection{Alternative Solution Techniques}

As discussed in Section 3, PRSPAK offers 288 different solution paths for solving the Prony analysis fitting problem. The options include

1. four methods of formulating the Toeplitz data matrix (termed the LP method)

2. three methods of formulating the LP polynomial (termed the FB method)

3. four choices of LP solution algorithms (termed the LP algorithm)

4. three options for solving the Vandermonde problem.

The only other option available in PRSPAK involves the root finding option. This can be done using either double or quad precision arithmetic. For all the results presented here, the quad-precision routine is used. Other tests not discussed in this report show, that in general, the double-precision routine performs nearly as accurately except for very high-order models (i.e., when $n_{L P}$ is large). It is recommended that one use the quad routine if the respective platform allows; if not, use the double. It seems that in many cases, the double-precision root finder gives waming errors if the polynomial is of 
the order of 50 or more, while the quad typically does not give warnings until order 120 .

\subsubsection{The Linear Prediction Method}

The possible linear prediction methods are correlation, pre-window, covariance, and post-window. Consider a signal $y$ sampled to be $y_{k}, k=0,1, \ldots, N-1$. With the correlation, pre-windowed, and postwindowed options, assumptions must be made concerning the value of $y_{k}$ for $k<0$ and/or $k>N-1$. In PRSPAK the assumption is that $y_{k}=0$ in this range. When analyzing the impulse response of a linear system, the $y_{k}=0$ assumption is the incorrect one because such a system cannot have distinct discontinuities. Therefore, one should expect the covariance method to give more accurate fits than the others for system impulse responses.

Several tests were conducted using the four different LP methods under various system and noise conditions. With all the tests, even the noise-free ones, the covariance fits were considerably better.

\section{Example 5.1}

Consider the test system 1. Fits are conducted on the first 15 seconds of the noise-free impulse response of the system. Table 5.1 compares the pole fits for the different LP methods. Note that the covariance method identifies the parameters much more accurately. The postwindow method gives a relatively accurate estimate, likely because the end of the ring-down signal is close to zero because system damping.

Table 5.1. Identified poles for given LP methods, Example 5.1.

\begin{tabular}{||l|l|l|l|l||}
\hline \multicolumn{1}{|c|}{ Actual } & \multicolumn{1}{c|}{ Cov. } & \multicolumn{1}{c|}{ Corr. } & \multicolumn{1}{c|}{ Pre. } & \multicolumn{1}{c|}{ Post. } \\
\hline$-1.6(2 \pi)$ & $-1.6(2 \pi)$ & Not identified & Not identified & $-1.45(2 \pi)$ \\
$-0.50 \pm j 0.35(2 \pi)$ & $-0.50 \pm j 0.35(2 \pi)$ & $-0.24 \pm j 0.39(2 \pi)$ & $-0.23 \pm j 0.39(2 \pi)$ & $-0.51 \pm j 0.34(2 \pi)$ \\
$-0.25 \pm j 0.45(2 \pi)$ & $-0.25 \pm j 0.45(2 \pi)$ & $-0.15 \pm j 0.46(2 \pi)$ & $-0.14 \pm j 0.46(2 \pi)$ & $-0.27 \pm j 0.45(2 \pi)$ \\
$-0.10 \pm j 0.80(2 \pi)$ & $-0.10 \pm j 0.80(2 \pi)$ & $-0.067 \pm j 0.79(2 \pi)$ & $-0.050 \pm j 0.79(2 \pi)$ & $-0.16 \pm j 0.79(2 \pi)$ \\
$-0.05 \pm j 1.00(2 \pi)$ & $-0.05 \pm j 1.00(2 \pi)$ & $-0.078 \pm j 1.00(2 \pi)$ & $-0.029 \pm j 1.00(2 \pi)$ & $-0.13 \pm j 1.00(2 \pi)$ \\
\hline
\end{tabular}

\subsubsection{Linear Prediction Algorithm}

Once the linear prediction method is determined, a linear prediction algorithm is selected. As described in Section 3, there are four linear prediction algorithms: 1) singular-value decomposition (SVD); 2) standard QR; 3) total least-squares (TLS); and 4) fast QR. All the algorithms provide relatively accurate mode estimates in low-level noise cases. With high-level noise, one algorithm might work slightly better than the others, but the "best" algorithm seems to be highly variable, although SVD is consistently one of the better.

The advantage of the fast $\mathrm{QR}$ algorithm is that it is much faster computationally. As described in Demeure and Scharf (1990), the complexity of the standard Cholesky QR factorization method is on the order of $\left(N+n_{L P}\right) n_{L P}$ while the fast $\mathrm{QR}$ is on the order of $N\left(n_{L P}\right)$, where $N$ is the number of data points. If one uses the recommended assumption that $N / 3<n_{L P}<N / 2$, then the fast $\mathrm{QR}$ can represent significant speed-up. Although no specific timing tests were conducted, in most cases tested for this 
report, the fast QR computed LP terms more than twice as fast as the other algorithms.

\subsubsection{Forward/Backward Formulation}

In formulating the linear prediction equations, one can write the auto-regressive equations in either forward or backward time. PRSPAK allows the use of forward, backward, and a combination of both. The roots of the backward AR polynomial are reflected about the unit circle when compared to the roots of the forward AR polynomial.

Several cases were investigated to compare the three possible formulations. In the noiseless case, all three perform equally well. But as noise is added to the data, the forward option results in significantly more accurate pole estimates.

\section{Example 5.2}

Fits are performed to the impulse of test system 1 with added white noise at a variance of $10(\mathrm{SNR}=17 \mathrm{db})$. Table 5.2 compares the identified $0.35 \mathrm{hz}$ modes for the three cases. The other identified modes have similar characteristics. Note that the forward option results in a much more accurate identified pole. The backward option identifies the 0.35 -hz pole in the right half of the s-plane which is a reflection about the unit circle.

Table 5.2. Identified $0.35-\mathrm{hz}$ terms for forward/backward formulations, Example 5.2.

\begin{tabular}{||c|l|l|l|l|}
\hline & \multicolumn{1}{|c|}{ Actual } & \multicolumn{1}{c|}{ Forward } & \multicolumn{1}{c|}{ Backward } & For./Back. \\
\hline \hline \multirow{2}{*}{ Pole } & -0.50 & -0.52 & 0.73 & -0.30 \\
& $\pm j 0.35(2 \pi)$ & $\pm j 0.36(2 \pi)$ & $\pm j 0.36(2 \pi)$ & $\pm j 0.37(2 \pi)$ \\
\hline
\end{tabular}

\subsubsection{Vandermonde-System Solving Algorithms}

Once the mode estimates have been obtained by solving the linear prediction problem, the signal residues must be calculated. As described in Section 3, PRSPAK offers three options in solving the Vandermonde system: 1) standard QR; 2) fast real; and 3) fast complex. Under the conditions studied, all three give the same results. This includes the analysis of both noiseless and noisy data.

\subsection{Effects of Linear Prediction Model Order}

The literature recommends that one assume the model order to be large to allow extra terms for fitting the "noise" effects; doing so frees the other terms to fit actual system modes. Then the user must determine which identified terms represent noise and which represent actual system modes. For example, it is suggested in Kumaresan and Tufts (1982) that the assumed model order be N/3 to N/2 where $N$ is the number of data points. The results obtained with the tests conducted with SIGPAKZ tend to support this recommendation.

In the noise-free case, model order is not very critical. If the model order is assumed too small, Prony analysis tends to produce a model that "averages through" small response details. In this case 
none of the identified terms is accurate. If the model order is greater than or equal to the true system order, an accurate model is identified with the extraneous terms having very small residues.

As noise is added to the system output, model order becomes more critical. Even for very lowlevel noise, a model order equal to the system order results in a poor fit. As the model order is progressively increased, the fit improves. For low-level noise, a model order near N/5 or greater seems to provide good fits (assuming $\mathrm{N} / 5$ is greater than the true model order).

Separating the system model terms from the noise model terms is a difficult problem. No separation algorithm is currently employed in SIGPAKZ. IPRONYID uses a method recently developed at PNL, and mentioned in Section 2. This subject is discussed further in Section 6.

\section{Example 5.3}

In this example, several fits with various values for $n$ are conducted to the impulse response of the 9th-order test system 1 with added white noise at a variance of 1.0 (SNR $=27$ $\mathrm{db}$ ). Each fit includes 150 data points. Figures 5.1 through 5.6 show the time and frequency response of identified models for $n=3,15$, and 30. Each time-domain plot compares the model with the actual system noisy data, and the frequency-response plots show the "clean" response of the actual system. For $n=3$, the $0.35-\mathrm{hz}$ mode is nearly identified. This is somewhat expected as this is the dominant mode. For $n=15$, the identified model is not very accurate because of the noise effects. Not until $n=30$ does the Prony analysis identify an accurate model. With the 30th-orier model, 9 of the identified modes very closely match that of the actual system; these are used as the identified model. As $n$ is increased past 30, the identified model parameters continue to improve in accuracy.

\subsection{Effects of Sampling Frequency}

It is well known that in sampling continuous-time data for analysis, one must sample at least the Nyquist rate. But, one also must not sample at too high a rate as the Z-transform poles converge to 1 on the unit circle. The natural question is, Given these restrictions, what is the best sample rate to use for optimum parameter identification?

Prony analysis is relatively robust to variations in the sample frequency. A general rule for choosing the sample rate is to make the highest frequency pole occur near the imaginary axis in the Zplane. That is, the sample frequency should be double the Nyquist rate. If the sample frequency is on the order of four time greater than this, one is likely to still obtain accurate results. But, if the rate is two orders of magnitude greater than the general rule, errors are likely to occur because of Z-plane poles converging to 1 . 


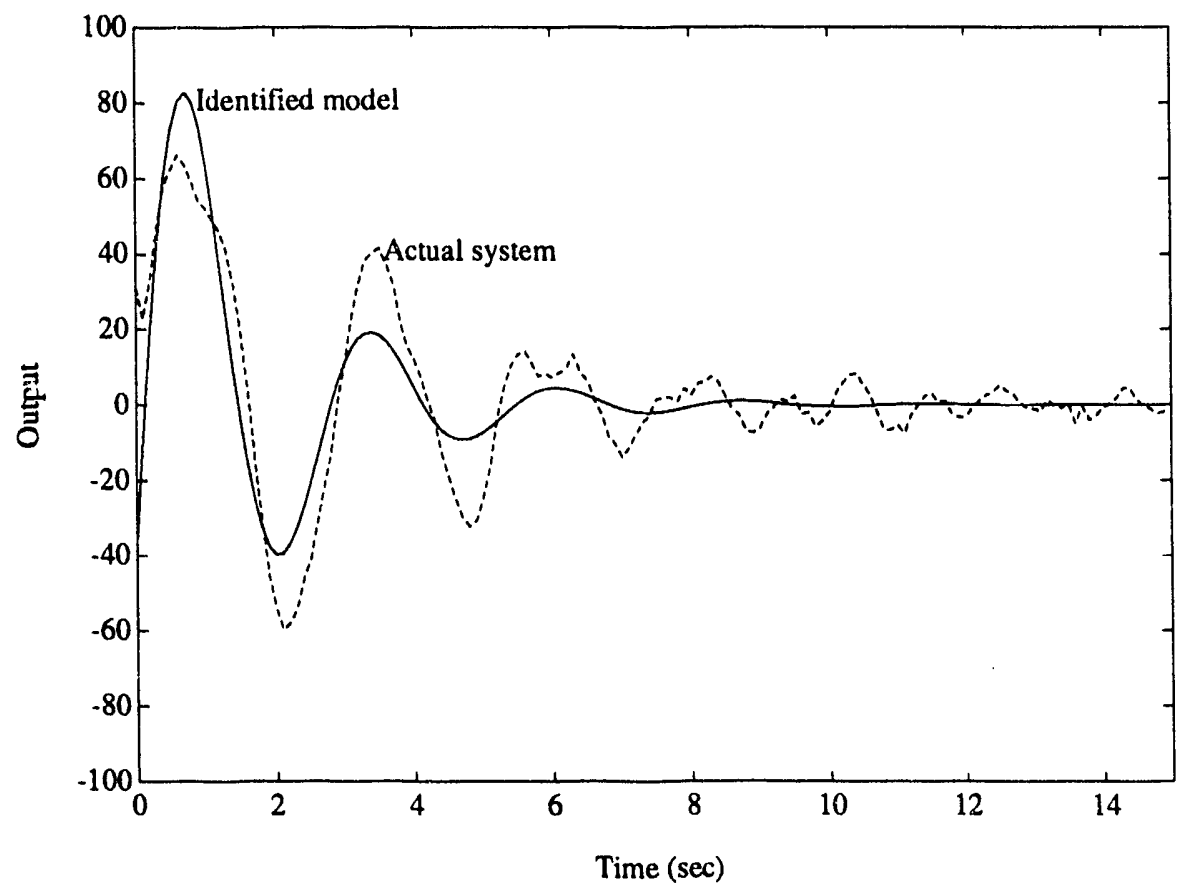

Figure 5.1. Impulse response of 3rd-order fit to 9th-order system with white noise at 27-db SNR, Example 5.3.

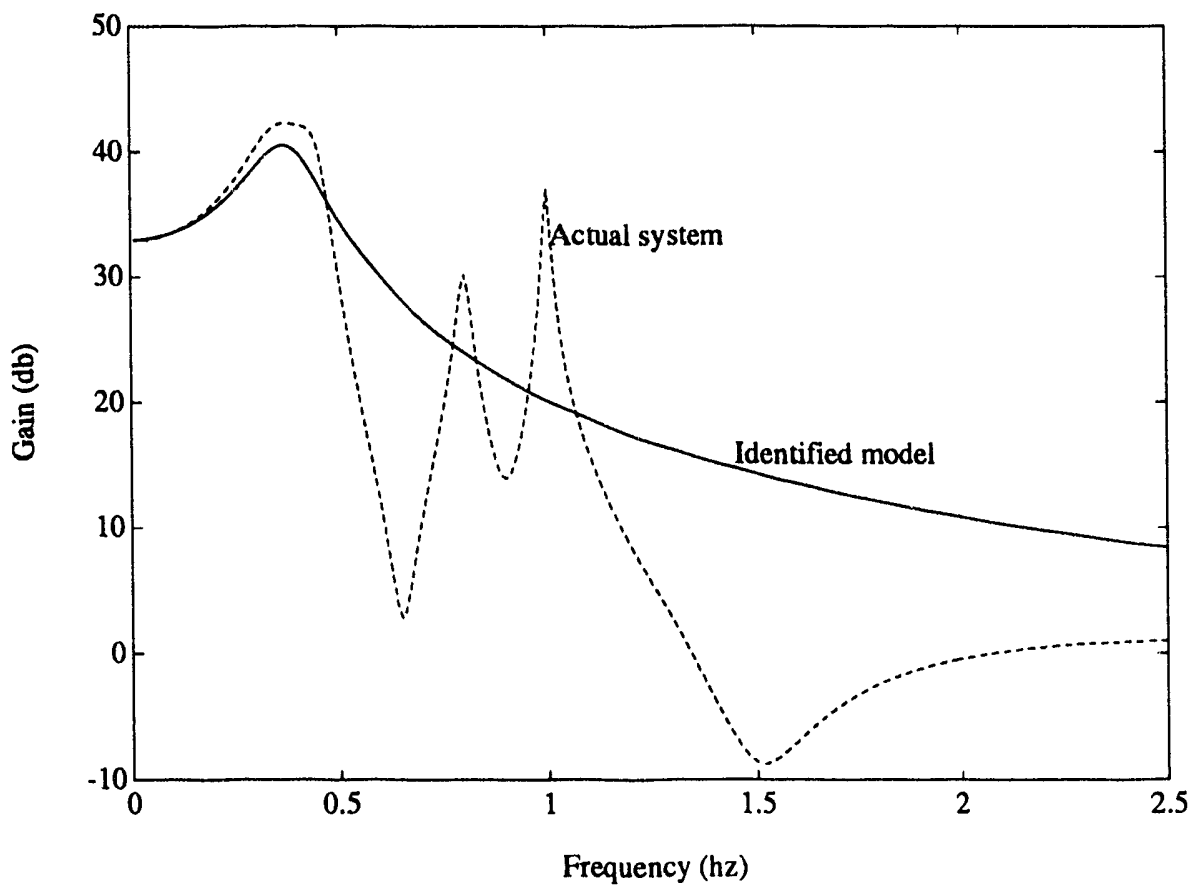

Figure 5.2. Frequency response of 3rd-order fit to 9th-order system with white noise at 27-db SNR, Example 5.3. 


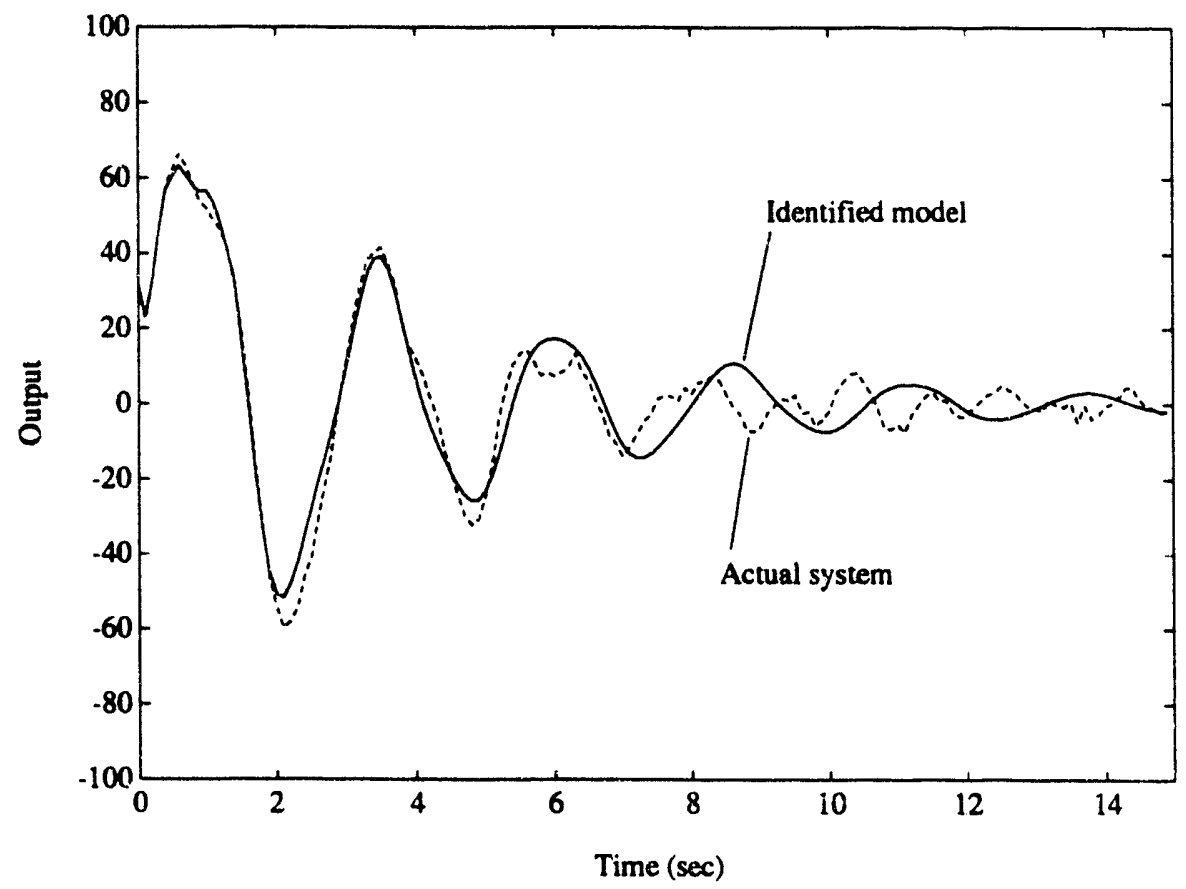

Figure 5.3. Impulse response of 15 th-order fit $1091^{\circ}$-order system with white noise at 27-db SNR, Example 5.3.

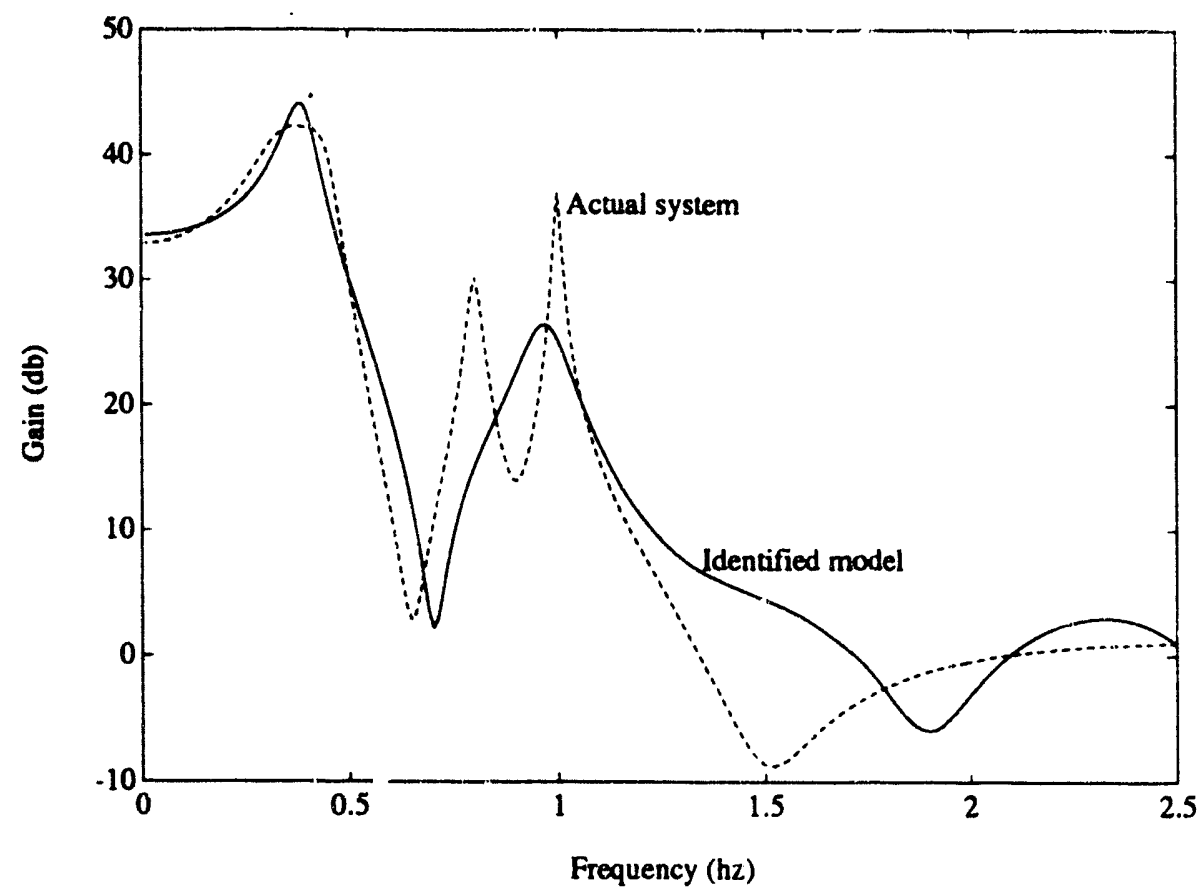

Figure 5.4. Frequency response of 15 th-order fit to 9 th-order system with white noise at 27-db SNR, Example 5.3. 


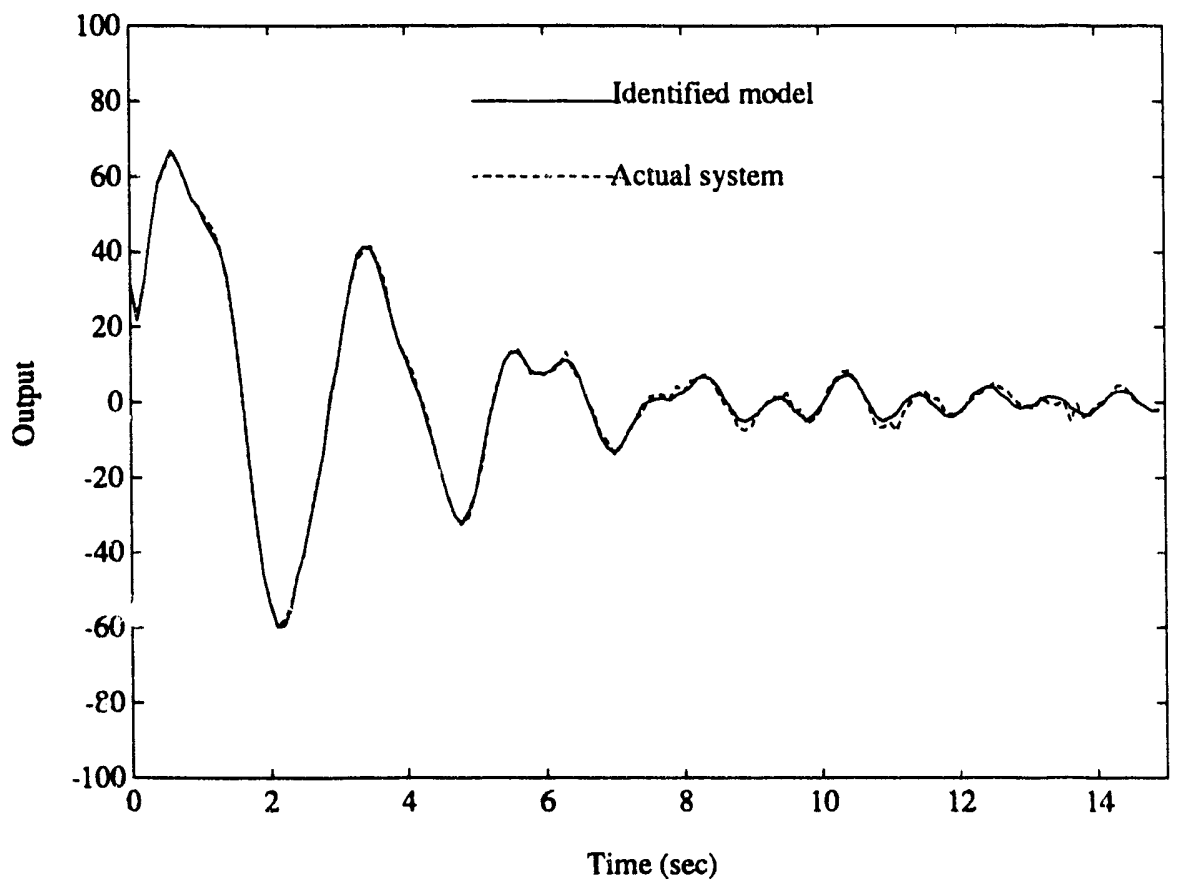

Figure 5.5. Impulse response of 30th-order fit to 9th-order system with white noise at 27-db SNR, Example 5.3.

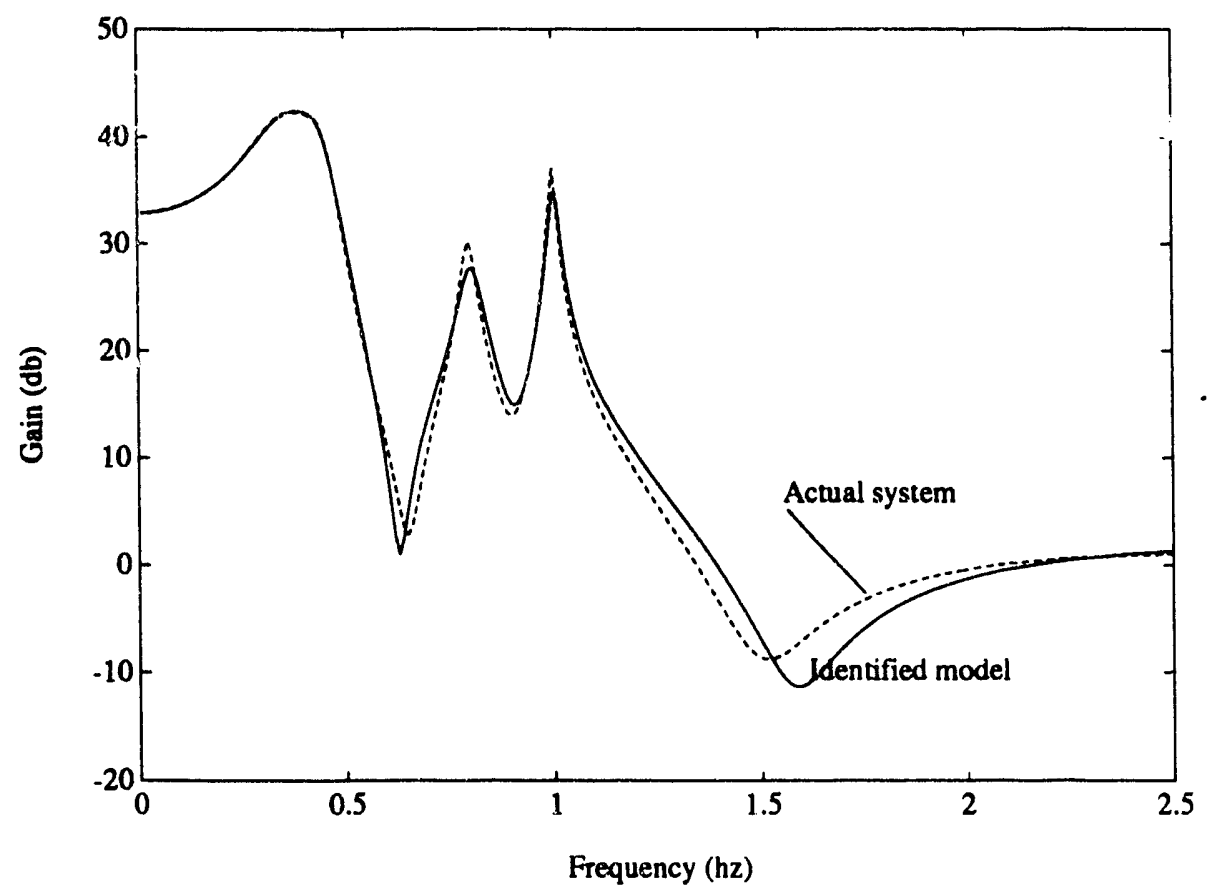

Figure 5.6. Frequency response of 30th-order fit to 9th-order system with white noise at 27-db SNR, Example 5.3. 


\subsection{Effects of Record Length}

Naturally, one should provide as much information as possible to a fitting routine. But, for ringdown signals, at some point the noise will tend to dominate the data. At this point, additional data will likely make the fitting more difficult.

Based on many tests with Prony analysis, it seems that a general rule of thumb is that one should provide enough data to cover at least one full cycle of the low-frequency dominant modes of oscillation. This limit seems to be a corner, a safer rule might be to use two full cycles. Somewhat surprisingly, this rule is fairly independent of the sampling frequency.

\section{Example 5.4}

Fits are performed on varying lengths of the impulse response of test system 2. Figures 5.7 and 5.8 show the impulse and frequency responses of fits performed on 5 seconds of data and 2.5 seconds. The 5-second fit exactly matches the actual system data, but the 2.5 -second fit is very poor.

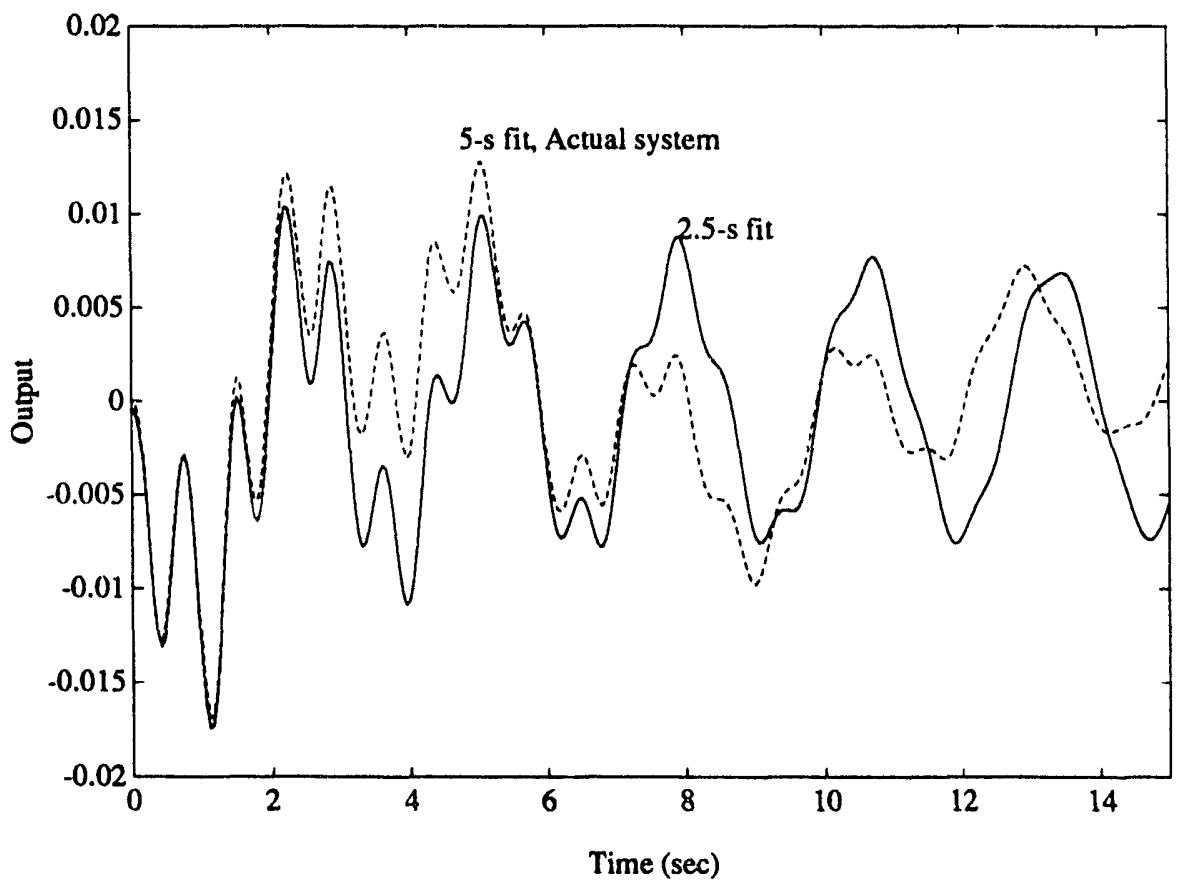

Figure 5.7. Different data record fits impulse response, Example 5.4. 


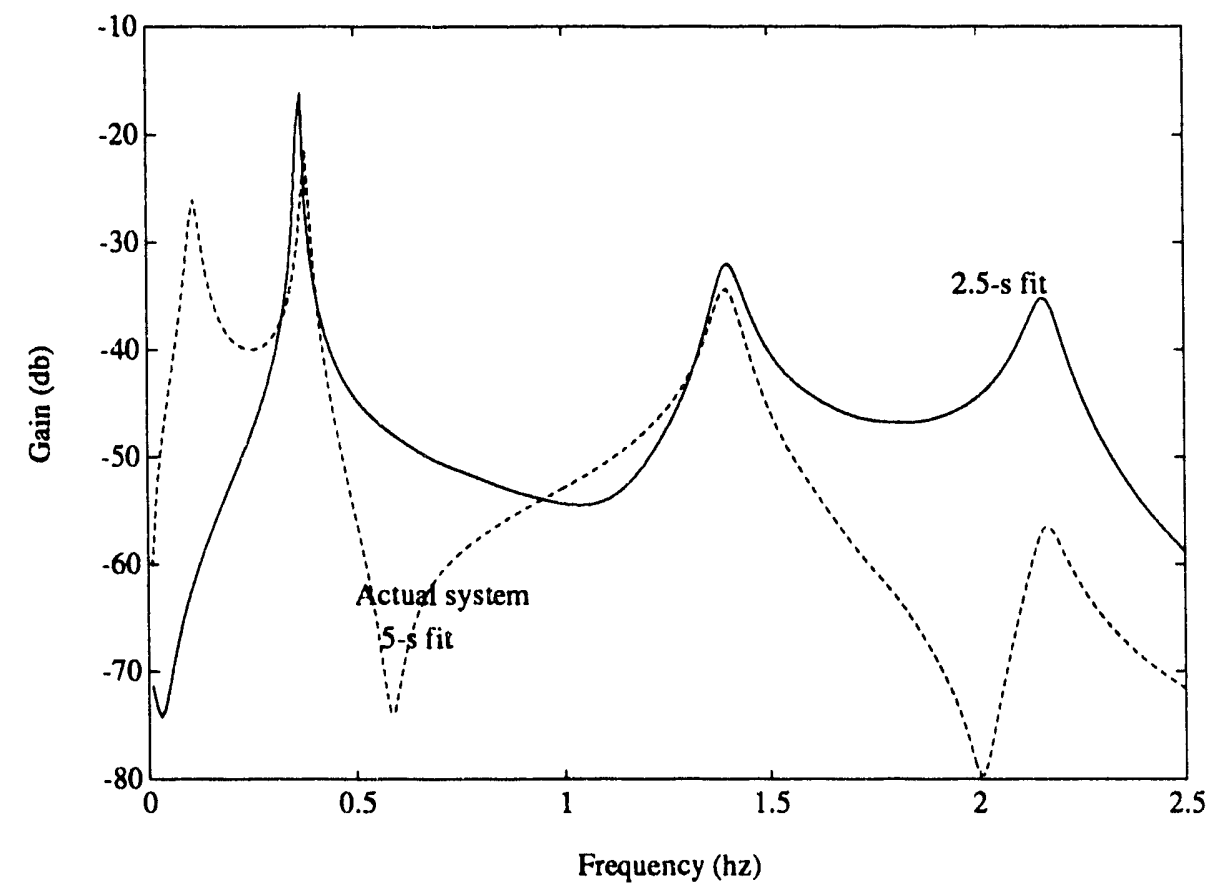

Figure 5.8. Different record length fits frequency response, Example 5.4.

\section{Example 5.5}

Now fits are performed to the noisy (variance $=10$ ) response of test system 1. Figures 5.9 and 5.10 show the model and system responses for 10 -second and 50-second fits. The 10second fit is much more accurate (the identified parameters are also much more accurate). This is because, after 10 seconds, the ringdown is dominated by the noise.

\subsection{Effects of Noise Level and Coloration}

To investigate the effects of additive noise on Prony analysis, the different noise signals described in Section 4 were added to the impulse responses of the test systems. Several noise levels and colorations were tested.

Noise coloration has less effect on the performance of the Prony analysis than the noise level. As expected, poor identification occurs in the bandwidth of the noise. However, in most cases, accurate identification occurs outside the bandwidth of the noise. White and $1 / f$ seem to equally affect all parameters, as these noise types have a wide bandwidth. $1 / f^{2}$ noise tends to cause errors at the lower frequency end, and colored noise causes errors on terms within its bandwidth.

In general, accurate parameter identification is achieved for signal-to-noise ratios of 30-db SNR or more, and in most cases, relatively accurate fits are obtained with a SNR of $20 \mathrm{db}$. Noise levels greater than 10-db SNR cause significant parameter errors. Under high noise conditions, use of a shorter data record can improve the fit (this is demonstrated in Example 5.5). This is because the lower end of the ringdown is dominated by the noise. 


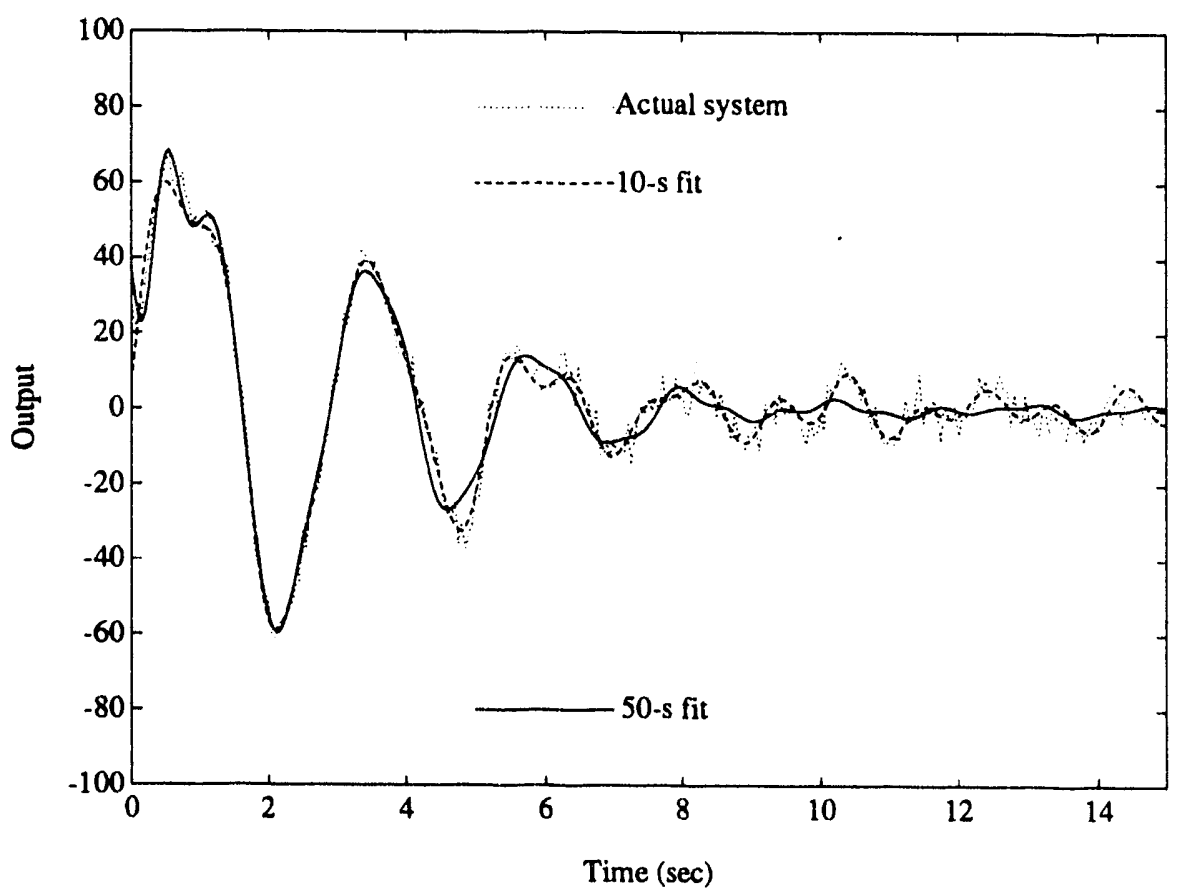

Figure 5.9. Impulse response of different record length fits, Example 5.5.

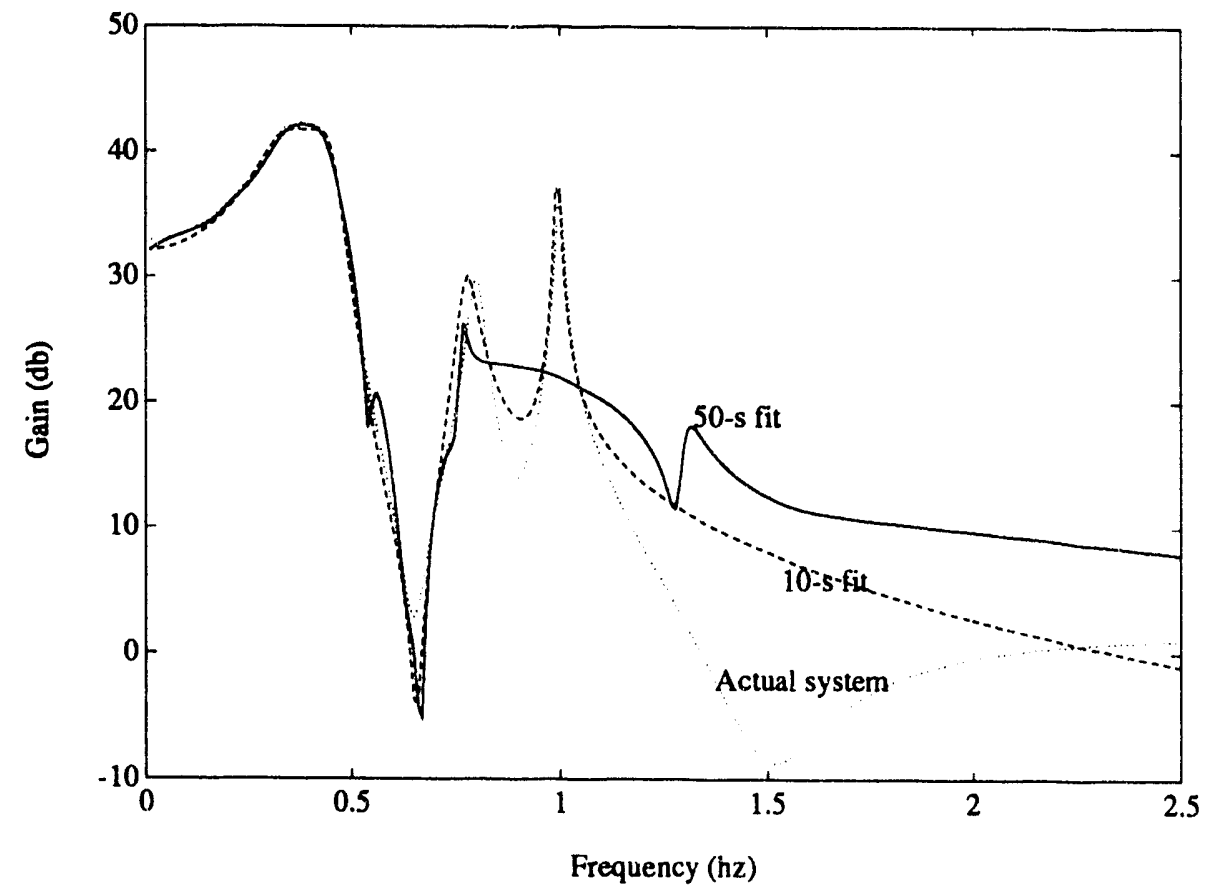

Figure 5.10. Frequency response, different record length fits, Example 5.5. 


\section{Example 5.6}

In this example, progressively higher-level white noise is added to the impulse response of test system 1. Figures 5.11 through 5.13 show the fits for noise levels of variance 0.05 (40-db SNR) and 15 (15-db SNR); 15 seconds of data are used with each fit. The model parameters used to create the plot responses in these figures consist of only the parameters that closely match actual system parameters. The 40-db SNR case results in a very good fit, both graphically and in the parameters. The 15-db SNR fits poorly at the lower frequency gain areas, but appears to fit well at the higher gains (e.g., near $0.35 \mathrm{hz}$ ).

Figures 5.12 and 5.13 suggest that the $15-\mathrm{db}$ SNR fit is accurate near $0.35 \mathrm{hz}$, but the identified parameters are not accurate. The actual system has a pole at $-0.50 \pm j 0.35(2 \pi)$. The Prony analysis identifies two poles in this area; they are at $-41 \pm j 0.33(2 \pi)$ and $-0.28 \pm j$ $0.38(2 \pi)$. Both modes have large residues. This demonstrates that in high noise conditions one can obtain an accurate time and frequency domain fit, but the actual identified parameters may be in error.

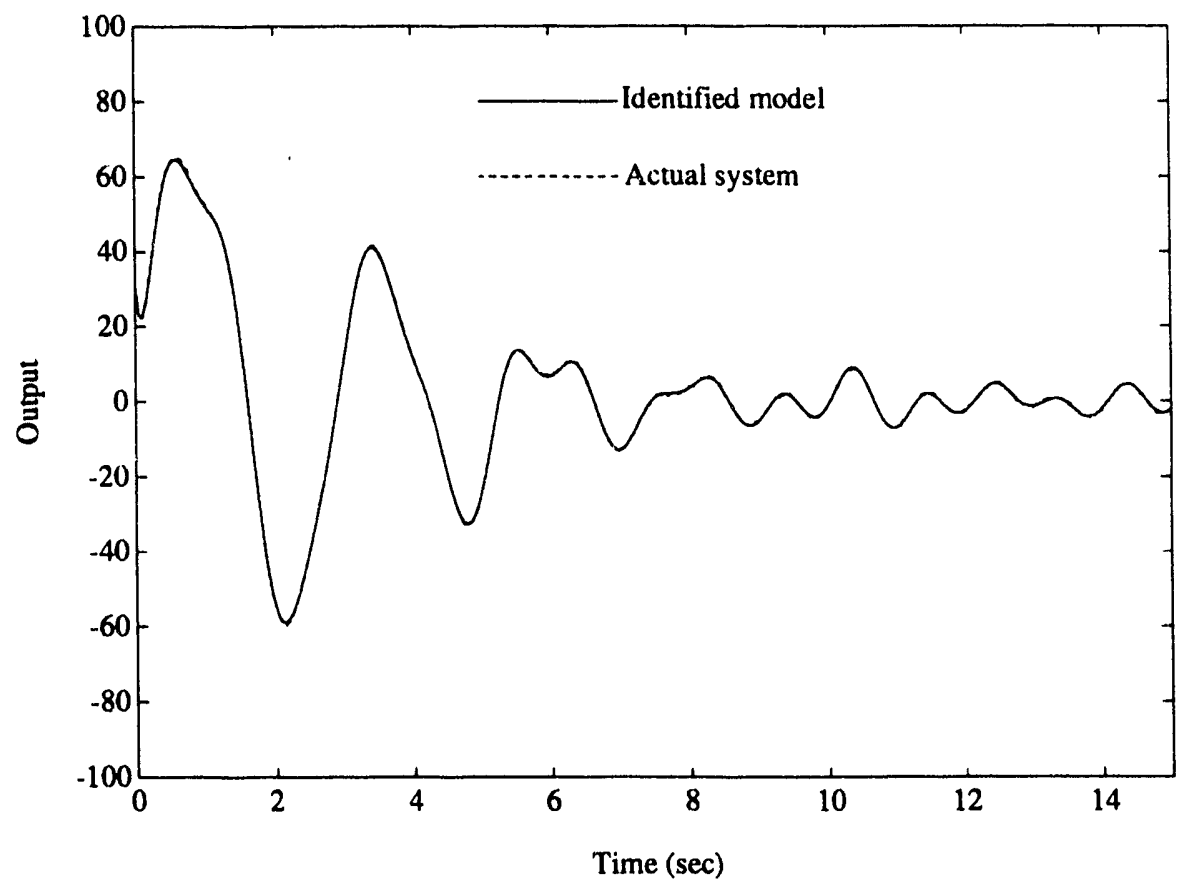

Figure 5.11. Impulse response of fit to system with $40-d b$ SNR, Example 5.6. 


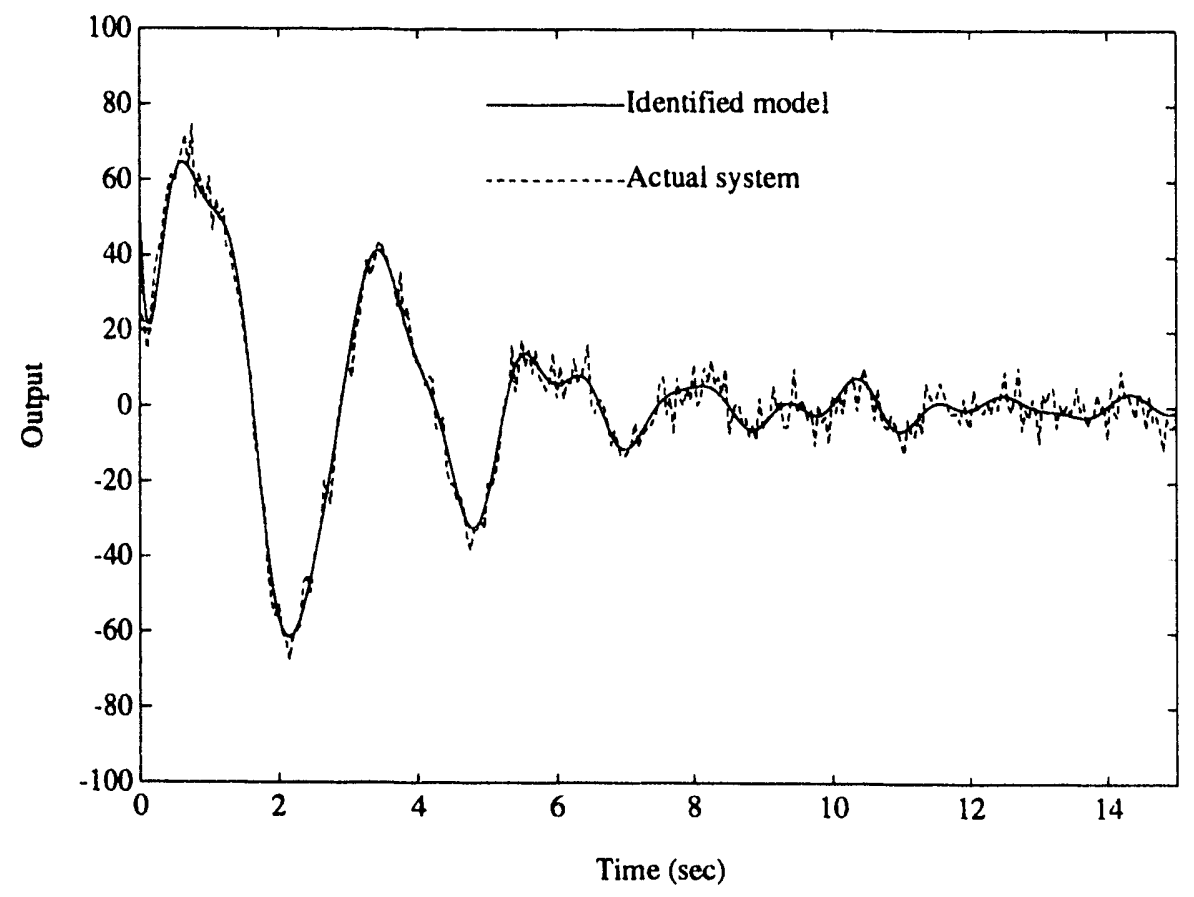

Figure 5.12. Impulse response of fit to system with $15-\mathrm{db}$ SNR, Example 5.6.

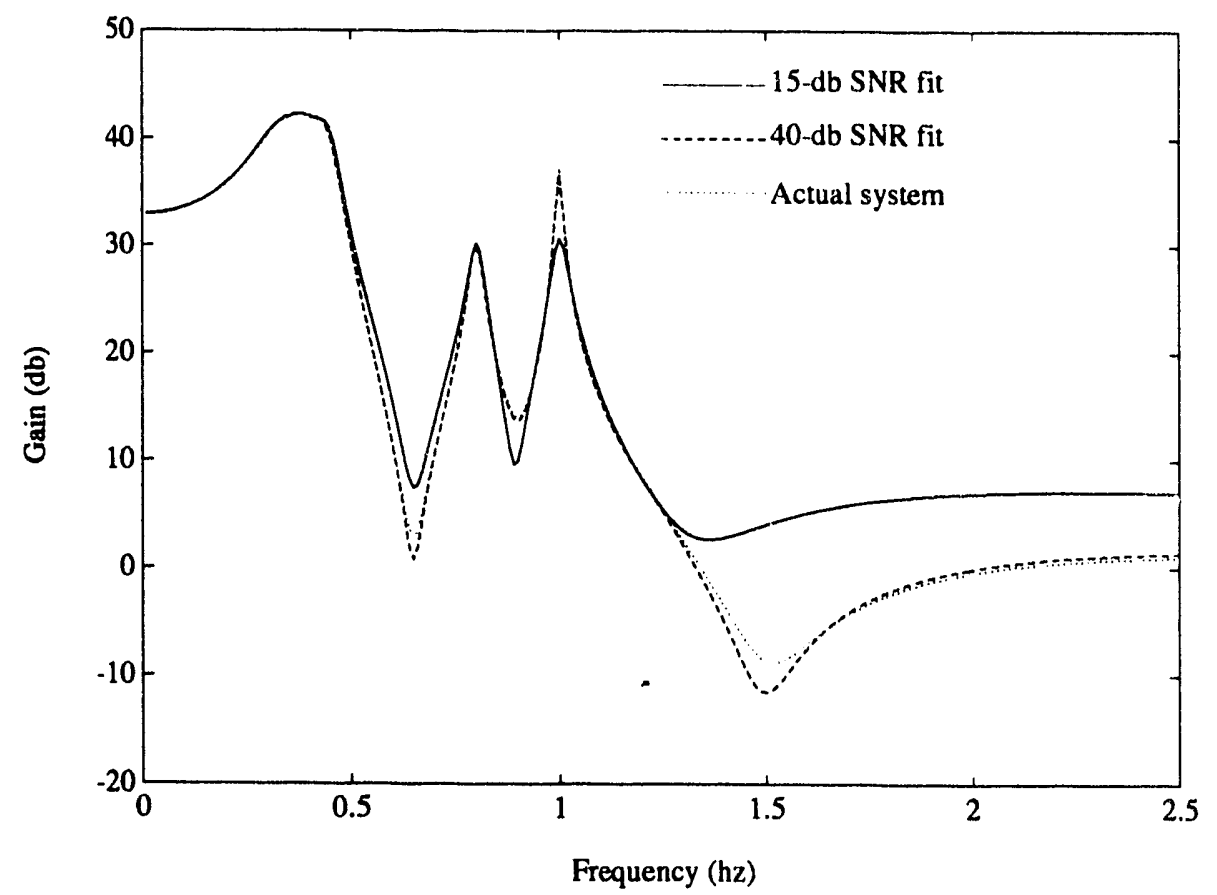

Figure 5.13. Frequency response of different noise level fits, Example 5.6. 


\section{Example 5.7}

In this example, different noise colorations are compared. Four different colorations are added to the impulse response of test system 1 each at a SNR of $20 \mathrm{db}$ (based on a 15 second ringdown). The four colorations are white, $1 / f, 1 / f^{f}$, and sharply colored between $0.4 \mathrm{hz}$ and 0.7 $\mathrm{hz}$ (see Section 4). Table 5.3 shows the identified parameters for each case. Note that the $1 / f^{2}$ noise causes relatively larger errors at the lower frequencies. This is because the energy of such noise is concentrated at the low end. Errors caused by the white and $1 / f$ noise are spread over all the frequencies due to the wide-band effect of the noise. The colored noise caused only slight error for the $0.45-\mathrm{hz}$ mode. In this case, Prony identified other modes in this area to adapt to the colored noise. Parameters outside the bandwidth of the colored noise are accurately identified.

Table 5.3. Identified poles and residues for given colored noise, Example 5.7.

\begin{tabular}{|c|c|c|c|c|}
\hline $\begin{array}{l}\text { Actual } \\
\text { Pole } \\
\text { Residue }\end{array}$ & White & $1 / f$ & $1 / f^{2}$ & Colored \\
\hline $\begin{array}{l}-1.6(2 \pi) \\
44.1\end{array}$ & $\begin{array}{l}-1.57(2 \pi) \\
65.8\end{array}$ & $\begin{array}{l}-2.18(2 \pi) \\
58.0\end{array}$ & Not identified & $\begin{array}{l}-1.60(2 \pi) \\
44.2\end{array}$ \\
\hline $\begin{array}{l}-0.50 \pm j 0.35(2 \pi) \\
57.5 \angle \mp 74.5^{\circ}\end{array}$ & $\begin{array}{l}-0.46 \pm j 0.34(2 \pi) \\
46.6 \angle \mp 80.6^{\circ}\end{array}$ & $\begin{array}{l}-0.51 \pm j 0.36(2 \pi) \\
70.0 \angle \mp 90.8^{\circ}\end{array}$ & $\begin{array}{l}-0.48 \pm j 0.37(2 \pi) \\
62.1 \angle \mp 101.0^{\circ}\end{array}$ & $\begin{array}{l}-0.50 \pm j 0.35(2 \pi) \\
57.5 \angle \mp 73.7^{\circ}\end{array}$ \\
\hline $\begin{array}{l}-0.25 \pm j 0.45(2 \pi) \\
25.1 \angle \pm 139.0^{\circ}\end{array}$ & $\begin{array}{l}-0.23 \pm j 0.45(2 \pi) \\
21.5 \angle \pm 142.0^{\circ}\end{array}$ & $\begin{array}{l}-0.24 \pm j 0.46(2 \pi) \\
20.5 \angle \pm 111.0^{\circ}\end{array}$ & $\begin{array}{l}-0.20 \pm j 0.46(2 \pi) \\
16.8 \angle \pm 117.0^{\circ}\end{array}$ & $\begin{array}{l}-0.29 \pm j 0.44(2 \pi) \\
23.3 \angle \pm 146.0^{\circ}\end{array}$ \\
\hline $\begin{array}{l}-0.10 \pm j 0.80(2 \pi) \\
3.00 \angle \mp 110.0^{\circ}\end{array}$ & $\begin{array}{l}-0.11 \pm j 0.80(2 \pi) \\
3.49 \angle \mp 116.0^{\circ}\end{array}$ & $\begin{array}{l}-0.12 \pm j 0.80(2 \pi) \\
3.68 \angle \mp 117.0^{\circ}\end{array}$ & $\begin{array}{l}-0.12+j 0.80(2 \pi) \\
3.42 \angle \mp 112.0^{\circ}\end{array}$ & $\begin{array}{l}-0.10 \pm j 0.80(2 \pi) \\
3.04 \angle \mp 110.0^{\circ}\end{array}$ \\
\hline $\begin{array}{l}-0.05 \pm j 1.00(2 \pi) \\
3.42 \angle \mp 143.0^{\circ}\end{array}$ & $\begin{array}{l}-0.085 \pm j 1.00(2 \pi) \\
3.78 \angle x 145.0^{\circ}\end{array}$ & $\begin{array}{l}-0.078 \pm j 1.00(2 \pi) \\
3.53 \angle \mp 146.0^{\circ}\end{array}$ & $\begin{array}{l}-0.05+j 1.00(2 \pi) \\
3.39 \angle \mp 143.0^{\circ}\end{array}$ & $\begin{array}{l}-0.05 \pm j 1.00(2 \pi) \\
3.43 \angle \mp 143.0^{\circ}\end{array}$ \\
\hline
\end{tabular}

\subsection{Effects of Closely-Spaced Poles}

The standard assumed model for Prony analysis does not allow repeated poles. Given this, a natural question is, What is the effect of nearly repeated poles on the fitting algorithms? To investigate these effects, poles were incrementally moved closer together. Two cases were investigated. With one case, the poles were moved closer with equal imaginary terms. In the other, case they were moved closer with equal real terms.

In a linear system as the poles converge, the residues have large, nearly-equal magnitudes but opposite phase. Consider a strictly proper system written in the Laplace domain as

$$
G(s)=\sum_{i=1}^{n} \frac{R_{i}}{s-\lambda_{i}}=\frac{b_{0}\left(s-z_{1}\right)\left(s-z_{2}\right) \ldots\left(s-z_{m}\right)}{\left(s-\lambda_{1}\right)\left(s-\lambda_{2}\right) \ldots\left(s-\lambda_{n}\right)}
$$


where $m<n$, and

$$
\lambda_{2}=\lambda_{1}+\delta
$$

Using partial traction expansion, solve for $R_{l}$ and $R_{2}$ :

$$
\begin{aligned}
& R_{1}=\frac{1}{\delta} \frac{\prod_{i=1}^{m}\left(\lambda_{1}-z_{i}\right)}{\prod_{i=3}^{n}\left(\lambda_{1}-\lambda_{i}\right)} \\
& R_{2}=-\frac{1}{\delta} \frac{\prod_{i=1}^{m}\left(\lambda_{2}-z_{i}\right)}{\prod_{i=3}^{n}\left(\lambda_{2}-\lambda_{i}\right)}
\end{aligned}
$$

Poles $\lambda_{1}$ and $\lambda_{2}$ are moved close together by making $\delta$ small. From (5.3) and (5.4), we see that $R_{l}$ and $R_{2}$ become large in magnitude and opposite in phase. These effects make closely-spaced pole identification relatively difficult.

As one might expect, closely-spaced poles can cause significant problems with Prony analysis fitting. In noise-free cases, Prony analysis performs very well. The closely-spaced terms are identified with less than $1.0 \%$ errors. But, as noise is added to the impulse response, the results quickly change. Even for low-level noise (e.g., 30-db SNR), the errors on the closely-spaced terms significantly increase. The errors are especially large for the identified residues. These results correspond with the sensitivity results presented in McWhorter and Scharf (1991). In many cases, the errors are largest when the poles have similar frequencies but different damping. This is likely because such a configuration can often nearly be represented as a single mode with a compromised damping.

\section{Example 5.8}

Consider the system in Equation (5.5), which is a variation of test system 1.

$$
\left.\begin{array}{rl}
G(s)= & \left(\begin{array}{l}
\left.\frac{s^{2}+0.2 s+0.1^{2}+(3 \pi)^{2}}{s^{2}+0.1 s+0.05^{2}+(2 \pi)^{2}}\right) \\
\frac{s^{2}+0.3 s+0.15^{2}+(1.3 \pi)^{2}}{s^{2}+0.5 s+0.25^{2}+(0.9 \pi)^{2}}
\end{array}\right)\left(\frac{9.6 \pi}{s+3.2 \pi}\right) \\
\frac{s^{2}+1.6 s+0.8^{2}+(1.1 \pi)^{2}}{s^{2}+2(0.5-p 0.25) s+(0.5-p 0.25)^{2}+(0.9 \pi)^{2}}
\end{array}\right)
$$


As $p$ is varied from 0 to 1 , the poles at $0.45 \mathrm{hz}$ converge. Figures 5.14 and 5.15 show the impulse and frequency response of Equation (5.5) for $p=0,0.75$, and 1.0. White noise at a variance of 0.005 ( $30 \mathrm{db} \mathrm{SNR}$ ) is added to the impulse of (5.5) for various $p$ 's, and Prony analysis fits are conducted. Tables 5.4 and 5.5 show the identified parameters for $p=0,0.75$, and 0.9 ; the closely spaced terms are in bold.

The errors on the identified poles are largest for those that are closely spaced; but, errors are less than $20 \%$ in most cases. Note that the damping on one of the closely-spaced poles is too small, while the damping on the other is too large. The residue errors for the closely-spaced poles are very large, especially as $p$ increases. For $p=0.9$, the identified residues are approximately one-sixth of the actual residues. From the large residue errors, one might expect the time and frequency domain fits to be poor. This is not the case. Figures 5.16 and 5.17 compare the response of the identified model with the actual system for $p=0.9$. The actual time domain data includes the added noise, while the actual frequency response data does not include the noise. The time-domain fit is very close. The frequency-domain fit is very close at the response peaks. In the frequency range of the closely-spaced poles, the identified model's frequency response very closely matches the actual system response.

Table 5.4. Identified poles, Example 5.8.

\begin{tabular}{|c|c|c|c|c|c|}
\hline $\begin{array}{l}\text { Actual } \\
p=0.0\end{array}$ & $\begin{array}{c}\text { Identified } \\
\mathrm{p}=0.0\end{array}$ & $\begin{array}{c}\text { Actual } \\
p=0.75\end{array}$ & $\begin{array}{l}\text { Identified } \\
p=0.75\end{array}$ & $\begin{array}{l}\text { Actual } \\
p=0.9\end{array}$ & $\begin{array}{c}\text { Identified } \\
\mathrm{p}=0.9\end{array}$ \\
\hline$-1.6(2 \pi)$ & $-2.280(2 \pi)$ & $-1.6(2 \pi)$ & $-2.09(2 \pi)$ & $-1.6(2 \pi)$ & $-2.06(2 \pi)$ \\
\hline $\begin{array}{l}-0.25 \\
+j 0.45(2 \pi) \\
\end{array}$ & $\begin{array}{l}-0.242 \\
\pm j 0.445(2 \pi) \\
\end{array}$ & $\begin{array}{l}-0.25 \\
+j 0.45(2 \pi) \\
\end{array}$ & $\begin{array}{l}-0.220 \\
+j 0.444(2 \pi) \\
\end{array}$ & $\begin{array}{l}-0.25 \\
\pm j 0.45(2 \pi) \\
\end{array}$ & $\begin{array}{l}-0.209 \\
+j 0.443(2 \pi) \\
\end{array}$ \\
\hline $\begin{array}{l}-0.5 \\
+j 0.45(2 \pi) \\
\end{array}$ & $\begin{array}{l}-0.566 \\
\pm j 0.464(2 \pi) \\
\end{array}$ & $\begin{array}{l}-0.3125 \\
+j 0.45(2 \pi) \\
\end{array}$ & $\begin{array}{l}-0.364 \\
+j 0.461(2 \pi) \\
\end{array}$ & $\begin{array}{l}-0.275 \\
+j 0.45(2 \pi) \\
\end{array}$ & $\begin{array}{l}-0.333 \\
\pm j 0.460(2 \pi) \\
\end{array}$ \\
\hline $\begin{array}{l}-0.10 \\
+0.80(2 \pi) \\
\end{array}$ & $\begin{array}{l}-0.105 \\
\pm 0.798(2 \pi) \\
\end{array}$ & $\begin{array}{l}-0.10 \\
+j 0.80(2 \pi) \\
\end{array}$ & $\begin{array}{l}-0.105 \\
+j 0.798(2 \pi) \\
\end{array}$ & $\begin{array}{l}-0.10 \\
\pm 0.80(2 \pi) \\
\end{array}$ & $\begin{array}{l}-0.105 \\
\pm j 0.798(2 \pi)\end{array}$ \\
\hline $\begin{array}{l}-0.05 \\
+j 1.00(2 \pi)\end{array}$ & $\begin{array}{l}-0.0521 \\
\pm j 1.00(2 \pi)\end{array}$ & $\begin{array}{l}-0.05 \\
\pm j 1.00(2 \pi)\end{array}$ & $\begin{array}{l}-0.0527 \\
\pm j 1.00(2 \pi)\end{array}$ & $\begin{array}{l}-0.05 \\
\pm j 1.00(2 \pi)\end{array}$ & $\begin{array}{l}-0.0528 \\
\pm j 1.00(2 \pi)\end{array}$ \\
\hline
\end{tabular}

\subsection{Effects of Closely-Spaced Pole-Zero Pairs}

This effect was tested by incrementally moving a pole-zero pair together. As with closely-spaced poles, the terms were converged in two cases: equal real terms and equal imaginary terms. In the noise-free case, Prony analysis performs very well. But as low-level noise is added, the results change significantly. Much more accurate identification is obtained in the case where the closely-spaced polezeros have equal real terms and different imaginary terms. With the other case, the zero appears to provide "false damping" to the close pole. That is, a pole is identified with increased damping to compensate for the zero with the same frequency. 
Table 5.5. Identified residues, Example 5.8.

\begin{tabular}{|c|c|c|c|c|c|}
\hline $\begin{array}{l}\text { Actual } \\
p=0.0\end{array}$ & $\begin{array}{c}\text { Identified } \\
\mathrm{p}=0.0\end{array}$ & $\begin{array}{c}\text { Actual } \\
\mathrm{p}=0.75\end{array}$ & $\begin{array}{l}\text { Identified } \\
\mathrm{p}=0.75\end{array}$ & $\begin{array}{l}\text { Actual } \\
p=0.9\end{array}$ & $\begin{array}{c}\text { Identified } \\
\mathrm{p}=0.9\end{array}$ \\
\hline 4.27 & 4.43 & 4.12 & 4.04 & 4.09 & 3.98 \\
\hline $\begin{array}{l}6.04 \\
\angle \mp 152^{\circ}\end{array}$ & $\begin{array}{l}5.29 \\
\angle \mp 135^{\circ}\end{array}$ & $\begin{array}{l}24.2 \\
\angle \mp 154^{\circ}\end{array}$ & $\begin{array}{l}9.81 \\
\angle \mp 118^{\circ}\end{array}$ & $\begin{array}{l}60.4 \\
\angle \mp 154^{\circ}\end{array}$ & $\begin{array}{l}10.9 \\
\angle \mp 114^{\circ}\end{array}$ \\
\hline $\begin{array}{l}5.18 \\
\angle \pm 2.84^{\circ}\end{array}$ & $\begin{array}{l}3.84 \\
\angle \pm 16.7^{\circ} \\
\end{array}$ & $\begin{array}{l}23.1 \\
\angle \pm 20.2^{\circ} \\
\end{array}$ & $\begin{array}{l}7.6 \\
\angle \pm 52.6^{\circ} \\
\end{array}$ & $\begin{array}{l}59.3 \\
\angle \pm 23.3^{\circ} \\
\end{array}$ & $\begin{array}{l}8.60 \\
\angle \pm 58.7^{\circ} \\
\end{array}$ \\
\hline $\begin{array}{l}0.425 \\
\angle \mp 92.8^{\circ}\end{array}$ & $\begin{array}{l}0.42 \\
6 \angle \mp 89.8^{\circ}\end{array}$ & $\begin{array}{l}0.43 \\
0 \angle \mp 99.0^{\circ}\end{array}$ & $\begin{array}{l}0.432 \\
\angle \mp 96.3^{\circ}\end{array}$ & $\begin{array}{l}0.431 \\
\angle \mp 100^{\circ}\end{array}$ & $\begin{array}{l}0.433 \\
\angle \mp 97.6^{\circ}\end{array}$ \\
\hline $\begin{array}{l}0.455 \\
\angle \mp 170^{\circ}\end{array}$ & $\begin{array}{l}0.461 \\
\angle \pm 171^{\circ}\end{array}$ & $\begin{array}{l}0.458 \\
\angle \mp 175^{\circ}\end{array}$ & $\begin{array}{l}0.466 \\
\angle \mp 176^{\circ}\end{array}$ & $\begin{array}{l}0.458 \\
\angle \mp 175^{\circ}\end{array}$ & $\begin{array}{l}0.467 \\
\angle \mp 177^{\circ}\end{array}$ \\
\hline
\end{tabular}

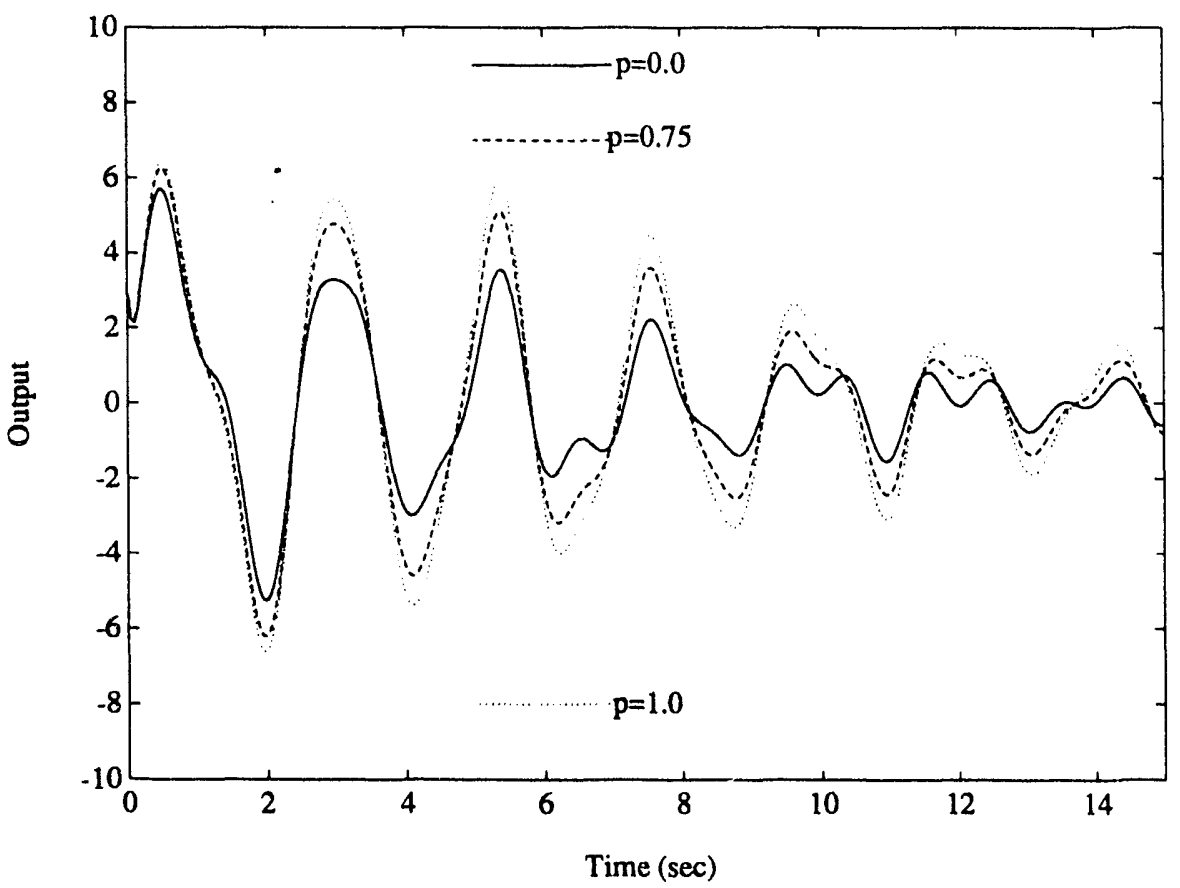

Figure 5.14. Impulse response of system (5.5), Example 5.8 


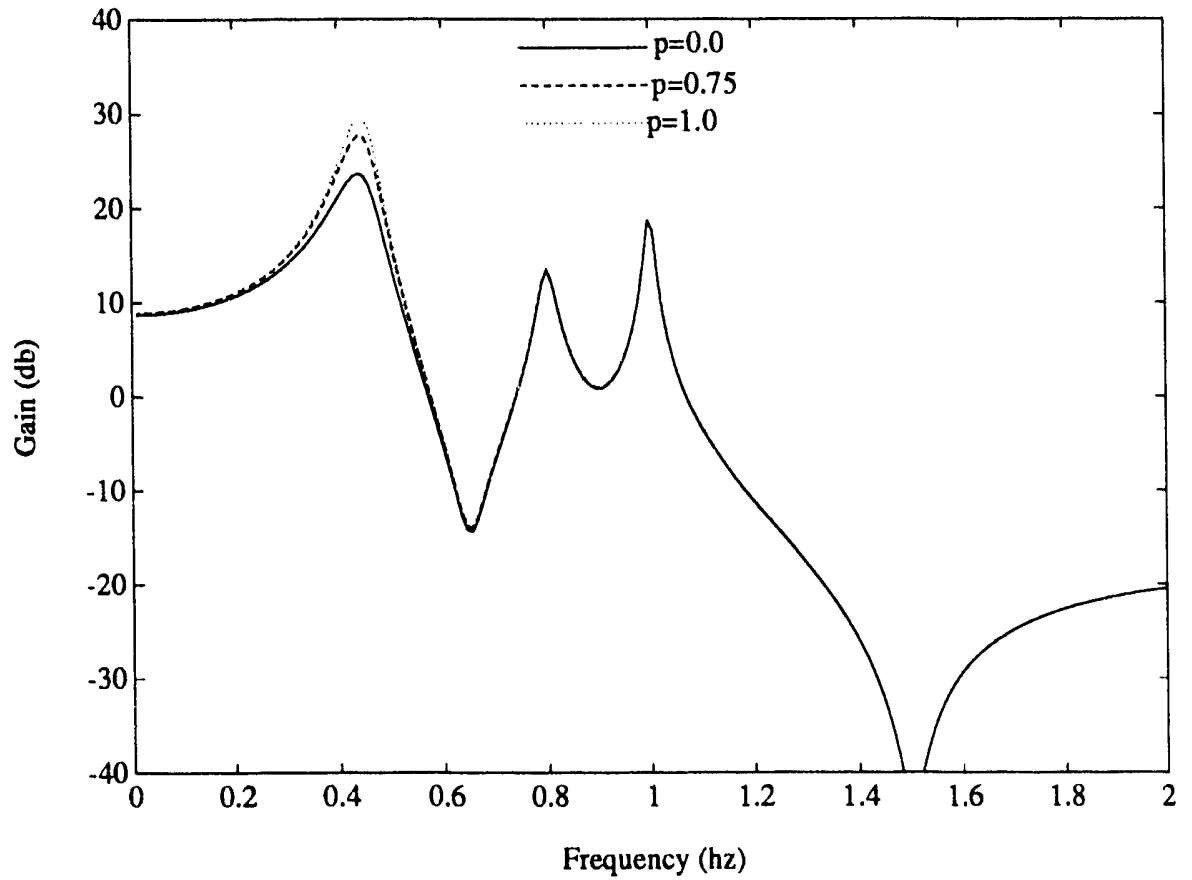

Figure 5.15. Frequency response of system (5.5), Example 5.8.

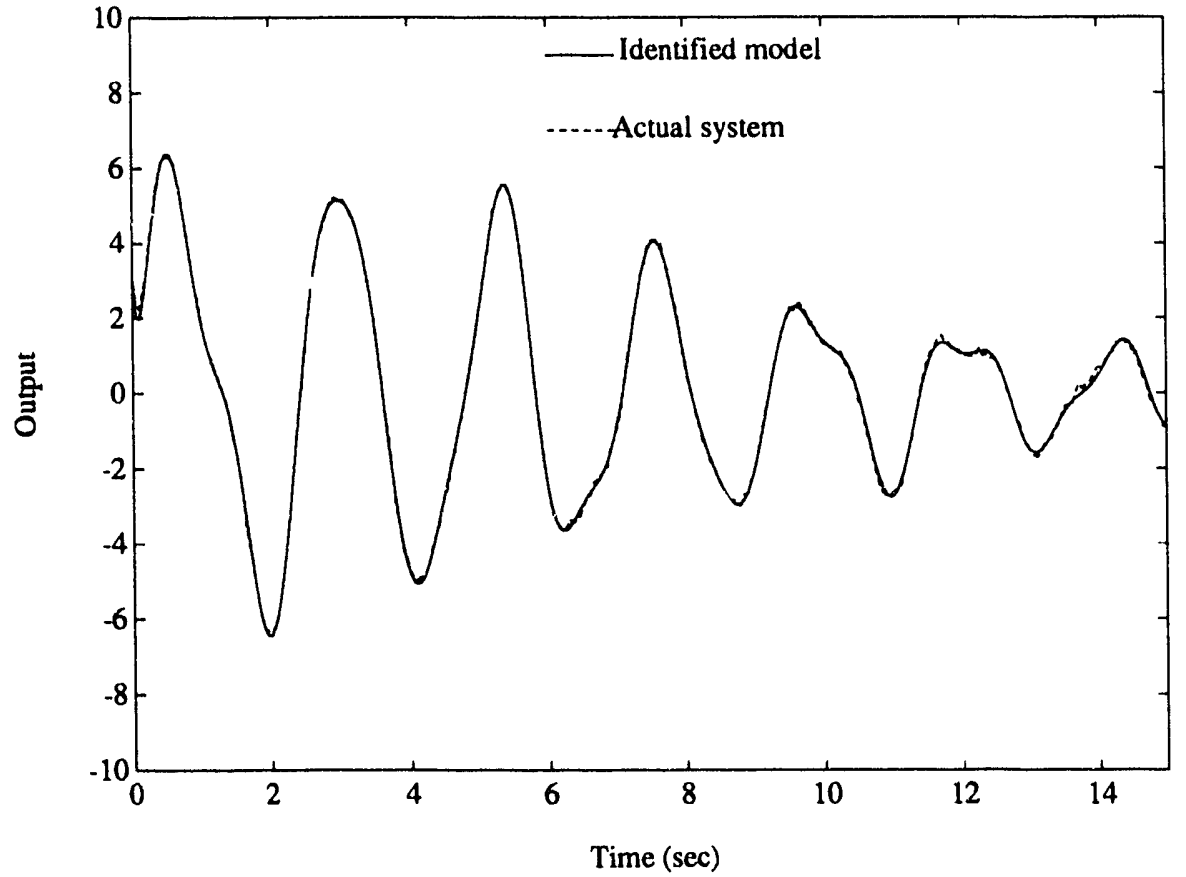

Figure 5.16. Impulse response of the $p=0.9$ fit, Example 5.8. 


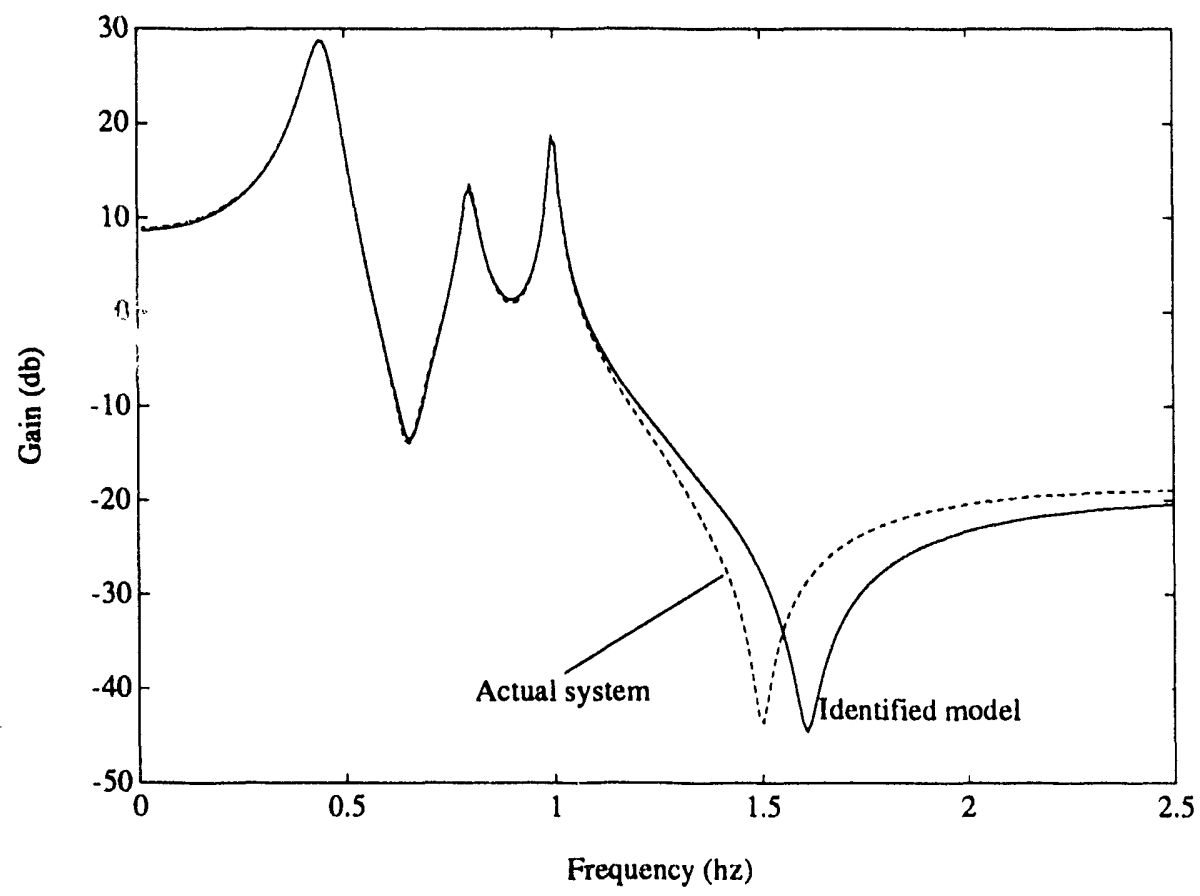

Figure 5.17. Frequency response of the $p=0.9$ fit, Example 5.8.

\section{Example 5.9}

Consider the system

$$
\left.\begin{array}{rl}
G(s)= & \left(\frac{s^{2}+0.2 s+0.1^{2}+(3 \pi)^{2}}{s^{2}+0.1 s+0.05^{2}+(2 \pi)^{2}}\right)\left(\frac{s^{2}+1.2 s+0.6^{2}+(1.8 \pi)^{2}}{s^{2}+0.2 s+0.1^{2}+(1.6 \pi)^{2}}\right) \\
\frac{s^{2}+1.3 s+0.65^{2}+(1.3 \pi)^{2}}{s^{2}+s+0.5^{2}+(0.7 \pi)^{2}}\left(\frac{9.6 \pi}{s+3.2 \pi}\right) \\
\frac{s^{2}+(1.4-p 0.9) s+(0.7-p 0.45)^{2}+(0.9 \pi)^{2}}{s^{2}+0.5 s+0.25^{2}+(0.9 \pi)^{2}}
\end{array}\right)
$$

which is a modified version of test system 1 . As $p$ moves from 0 to 1 , the pole-zero pair near $0.45 \mathrm{hz}$ converge. Figures 5.18 and 5.19 show the response of system (5.6) for $p=0,0.75$, and 0.9. White noise at $30 \mathrm{db}$ SNR is added to the impulse response of, (5.6) and fits are performed for $p=0,0.75$, and 0.9 . Tables 5.6 and 5.7 summarize the identified parameters; closely-spaced terms are highlighted. As the pole-zero pair converge, the identified damping on the pole becomes too large and the estimated residue becomes too large. At $p=0.9$, the estimated damping is nearly double the actual, and the identified residue is nearly three times the actual. Although not shown here, the estimated model very closely matches the time and frequency responses of the actual system despite the error for the closely-spaced terms. 


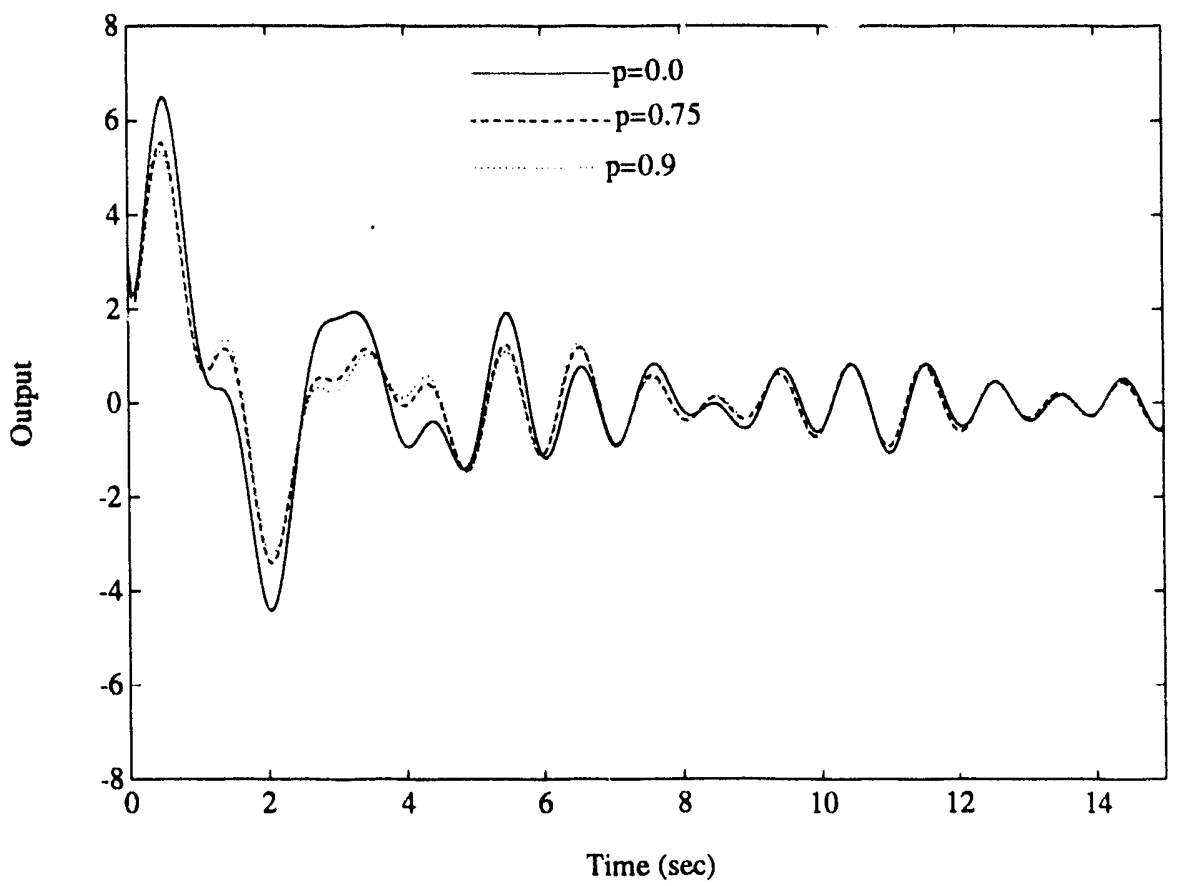

Figure 5.18. Impulse response of system (5.6), Example 5.9.

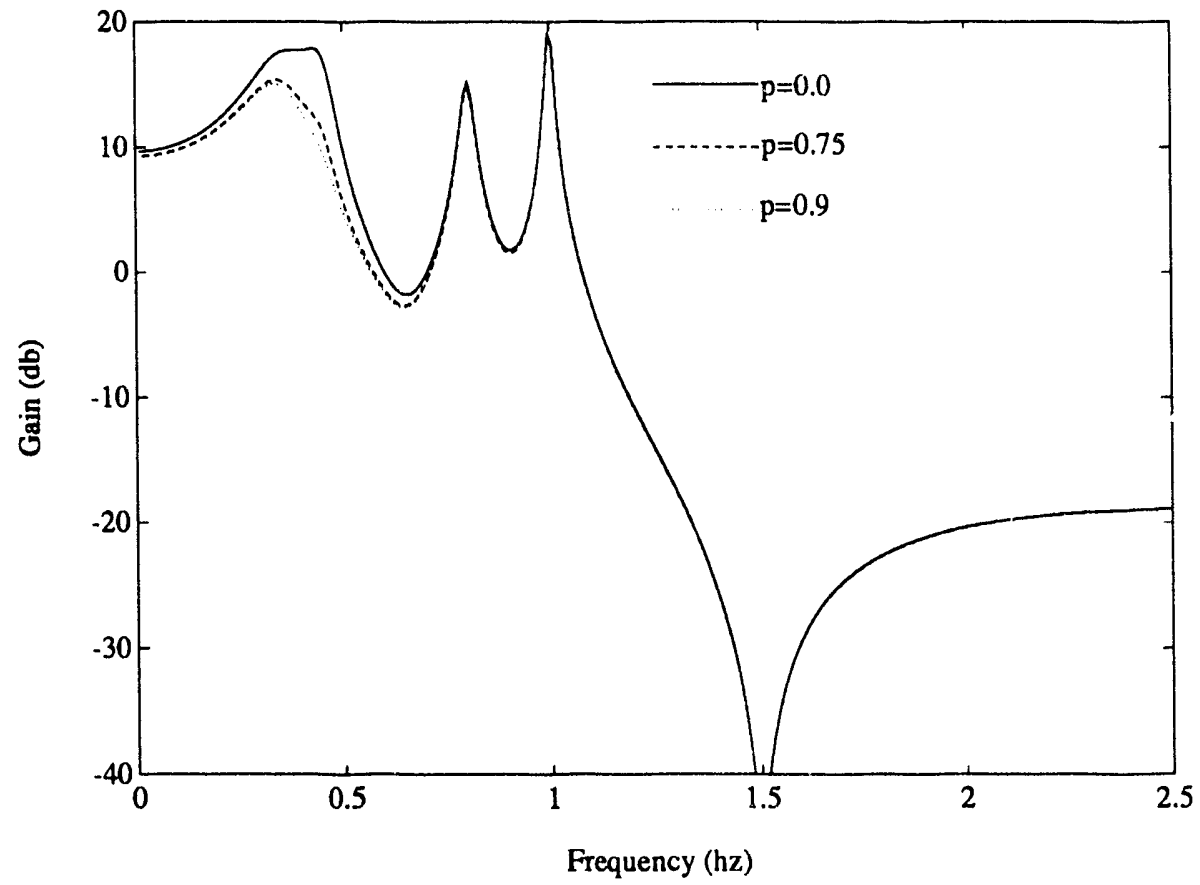

Figure 5.19. Frequency response of system (5.6), Example 5.9. 


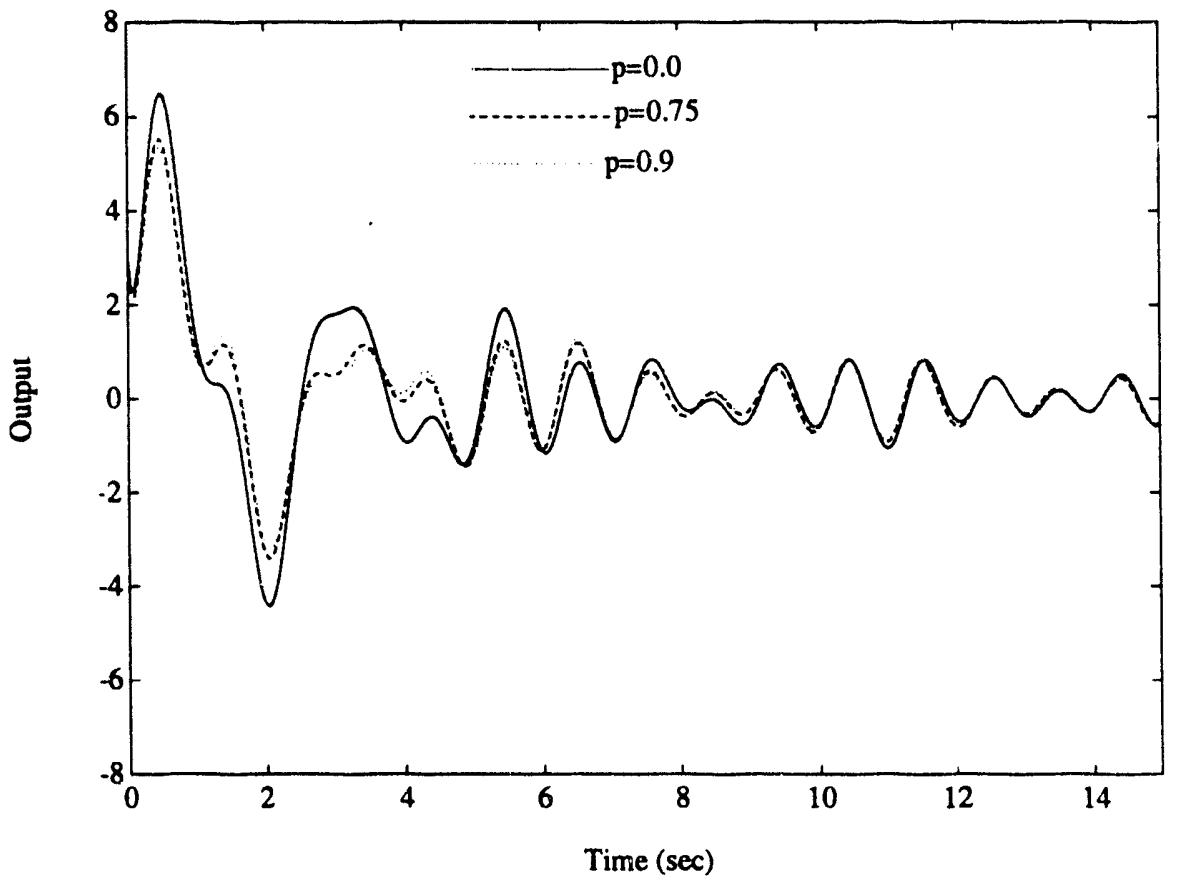

Figure 5.18. Impulse response of system (5.6), Example 5.9.

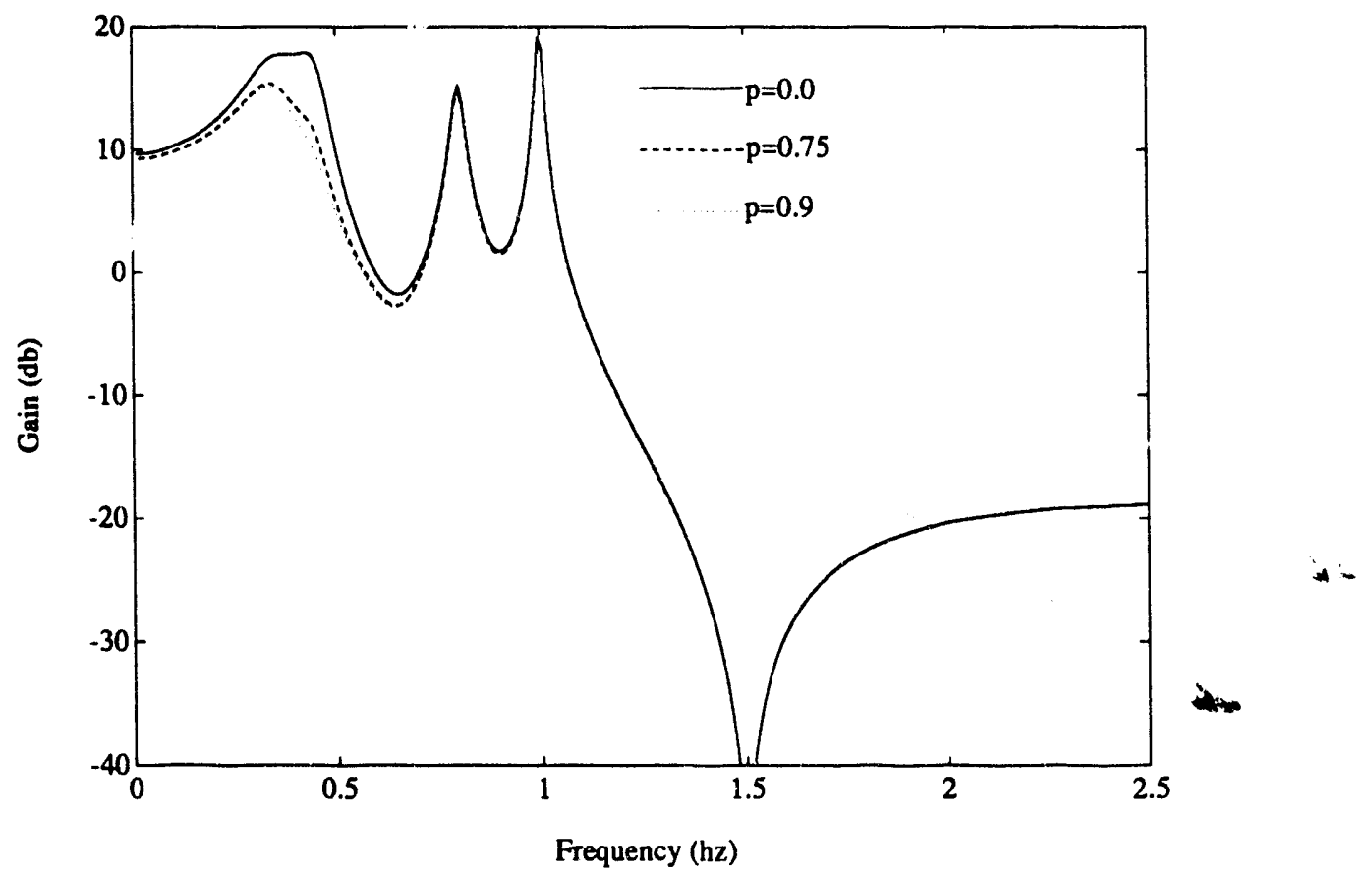

Figure 5.19. Frequency response of system (5.6), Example 5.9. 
Table 5.6. Identified poles for closely-spaced pole-zero pairs, Example 5.9.

\begin{tabular}{||l|l|l|l||}
\hline \multicolumn{1}{|c|}{ Actual } & \multicolumn{1}{|c|}{$\begin{array}{c}\text { Identified } \\
\mathrm{p}=0.0\end{array}$} & \multicolumn{1}{c|}{$\begin{array}{c}\text { Actual } \\
\mathrm{p}=0.75\end{array}$} & \multicolumn{1}{c|}{$\begin{array}{c}\text { Identified } \\
\mathrm{p}=0.9\end{array}$} \\
\hline$-1.6(2 \pi)$ & Not identified & Not Identified & Not Identified \\
\hline$-0.5 \pm j 0.35(2 \pi)$ & $-0.521 \pm j 0.346(2 \pi)$ & $-0.509 \pm j 0.346(2 \pi)$ & $-0.516 \pm j 0.346(2 \pi)$ \\
\hline$-0.25 \pm j 0.45(2 \pi)$ & $-0.273 \pm j 0.449(2 \pi)$ & $-0.325 \pm j 0.449(2 \pi)$ & $-0.475 \pm j 0.461(2 \pi)$ \\
\hline$-0.10 \pm j 0.80(2 \pi)$ & $-0.0997 \pm j 0.799(2 \pi)$ & $-0.101 \pm j 0.799(2 \pi)$ & $-0.101 \pm j 0.799(2 \pi)$ \\
\hline$-0.05 \pm j 1.00(2 \pi)$ & $-0.0525 \pm j 1.00(2 \pi)$ & $-0.0516 \pm j 1.00(2 \pi)$ & $-0.0514 \pm j 1.00(2 \pi)$ \\
\hline
\end{tabular}

Table 5.7. Identified residues for closely-spaced pole-zero pairs, Example 5.9.

\begin{tabular}{|c|c|c|c|c|c|}
\hline $\begin{array}{l}\text { Actual } \\
p=0.0\end{array}$ & $\begin{array}{c}\text { Identified } \\
\mathrm{p}=0.0\end{array}$ & $\begin{array}{c}\text { Actual } \\
p=0.75\end{array}$ & $\begin{array}{c}\text { Identified } \\
\mathrm{p}=0.75\end{array}$ & $\begin{array}{l}\text { Actual } \\
p=0.9\end{array}$ & $\begin{array}{c}\text { Identified } \\
p=0.9\end{array}$ \\
\hline 4.95 & Not Ident. & 4.22 & Not Ident. & 4.28 & Not Ident. \\
\hline $\begin{array}{l}2.55 \\
\angle \mp 57.7^{\circ}\end{array}$ & $\begin{array}{l}2.54 \\
\angle \mp 51.5^{\circ}\end{array}$ & $\begin{array}{l}2.49 \\
\angle \mp 83.8^{\circ}\end{array}$ & $\begin{array}{l}2.39 \\
\angle \mp 79.0^{\circ}\end{array}$ & $\begin{array}{l}2.56 \\
\angle \mp 88.8^{\circ}\end{array}$ & $\begin{array}{l}2.47 \\
\angle \mp 81.7^{\circ}\end{array}$ \\
\hline $\begin{array}{l}1.28 \\
\angle \mp 153^{\circ}\end{array}$ & $\begin{array}{l}1.45 \\
\angle \mp 155^{\circ}\end{array}$ & $\begin{array}{l}0.318 \\
\angle \mp 149^{\circ}\end{array}$ & $\begin{array}{l}0.466 \\
\angle \mp 157^{\circ}\end{array}$ & $\begin{array}{l}0.127 \\
\angle \mp 149^{\circ}\end{array}$ & $\begin{array}{l}0.367 \\
\angle \mp 175^{\circ}\end{array}$ \\
\hline $\begin{array}{l}0.513 \\
\angle \mp 116^{\circ}\end{array}$ & $\begin{array}{l}0.506 \\
\angle \mp 115^{\circ}\end{array}$ & $\begin{array}{l}0.498 \\
\angle \mp 105^{\circ}\end{array}$ & $\begin{array}{l}0.493 \\
\angle \mp 104^{\circ}\end{array}$ & $\begin{array}{l}0.496 \\
\angle \mp 103^{\circ}\end{array}$ & $\begin{array}{l}0.491 \\
\angle \mp 102^{\circ}\end{array}$ \\
\hline $\begin{array}{l}0.483 \\
\angle \pm 178^{\circ}\end{array}$ & $\begin{array}{l}0.491 \\
\angle \pm 177^{\circ}\end{array}$ & $\begin{array}{l}0.476 \\
\angle \mp 175^{\circ}\end{array}$ & $\begin{array}{l}0.481 \\
\angle \mp 176^{\circ}\end{array}$ & $\begin{array}{l}0.475 \\
\angle \mp 173^{\circ}\end{array}$ & $\begin{array}{l}0.480 \\
\angle \mp 174^{\circ}\end{array}$ \\
\hline
\end{tabular}




\subsection{Conclusions and Recommendations}

This report demonstrates the characteristics of using Prony analysis to identify linear dynamic models from system impulse response data. Special attention is paid to systems with several closelyspaced damped modes. For the most part, the characteristics are established by conducting a number of fits under controlled conditions and studying the results of these fits. Proposed future work centers around using the results in this report to further advance the Prony analysis software.

\subsection{Conclusions}

The main thrust of the work presented here has centered around investigating the characteristics of Prony analysis for fitting to the impulse response data of oscillatory systems. From this investigation, several "rules of thumb" can be developed for making Prony analysis an effective analysis tool.

The following is a list of general guidelines that can be used in fitting linear systems to damped impulse response data using Prony analysis. It is important to note that these are only guidelines based on experience, not hard rules.

- In sampling the system data, use a sample period at least double the Nyquist rate but not more than 10 times the Nyquist rate.

- In choosing the length of the data record, several factors must be considered: 1 ) at least one full cycle of the minimum-frequency oscillatory mode should be included (more data is preferred); 2) if data is collected in a noisy environment, an excessively long data record should be avoided (this is somewhat a judgment, but the record should be short enough to keep the SNR above $20 \mathrm{db}$ ); 3) if a double-precision root finder is employed, the number of data points should not exceed 250 (a safer limit is 100); and 4) for a quadprecision root finder, the limits are 500 and 200.

- In most cases, one should not expect to accurately identify stable real poles with a magnitude greater than $1 /(3 T)$, where $T$ is the sample period.

- In fitting to the data, the following algorithm choices are likely to give the best results: 1) use the covariance method in the forward direction to formulate the LP problem; 2) there are presently no obvious differences between the L.P solution algorithms available in PRSPAK, but SVD currently seems to be a safe first choice; 3 ) if possible, use the quadprecision root finder, and 4) make the order of the Vandermonde problem the same as the LP problem.

- In choosing the order of the LP fit, use $N / 3$ to $N / 2$, as long as it does not exceed 50 for the double-precision root finder or 100 for the quad.

- Accurate fits are likely if the SNR is $20 \mathrm{db}$ or greater. If the noise is highly colored, poor fits are likely for poles and residues in the bandwidth of the colored noise.

- If closely-spaced poles are identified, especially with similar imaginary terms, the error 
bounds for these terms are likely to be large, even if the fit is accurate graphically. This may be an indication to combine the modes as one composite term.

\subsection{Recommended Future Work}

The work presented here was conducted as part of a first phase in the development of a Power System Identification (PSI) Toolbox. Several extensions of this work are proposed with the objective being the further development of Prony analysis programs and methods for eventual inclusion in the toolbox.

A practical vision of the analysis programs in the Toolbox is that they exist at two levels. The first level would be similar to the commonly used LINPACK (Dongarra et al. 1979) or Numerical Recipes (Press et al. 1990) subroutines. Each routine would cinsist of standard FORTRAN coding with non-platform-dependent input options and no graphical options. The second level would consist of a few "highly intelligent" programs developed for specific platforms with appropriate graphical and user-friendly interfaces. The core analysis routines for the second level would consist of the routines from the first.

Using the first level of routines, analysis programs could be incorporated into other analysis programs (for example, data collection software). For Prony analysis, the subroutine PRSPAK would be the primary analysis routine. Other possible routines would be ORDER and TRANSFUN employed in IPRONYID (see Appendix C for current versions of these subroutines), and many of the routines called by subroutine PRGEN in SIGPAKZ.

Proposed future work for the next phase focuses on forming the level one routines for the Toolbox and further developing the Prony analysis codes. Future proposed work includes 1) updating and overhauling the current version of subroutine PRSPAK; 2 ) implementing a formal revision system for all routines in SIGPAKZ and IPRONYID for developing modifications; 3) incorporating the transfer function identification routine TRANSFUN into SIGPAKZ; 3) incorporating "local intelligence" into SIGPAKZ and IPRONYID based on results presented in this report; 4) further investigating new options for solving the Prony analysis problem and processing results; and 5) investigating the use of combined time-domain (Prony analysis) and frequency-domain methods to identify transfer functions.

\subsubsection{Overhaul PRSPAK}

As discussed in Section 3, the current version of PRSPAK is a subroutine developed from the program DTRANSIENTS. In its current form, it contains coding developed at the University of Colorado at Boulder and the Bonneville Power Administration. As it is used in IPRONYID and SIGPAKZ, several options are not used and a few errors exists in the routine. The proposed overhaul primarily involves making PRSPAK a clean level-one subroutine. The objective is make PRSPAK an efficient routine that is as close to standard FORTRAN as possible. Modifications will be made to 1) correct errors; 2) remove all platform-dependent graphing options; 3) remove all timing calculations; 4) incorporate new interactive and batch options; and 5) do a general variable clean up. The goals are to make PRSPAK a flexible subroutine that can easily be incorporated into many different standalone programs, both custom and commercial, and to make implementation of new algorithms as simple as possible. 
To date, the following errors have been encountered in the version of PRSPAK obtained from BPA:

- There is no check to make sure NSIZE is not exceeded.

- In solving the LP problem using standard QR or TLS, PRSPAK jumps to statement 390, an IF block checking lor an error in the fast QR algorithm. In some circumstances it is possible that PRSPAK could use the fast QR algorithm when the TLS or standard $Q R$ is desired. To fix this change, the GOTO 390 statements after the TLS and standard QR to GOTO 400.

- Fix comments in the code (e.g., the comment for the section of code that searches for the strongest mode inside the interval [FTRIML,FTRIMH] states that the interval outside [FTRIML,FTRIMH] is searched). Also, the comment on line 812 should read NNHAT in place of NNFIT, and several main header comments are outdated.

- There is no return statement in PRSPAK (it should be after the section of code starting at statement 8800 .

The current version of PRSPAK has interactive graphing options that are supported on only specific platforms. Also, some of the routines called by PRSPAK (such as LSTQR) have plotting options. These plotting options are memory- and time-consuming; also, because the plotting options are platform-dependent, porting the code to different platforms is very difficult. For example, when PRSPAK was ported to a SUN workstation at PNL, the plotting options had to be disabled. Therefore, it is proposed that all plotting options be removed from PRSPAK. Using the interactive and batching options proposed below, all plotting options could still be performed, but outside PRSPAK.

The current BPA version of PRSPAK has an interactive option and a batch option. With the batch option, key variables are output to a data file. With PRSPAK2 version 1.1.2.1 described in Section 3, a second batch option is added to PRSPAK. This option is the same as the first batch option, but no variables are written to an output file. This allows PRSPAK to be a standalone subroutine with all inputting and outputting performed in the subroutine call.

To make PRSPAK as generic as possible, it is proposed that new interactive and batching options be developed. Basically the same interactive option currently used in PRSPAK will be employed. All inputting is performed in subroutines INENTER and LGENTER. Plotting and special read/write options can be incorporated into subroutines INENTER and LGENTER. Future versions of INENTER and LGENTER could range from platform-independent generic read routines to user-friendly platform dependent routines. The proposed batching option is similar to the current form, but with all outputting to data files removed. In place of the outputting, extensive use will be made of the array MRETR (flags for Prony return mode) to indicate the status of the different Prony analysis steps. Then all outputting to data files can be done outside PRSPAK.

The current version of PRSPAK uses the array MSEC to track the CPU timing of different calculations. Because CPU time calculation is platform-dependent, this option is not directly portable. Also, with the speed of modern platforms, the computation time of the algorithms employed in PRSPAK is relatively trivial. For these reasons, it is proposed that the CPU time calculation options 
be removed from PRSPAK. If one desires to track computation time, many of the modem compilers and debugging programs allow one to do so.

The final steps in overhauling PRSPAK will be to develop a document that describes the routine in detail. This will later become a chapter in an extensive manual discussing all the level one subroutines.

\subsubsection{Implement Revision Control System}

Currently, no revision control system is employed with the BPA-based Prony analysis programs. In the work conducted for this report, an initial revision system was implemented using the public domain Revision Control System (RCS) (Tichy 1985). With this system, modifications and version numbers are automatically tracked. Several options are possible with RCS for customizing to the users' needs.

Because UC is currently developing new Prony-related algorithms that may be implemented into the current version of SIGPAKZ and IPRONYID, it is proposed that a set coding standard be adopted for developing new routines. These standards are to be developed in conjunction with UC and BPA and based on an industry standard (such as IEEE). Such a system will ensure timely, efficient, and accurate incorporation of new algorithms into the analysis software.

It is proposed that PNL be responsible for assigning and tracking version numbers. Modifications to PRSPAK will be conducted in conjunction with UC to ensure that fitting algorithms are properly incorporated. The general idea is to have the routine PRSPAK and the main calling routine of the UC program TRANSIENTS be parallel routines in that new options incorporated into TRANSIENTS can easily be implemented into PRSPAK, or vice versa.

\subsubsection{Incorporate Transfer Function Identification in SIGPAKZ}

The current version of SIGPAKZ allows transfer function terms to be calculated only from a single pulse input. Several extensions have been developed that allow much more general inputs to be used. A subroutine called TRANFUN has been developed at PNL for use with IPRONYID that allows the identification of transfer function terms for a general input involving a series of square-wave pulses. It is proposed that this subroutine be incorporated into SIGPAKZ and be called from the subroutine PRSGEN. One possible way to do this is to incorporate the calculations in TRANFUN into the subroutines PRSMOD1 and 2.

\subsubsection{Develop "Local intelligence" in SIGPAKZ and IPRONYID}

The results from the tests discussed in this report will be used to develop "intelligent" decision and calculation options into PRSGEN and PRSPAK. This includes data-dependent defaulting and possibly iterative caivilation options.

\subsubsection{Investigate New Options}

Areas that merit further investigation before being implemented into SIGPAKZ include model ordering and reduction methods; composite modes; error bounds on parameters; eigenanalysis for LP 
polynomial rooting, as well as other rooting algorithms; use of LAPACK (Anderson et al. 1992) routines in place of LINPACK ones; and investigation of alternative LP solution methods discussed in the literature review. Investigation of many of these new options will be coordinated with or conducted at $\mathrm{CU}$ based on BPA and PNL discretion.

The current version of SIGPAKZ outputs models in the order of lowest frequency to highest frequency. No effort is given to re-order the terms from most important to least important, and it is often impossible to distinguish between terms that are true system modes and modes due to noise effects. The model order reduction method recently developed at PNL has proven to be very valuable in solving this problem (a modified version of the ordering algorithm is contained in IPRONYID). Only one case seems to cause sensitivities, that is the one of very closely-spaced poles. It is proposed that this algorithm be tested and, if feasible, implemented in SIGPAKZ.

The composite mode method developed at UC is used to identify models with repeated roots. The method primarily involves combining modes that are closely spaced. Error bound algorithms for identified parameters are also being developed at UC using Cramer-Rao bound theory (McWhorter and Scharf 1991). It is proposed that these methods be tested for use in PRSPAK. This testing will be conducted in close association with UC.

Currently, PRSPAK has only one algorithm for rooting the LP polynomial. Although this algorithm has proven to give good results in many cases, other options are available. These include eigenanalysis. It is proposed that other rooting methods, including eigenaralysis, be compared and tested.

LAPACK (Anderson et al. 1992) is the next generation of LINPACK developed by the National Science Foundation. It represents significant improvements in computational efficiency and numerical robustness. It is proposed (and highly recommended) that LAPACK QR and SVD routines be implemented in place of the current LINPACK ones used in PRSPAK.

The last new option that merits investigation involves solution methods for the LP problems contained in Kay and Marple (1987); Marple (1980); Minami (1986); and Cruz (1986). Currently, these methods are not contained in PRSPAK, but show merit based on the literature. 


\subsection{References}

Akiake, H. 1969. "Fitting Autoregressive Models for Prediction." Mathematical Review 21:243-247.

Anderson, E., Z. Bai, C. Bischof, J. Demmel, J. Dongarra, J. Du Croz, A. Greenbaum, S. Hammarling, A. Mckenney, S. Ostrouchov, and D. Sorensen. 1992. LAPACK User's Guide. Society for Industrial and Applied Mathematics, Philadelphia.

Barone, P. 1988. "Some Practical Remarks on the Extended Prony's Method of Spectrum Analysis." Proceedings of the IEEE 76(3):284-285.

Barone, P., L. Guidoni, E. Massaro, and V. Viti. 1987. "Prony-Burg Method for NMR Spectral Analysis." Journal of Magnetic Resonance 73:23-33.

Cruz, J. R. 1986. "Spectral Analysis Using the QD Algorithm." In Proceedings of the IEEE International Conference on Acoustics, Speech, and Signal Processing, vol. 2, pp. 1345-1348. April 1986, Tokyo, Japan. Institute of Electrical and Electronics Engineers, Piscataway, New Jersey.

Demeure, C. J. 1989. "Fast QR Factorization of Vandermonde Matrices." Journal of Linear Algebra and Its Applications 124:165-194.

Demeure, C. J., and L. L. Scharf. 1990. "Sliding Windows and Lattice Algorithms for Computing QR Factors in the Least Squares Theory of Linear Prediction." IEEE Transactions on Acoustics, Speech, and Signal Processing ASSP-38(4):721-725.

Friddell, T. H., J. A. Ritcey, and D. Haynor. 1989. "Data Pre-conditioning for Improved Performance of the Prony Method." In Proceedings of the 23rd Asilomar Conference on Signals, Systems, and Computers, pp. 360-364. October 1989, Pacific Grove, California. Institute of Electrical and Electronics Engineers, Piscataway, New Jersey.

Hauer, J. F., C. J. Demeure, and L. L. Scharf. 1990. "Initial Results in Prony Analysis of Power System Response Signals." IEEE Transactions on Power Systems. 5(1):80-89.

Hauer, J. F. 1991. "The Use of Prony Analysis to Determine Modal Content and Equivalent Models for Measured Power System Response." In IEEE Symposium on Eigenanalysis and Frequency Domain Methods for System Dynamic Performance, pp. 105-115. Publication 90TH0292-3-PWR, Institute of Electrical and Electronics Engineers, Piscataway, New Jersey.

Hauer, J. F., and C. W. Taylor. 1990. Feedback Control and Frequency Domain analysis of Large Electric Power Systems, Vol. 1: Seminar Workbook. Carson Taylor Seminars, Portland, Oregon.

Dongarra, J. J., C. B. Moler, J. R. Bunch, and G. W. Stewart. 1979. LINPACK User's Guide. Society for Industrial and Applied Mathematics, Philadelphia.

Hildebrand, F. B. 1956. Introduction to Numerical Analysis. McGraw Hill, New York. 
Hocking, R. R., and R. N. Leslie. 1967. "Selection of the Best Subset in Regression Analysis." Technometrics 9(4):531-540.

Hoxha, F., C. Lambert, and J. Noailles. 1988. "Experiments in Concurrently Computing Polynomial Roots." In Proceedings of the 4th European Signal Processing Conference, pp. 659-662. September 1988, Grenoble, France. Elsevier Science Publishers B. V., North-Holland.

Jenkins, M. A. 1975. "Algorithm 493, Zeros of a Real Polynomial." ACM Transactions on Mathematical Software 1(2):178-189.

Jenkins, M. A., and J. F. Traub. 1970. "A Three Stage Algorithm for Real Polynomials Using Quadratic Iteration." SIAM Journal on Numerical Analysis 7(4):545-566.

Kay, S. M., and S. L. Marple. 1981. "Spectrum Analysis -- A Modern Perspective." Proceedings of the IEEE 69:1380-1419.

Kumaresan, R., and D. W. Tufts. 1982. "Estimating the Parameters of Exponentially Damped Sinusoids and Pole-Zero Modeling in Noise." IEEE Trans. on Acoustics, Speech, and Signal Processing ASsP-30(6):833-840.

Kumaresan, R., D. W. Tufts, and L. L. Scharf. 1984. "A Prony Method for Noisy Daia: Choosing the Signal Componenis and Selecting the Order in Exponential Signal Models." Proceedings of the IEEE 72(2):230-233.

Lambert, C., and F. Castanie. 1988. "An Adaptive Prony Algorithm." In Proceedings of the 4th European Signal Processing Conference, pp. 571-574. September 1988, Grenoble, France. Elsevier Science Publishers B. V., North-Holland.

Lancaster, P., and M. Tismenetsky. 1985. The Theory of Matrices. Academic Press, New York.

Madsen, K. 1973. "A Root-Finding Algorithm Based on Newton's Method." BIT 13:71-75.

Marple, S. L. 1989. "A Tutorial Overview of Modern Spectral Estimation." In Proceedings of the International Conference on Acoustics, Speech, and Signal Processing, pp. 2152-2157. Glasgow, Scotland. Institute of Electrical and Electronics Engineers, Piscataway, New Jersey.

Marple, L. 1980. "A N̦ew Autoregressive Spectrum Analysis Algorithm." IEEE Trans. on Acoustics, Speech, and Signal Processing ASSP-28(4):441-454.

McWhorter, T., and L. L. Scharf. 1991. Cramer-Rao Bounds for Modal Analysis. Technical Report DSP-504, Department of Electrical Engineering, University of Colorado, Boulder.

Minami, K., N. Schlumberger, and S. Kawata. 1986. "Prony's Method Based on Eigenanalysis and Overdetermined System Approach." In Proceedings of the IEEE International Conference on Acoustics, Speech, and Signal Processing (ICASSP), vol. 2, pp.1393-1396. April 1986, Tokyo, Japan. Institute of Electrical and Electronics Engineers, Piscataway, New Jersey. 
Mueller, D. E. 1956. "A Method for Solving Algebraic Equations Using an Automatic Computer." Mathematical Tables Aids Computation 10:208-215.

Pierre, D. A., J. R. Smith, D. J. Trudnowski, and J. W. Pierre. 1992. "General Formulation of a Prony Based Method for Simultaneous Identification of Transfer Functions and Initial Conditions." In Proceedings of the 31st IEEE Conference on Decision and Control, (To be published in December 1992). Institute of Electrical and Electronics Engineers, Piscataway, New Jersey.

Pierre, D. A., D. J. Trudnowski, and J. F. Hauer. 1992. "Identifying Linear Reduced-Order Models for Systems with Arbitrary Initial Conditions Using Prony Signal Analysis." IEEE Transactions on Automatic Control 37(6):831-835.

Poggio, A. J., M. L. Van Blaricum, E. K. Miller, and R. Mittra. 1978. "Evaluation of a Processing Technique for Transient Data." IEEE Trans. on Antennas and Propagation AP-26(1):165-173.

Porat, B., and B. Friedlander. 1986. "A Modification of the Kumaresan-Tufts Method for Estimating Rational Impulse Responses." IEEE Trans. on Acoustics, Speech, and Signal Processing ASSP-34(5):1336-1338.

Press, W. H., B. P. Flannery, S. A. Teukolsky, and W. T. Vetterling. 1990. Numerical Recipes, The Art of Scientific Computing. Cambridge University Press, New York.

Prony, B. de. 1795. "Essai Expérimental et Analytique: sur les Lois de la Dilatabilité de Fluides Élastiques et Sur Celles de la Force Expansive de la Vapeur de l'Eau et de la Vapeur de l'Alcool, à Différentes Températures." Journal de l'École Polytechnique 1(2):24-76.

Rao, D.V.B. 1986a. "An Explanation of the Limitation Observed in the Kumaresan-Prony Algorithm." IEEE Trans. on Acoustics, Speech, and Signal Processing ASSP-34(5):1338-1340.

Rao, D.V.B. 1986b. "An Analysis of the Kumaresan-Prony Method." In Proceedings of the 20th Asilomar Conference on Signals, Systems, and Computers, pp. 467-471. November 1986, Pacific Grove, California. Institute of Electrical and Electronics Engineers, Piscataway, New Jersey.

Rautenberg, T., D. J. Gingras, and J. F. Bohme. 1988. "Comparison of Some Real Coefficients Polynomial Zero-Finder Methods with Respect to Applications in Spectrum Estimation." In Proceedings of the 4th European Signal Processing Conference, pp. 187-190. September 1988, Grenoble, France. Elsevier Science Publishers B. V., North-Holland.

Rutishauser, H. 1957. Der Q-D Algorithmus. Birkhauser, Basel.

Scharf, L. L. 1991. Statistical Signal Processing: Detection, Estimation, and Time Series Analysis. Addison-Wesley Publishing Company, New York.

Steiglitz, K., and L. E. McBride. 1965. "A Technique for the Identification of Linear Systems." IEEE Trans. on Automatic Control AC-10:461-464. 
Steiglitz, K. 1977. "On the Simultaneous Estimation of Poles and Zeros in Speech Analysis." IEEE Trans. on Acoustics, Speech, and Signal Processing ASSP-25(3):229-234.

Tichy, W. F. 1985. "RCS -- A System for Version Control." Software -- Practice \& Experience 15(7):637-654.

Trudnowski, D. J., J. R. Smith, T. A. Short, and D. A. Pierre. 1991. "An Application of Prony Methods in PSS Design for Multimachine Systems." IEEE Transactions on Power Systems 6(2):118126.

Tufts, D. W., and R. Kumaresan. 1982a. "Singular Value Decomposition and Improved Frequency Estimation Using Linear Prediction." IEEE Trans. on Acoustics, Speech, and Signal Processing ASSP-30(4):671-675.

Tufts, D. W., and R. Kumaresan. 1982b. "Estimation of Frequencies of Multiple Sinusoids: Making Linear Prediction Perform Like Maximum Likelihood." Proceedings of the IEEE 70(9):975-989.

Van Blaricum, M. L., and R. Mittra. 1975. "A Technique for Extracting the Poles and Residues of a System Directly from Its Transient Response." IEEE Trans. on Antennas and Propagation AP-23(6):777-781.

Van Blaricum, M. L., and R. Mittra. 1978. "Problems and Solutions Associated with Prony's Method for Processing Transient Data." IEEE Trans. on Antennas and Propagation AP-26(1):174-182.

West, B. J. 1990. "The Noise in Natural Phenomena." American Scientist 7:40-45. 


\section{Appendix A}

\section{PRSPAK2 Version 1.1.2.1}




\section{Appendix A}

\section{PRSPAK2 Version 1.1.2.1}

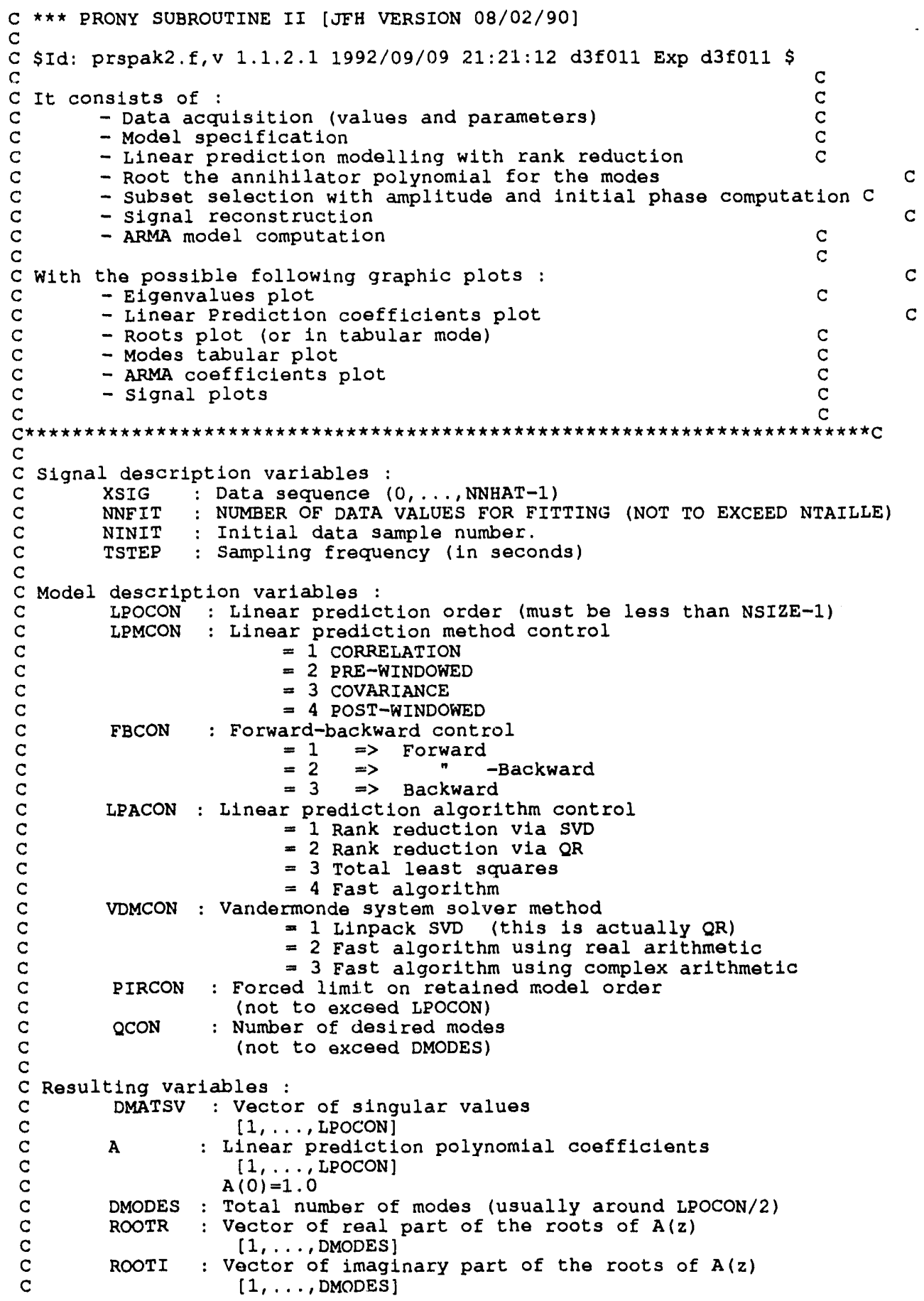




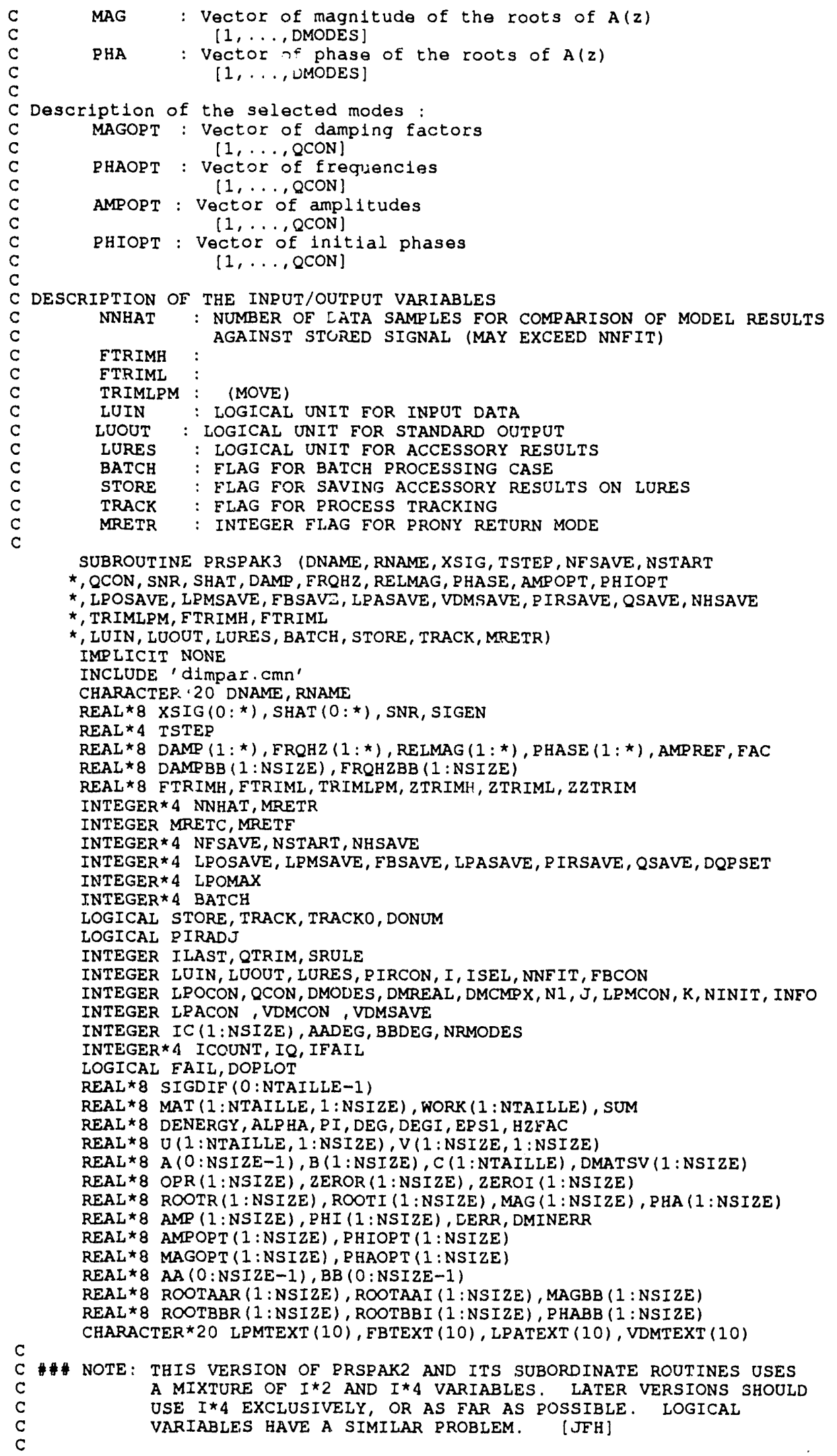




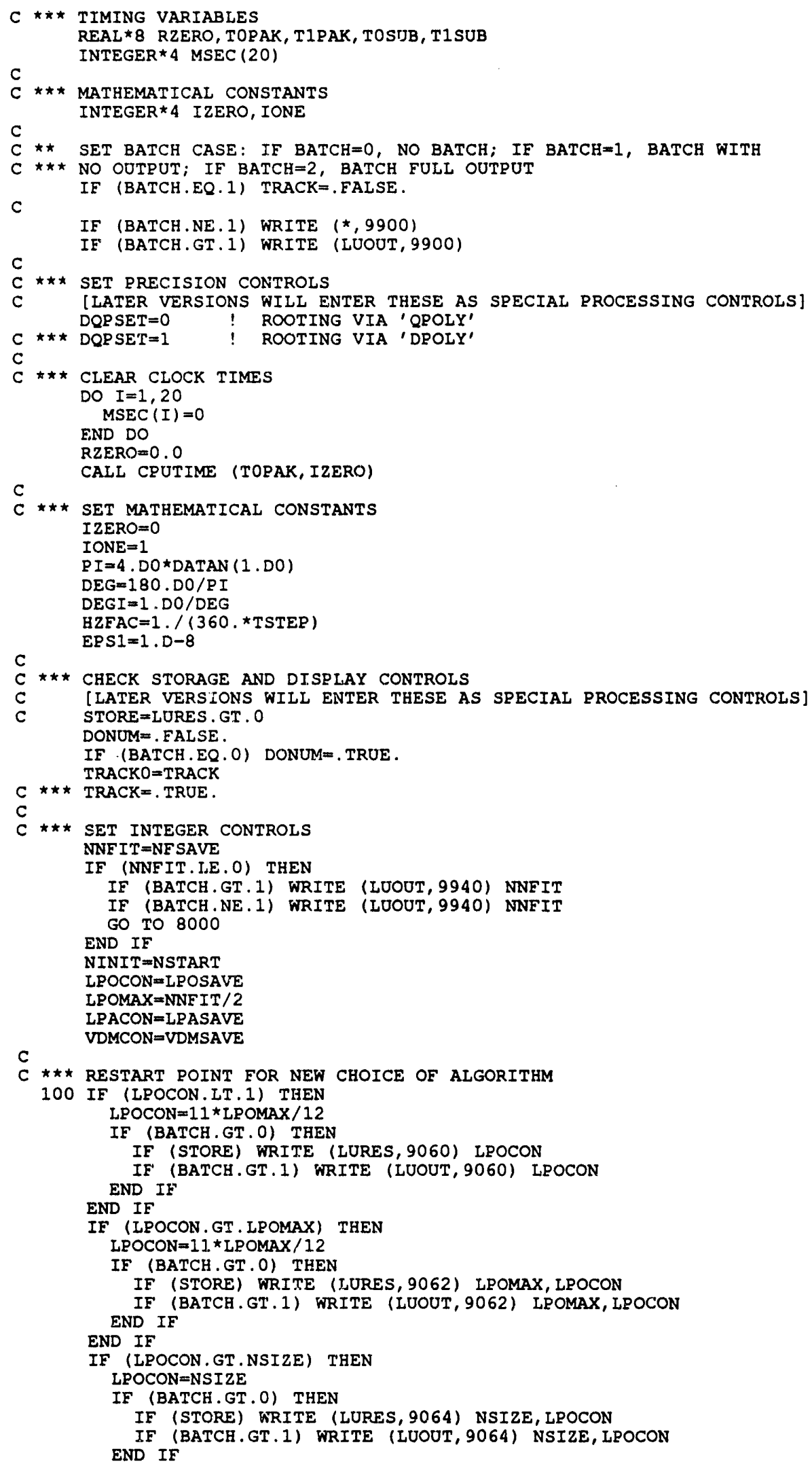




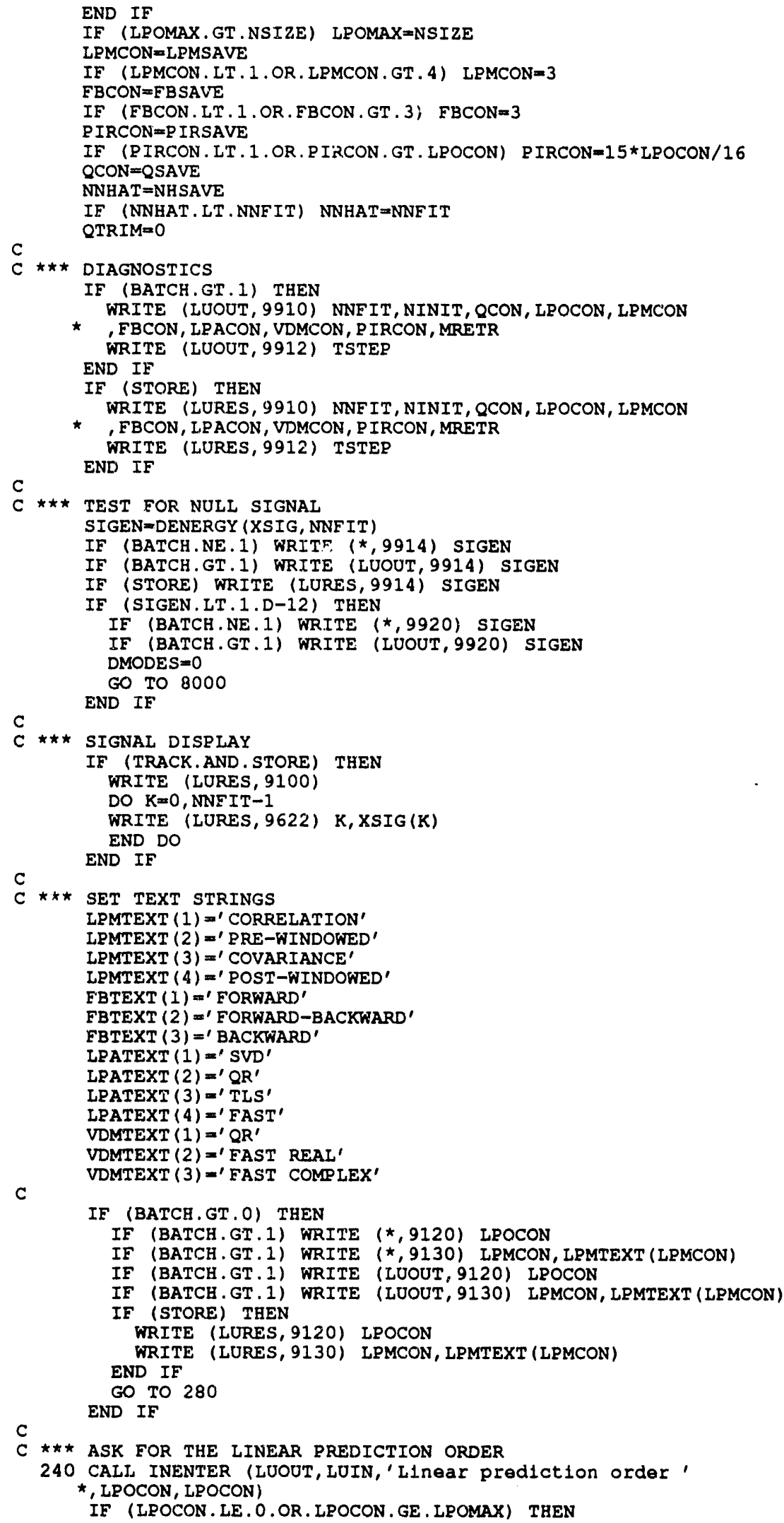




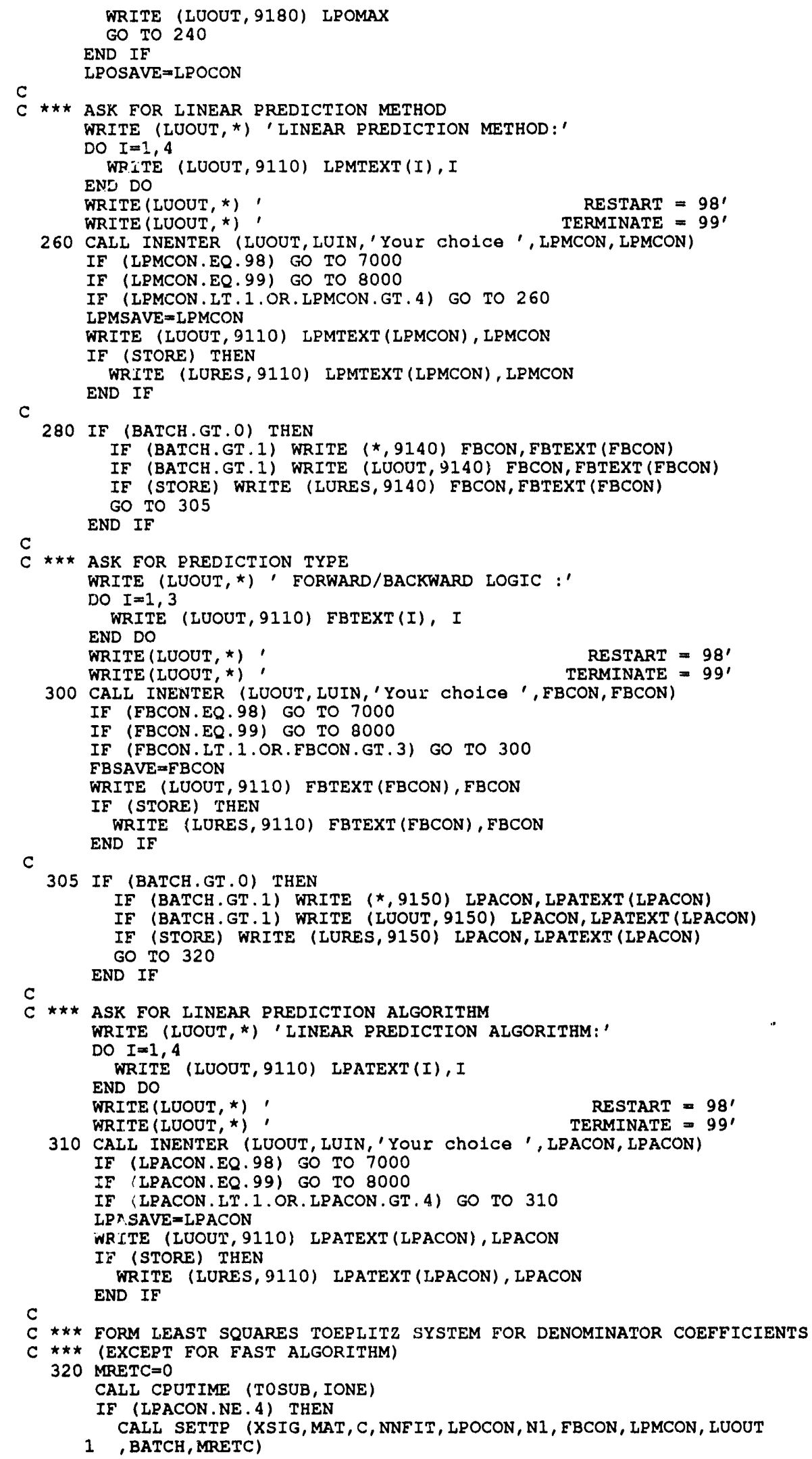




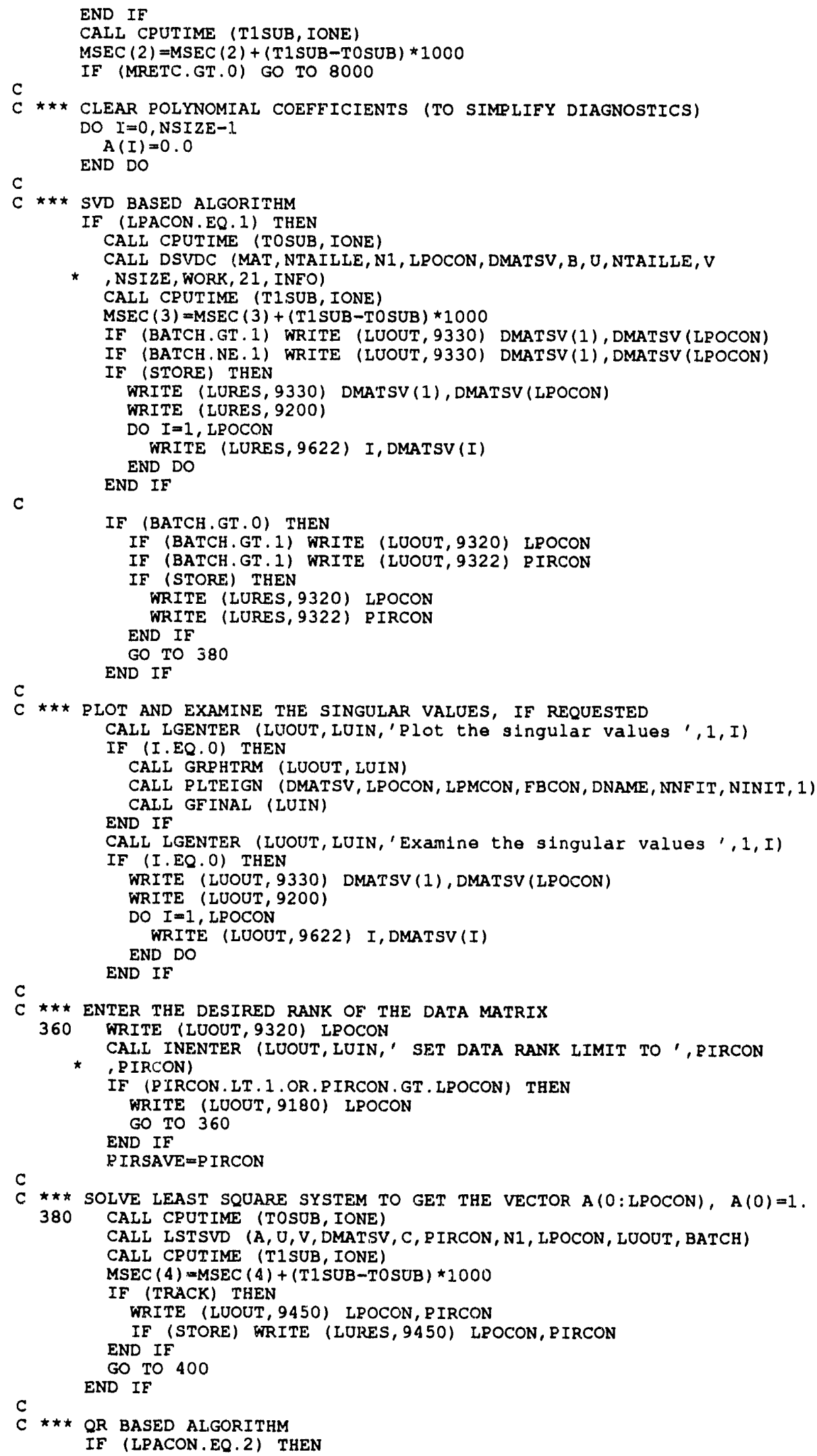


CALL CPUTIME (TOSUB, IONE)

CALL LSTQR (MAT, C, A, N1, LPOCON, LPMCON, FBCON, DNAME, NNFIT

1 , NINIT, PIRCON, LUOUT, LUIN, BATCH)

CALL CPUTIME (T1SUB, IONE)

$\operatorname{MSEC}(4)=\operatorname{MSEC}(4)+(T 1$ SUB - TOSUB $) \star 1000$

GO TO 400

END IF

C

Total Least Squares solution

IF (LPACON.EQ.3) THEN

CALL CPUTIME (TOSUB, IONE)

CALL TLS (MAT, C, A, N1, LPOCON

CALL CPUTIME (TISUB, IONE)

$\operatorname{MSEC}(4)=\operatorname{MSEC}(4)+($ T1SUB $-T O S U B) * 1000$

GO TO 400

END IF

c

$\star \star \star \star$ FAST LEAST SQUARES ALGORITHM

IF (LPACON.EQ.4) THEN

CALL CPUTIME (TOSUB, IONE)

IF (FBCON.EO.1) THEN

IF (LPMCON.EQ.1) CALL LSCOR (XSIG, NNFIT, A, LPOCON, MRETF)

IF (LPMCON.EQ.2) CALL LSPRE (XSIG, NNFIT, A, LPOCON, 0, MRETF)

1 TRIMLPM, MRETF)

, TRIMLPM, MRETF)

ELSE IF (FBCON.EQ.3) THEN

IF (LPMCON.EQ.1) CALL LSCOR (XSIG, NNFIT, A, LPOCON, MRETF)

IF (LPMCON.EQ.2) CALL LSPRE (XSIG, NNFIT, A, LPOCON, 1, MRETF) (LPMCON EO 3) CALL LSCOV (XSIG, NNFIT, A, LPOCON, IONE , TRIMLPM, MRETF)

IF (LPMCON.EQ.4) CALL LSPST (XSIG, NNEIT, A, LPOCON, 1, MRETF)

ELSE IF (FBCON.EQ.2) THEN

IF (LPMCON.EQ.1) CALL LSCOR (XSIG, NNFIT, A, LPOCON, MRETF)

IF (LPMCON.EQ.2) CALL LSPREFB (XSIG, NNFIT, A, LPOCON, MRETE)

IF (LPMCON.EQ.3) CALL LSCOVFB (XSIG, NNFIT, A, LPOCON, MRETF)

IF (LPMCON.EQ.4) CALI LSPSTFB (XSIG, NNFIT, A, LPOCON, MRETF)

END IF

CALL CPUTIME (TISUB, IONE)

$\operatorname{MSEC}(4)=\operatorname{MSEC}(4)+($ T1 SUB - TOSUB $) * 1000$

GO TO 390

END IF

IF (BATCH.NE.1) WRITE $(*, *)$ 'LPACON TYPE NOT RECOGNIZED' IF (BATCH.GT.1) WRITE (LUOUT, *) 'LPACON TYPE NOT RECOGNIZED'

C

$C \star \star \star$ TEST FAST SOLUTION

390 IF (MRETF. LT.0) THEN

LPACON=1

IF (BATCH.NE.1) WRITE $(*, 9960)$ MRETF, LPACON

IF (BATCH.GT.1) WRITE (LUOUT, 9960) MRETF, LPACON

GO TO 100

C

END IF

$C \star \star \star$ DIAGNOSTIC DISPLAY OF DENOMINATOR $z$-COEFFICIENTS

400 IF (TRACK. OR. STORE) THEN

IF (TRACK) WRITE (LUCUT, 9451)

IF (STORE) WRITE (LURES, 9451)

DO $I=0$, LPOCON

IF (TRACK) WRITE (LOOUT, 9622) I, A (I)

IF (STORE) WRITE (LURES, 9622) I, A(I) END DO

END IF

C

$\star \star \star$
ROOT DENOMINATOR POLYNOMIAL FOR EREQUENCIES AND DAMPING FACTORS
DO $I=0$, LPOCON

OPR $(I+1)=A(L P O C O N-I) \quad$ ! NOTE REVERSAL

END DO

IF (DQPSET.EQ.0) THEN

CALI CPUTIME (TOSUB, IONE)

CALL QPOLYRT (OPR, LPOCON, ZEROR, ZEROI, IFAIL)

CALL CPUTIME (T1SUB, IONE)

$\operatorname{MSEC}(5)=\operatorname{MSEC}(5)+($ T1SUB - TOSUB $) \star 1000$

IF (IFAIL.NE.0) THEN

IF (BATCH.GT.1) WRITE(LUOUT, *) 'QPOLY FAILURE =', IFAIL

1, 'FOR DENOMINATOR'

IF (STOKE) WRITE (LUOUT, *) 'QPOLY FAILURE $=\prime$, IFAIL 


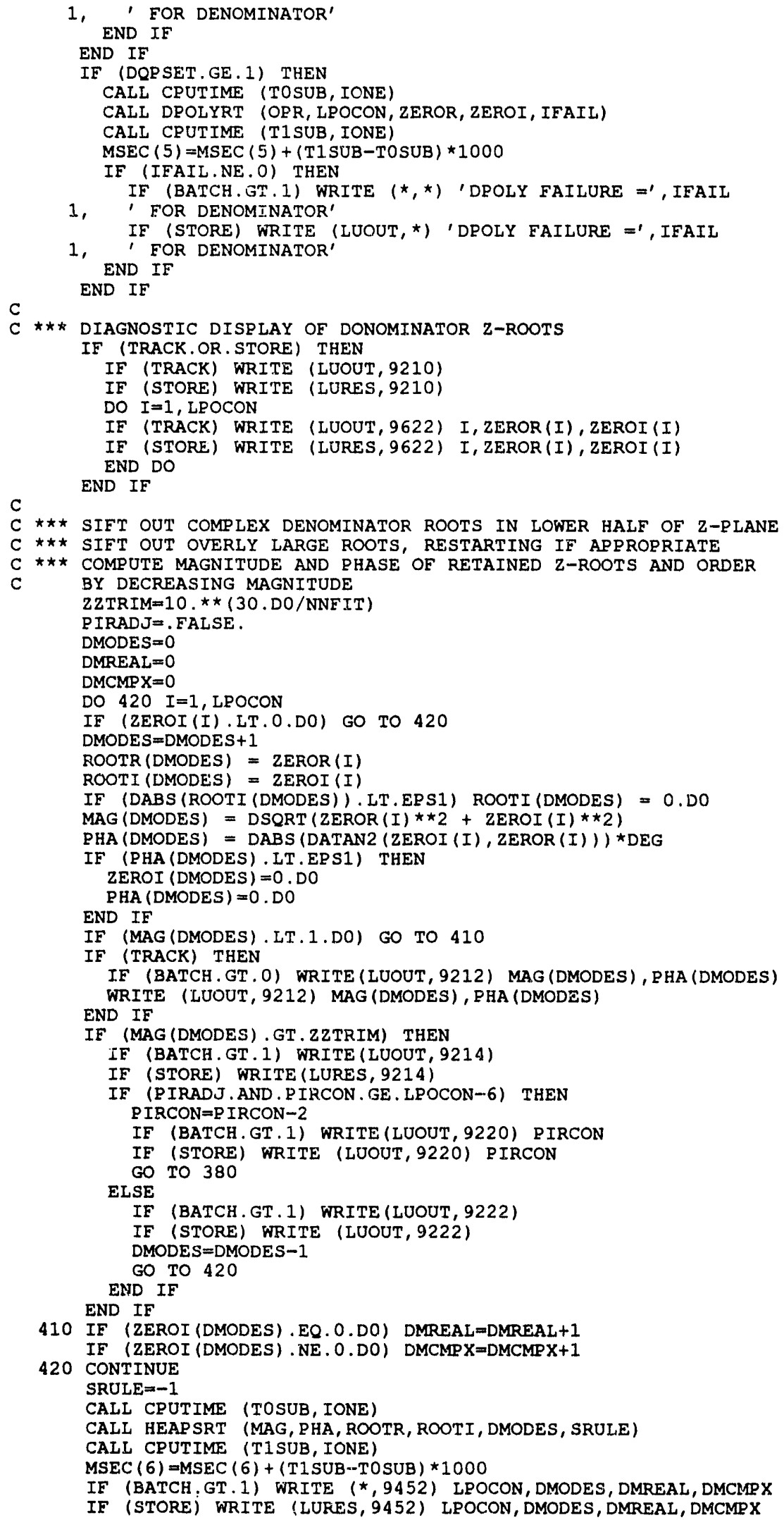




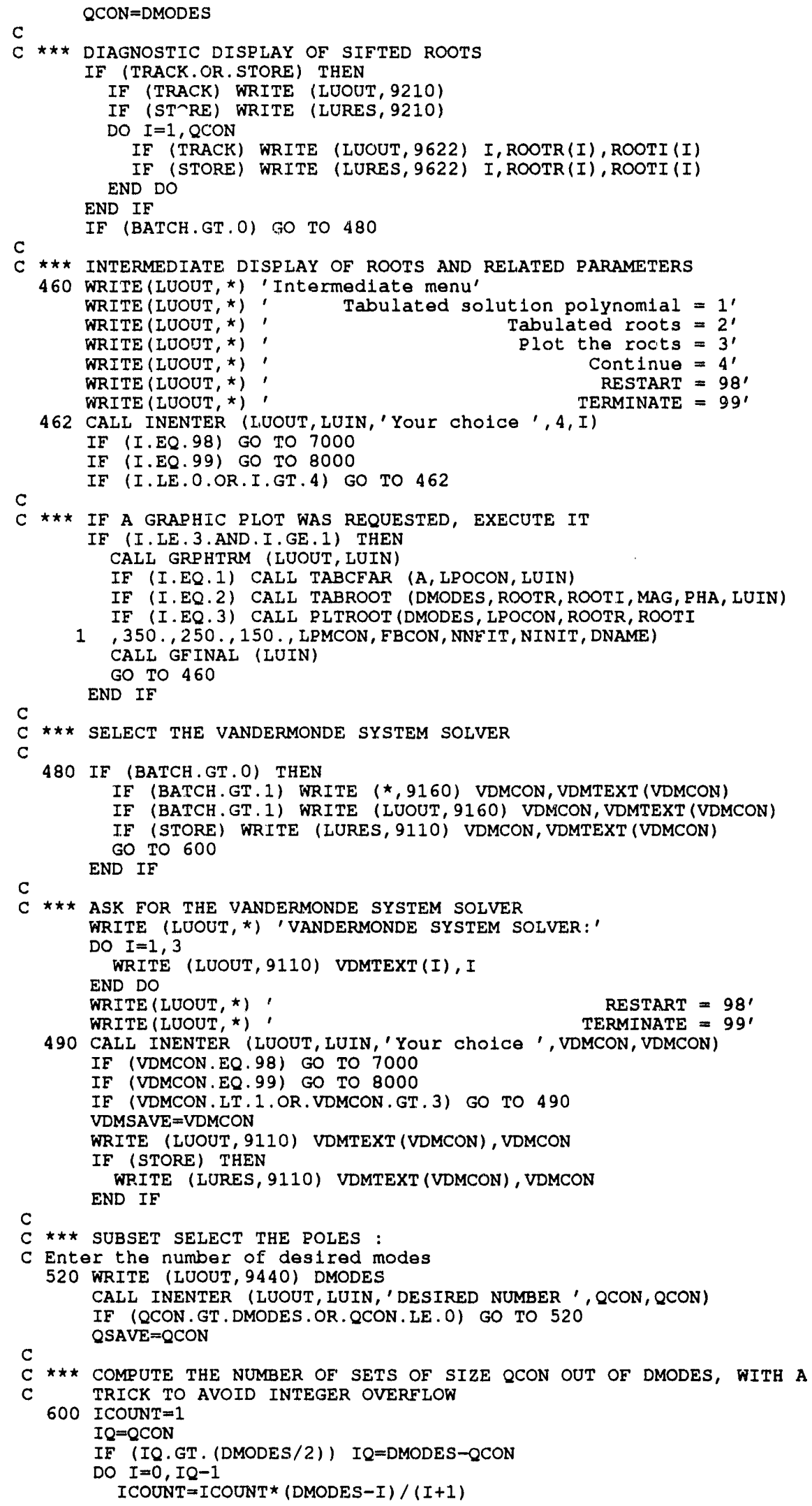




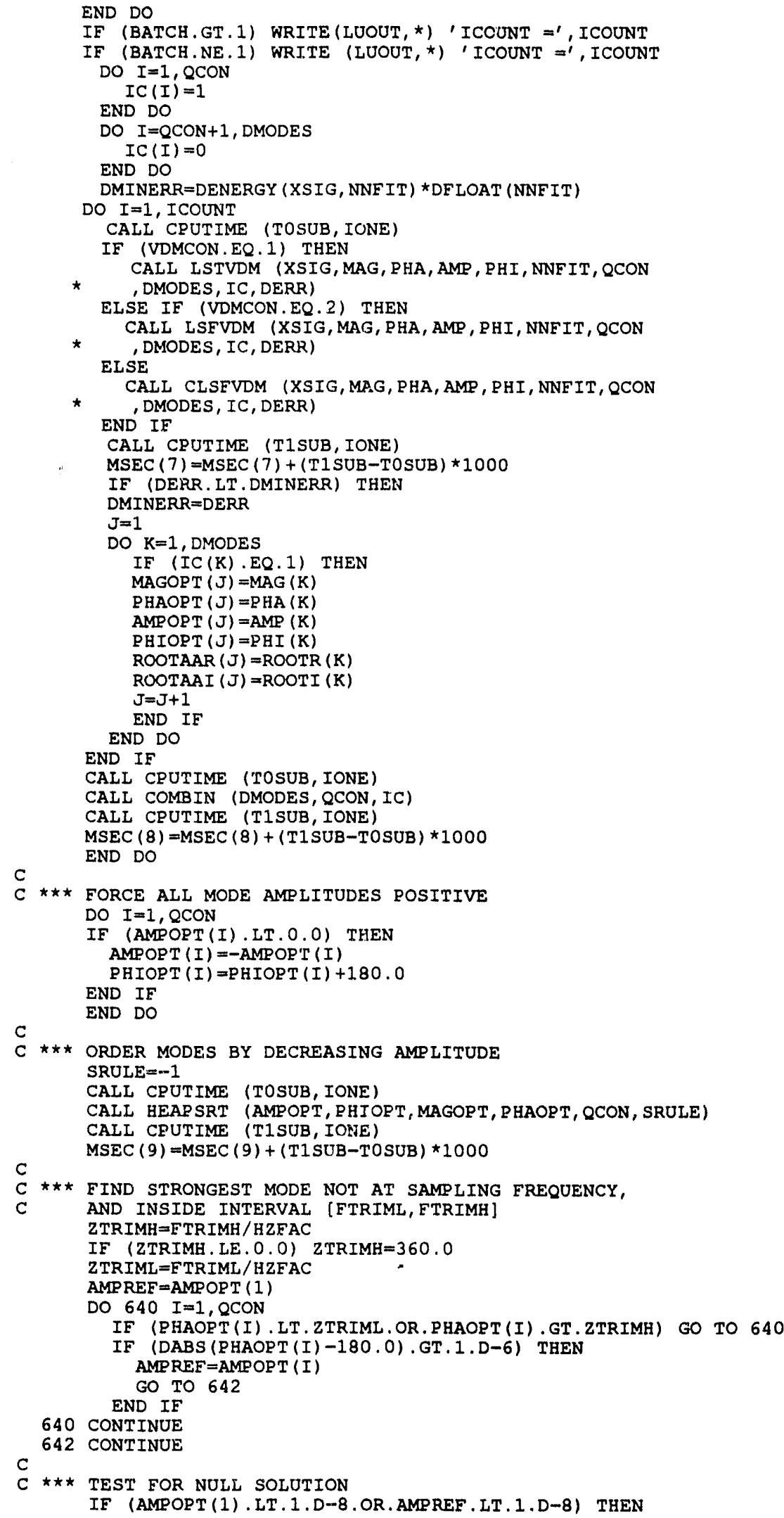




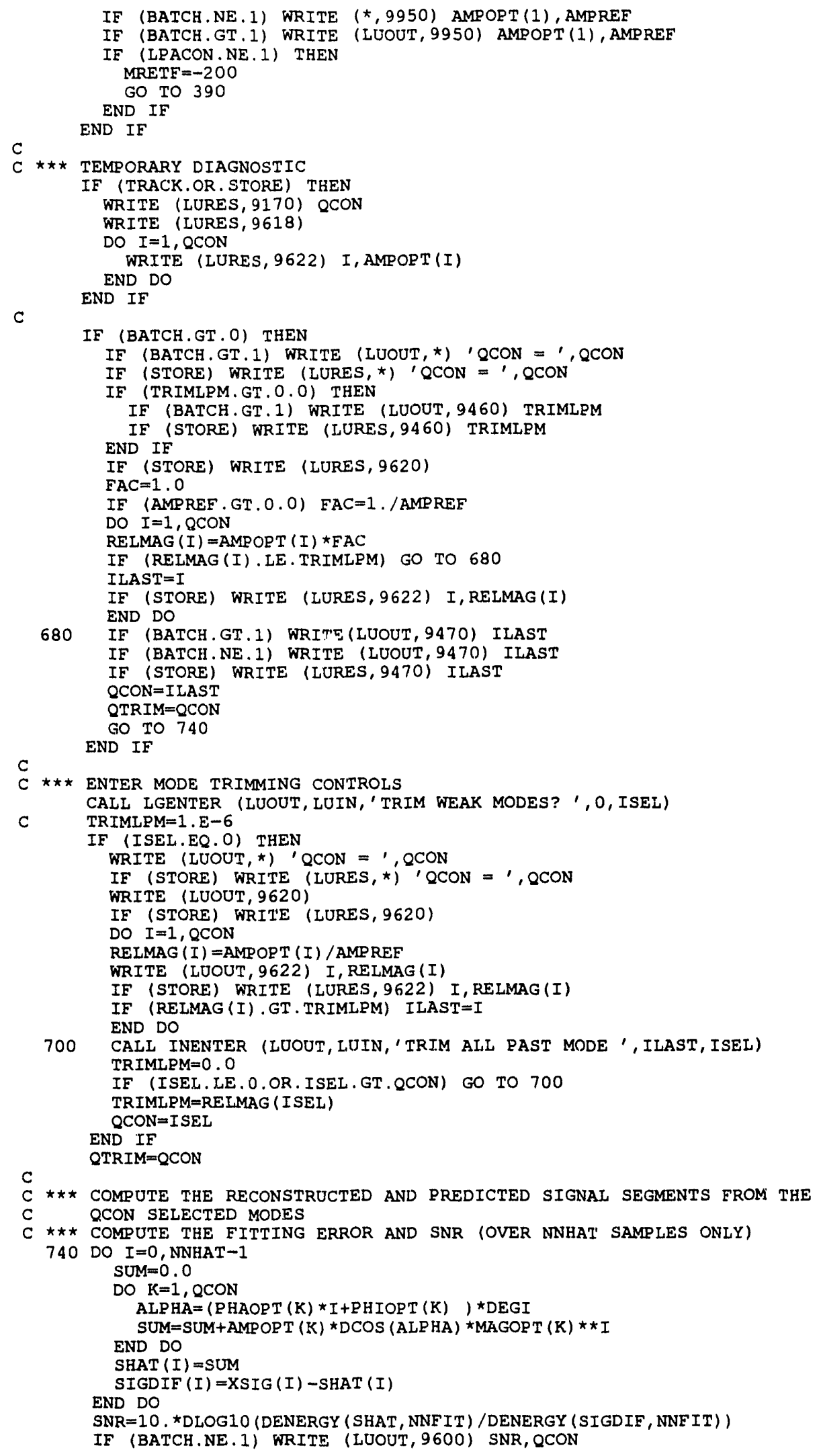




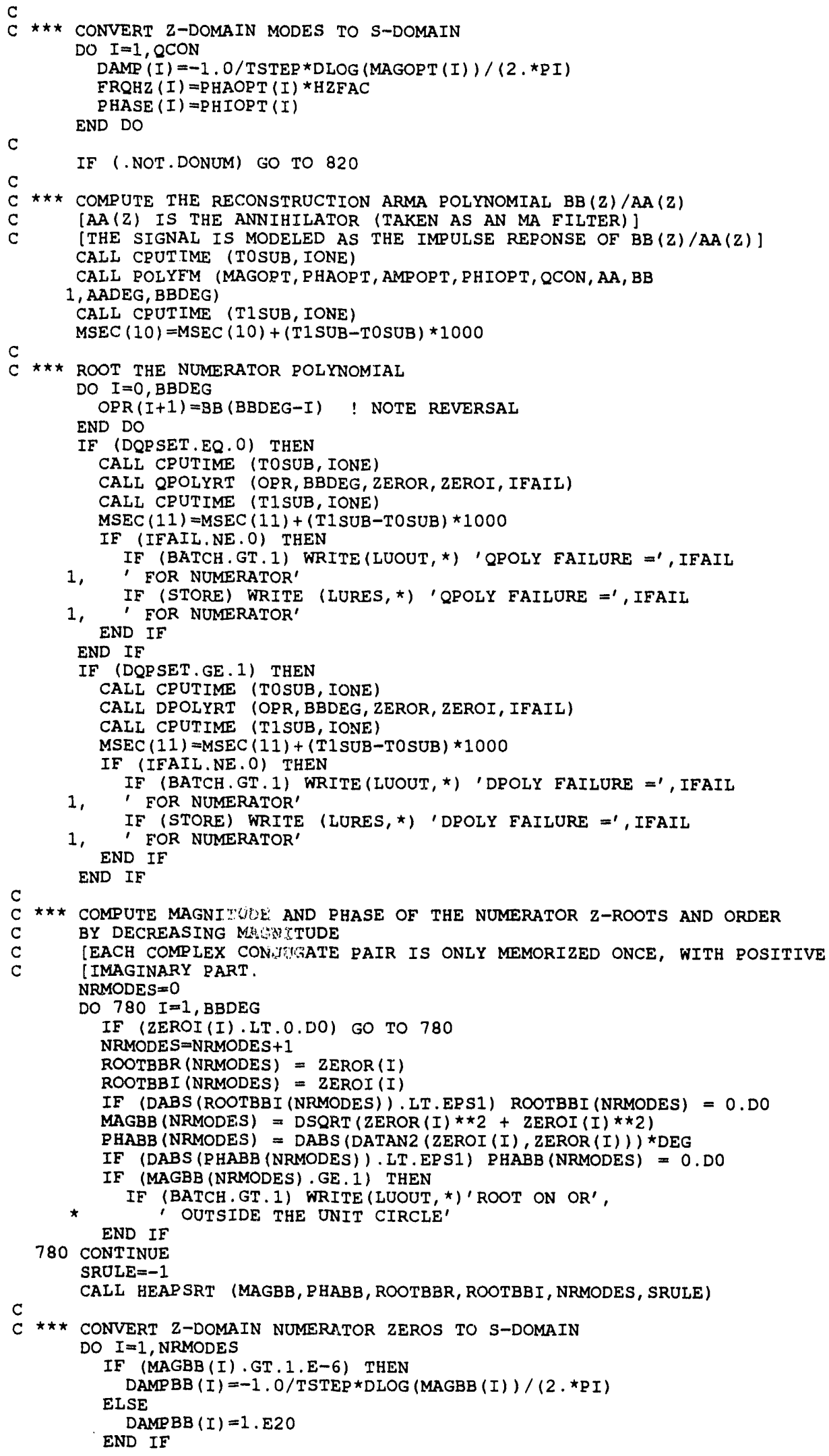




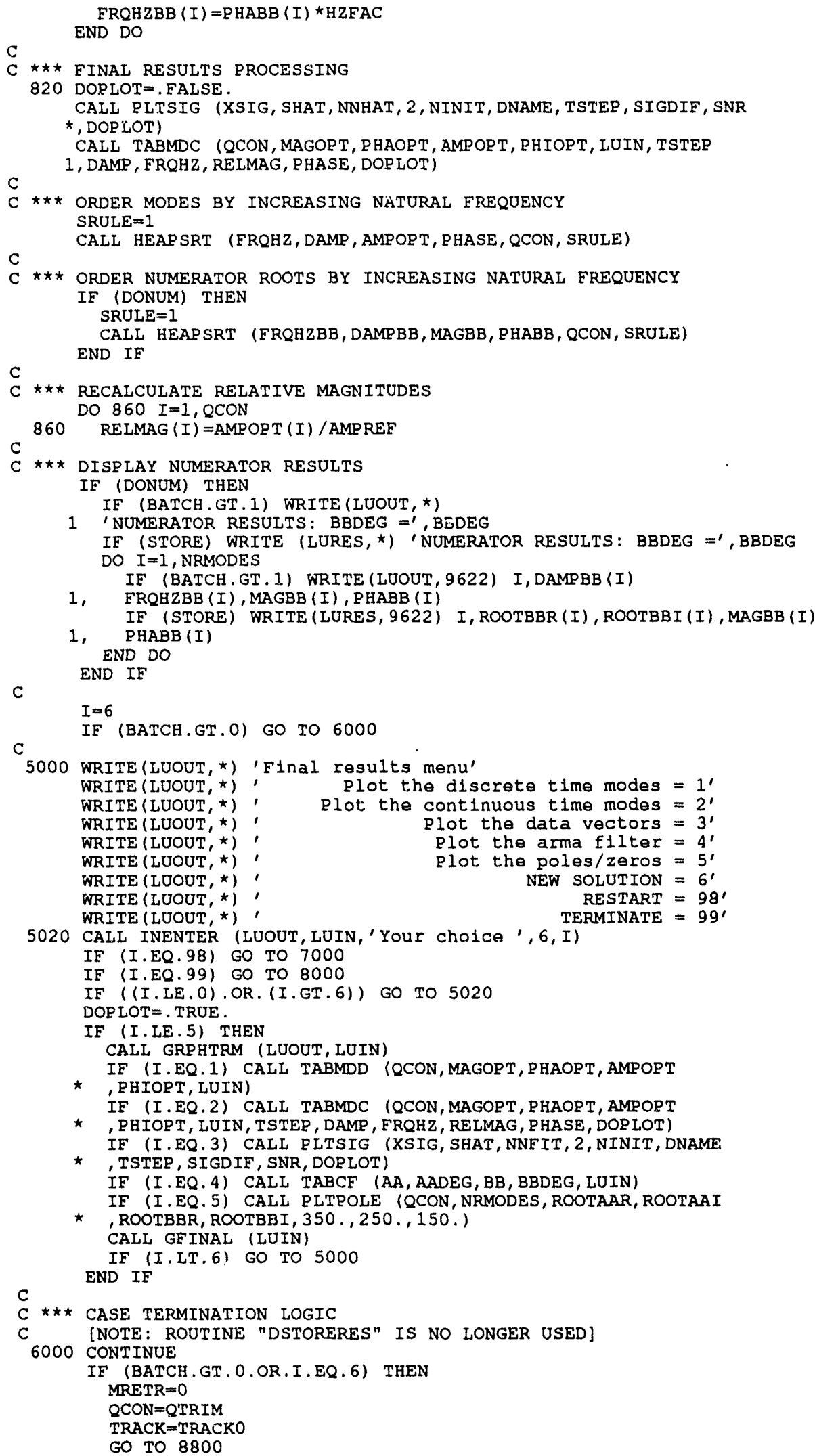




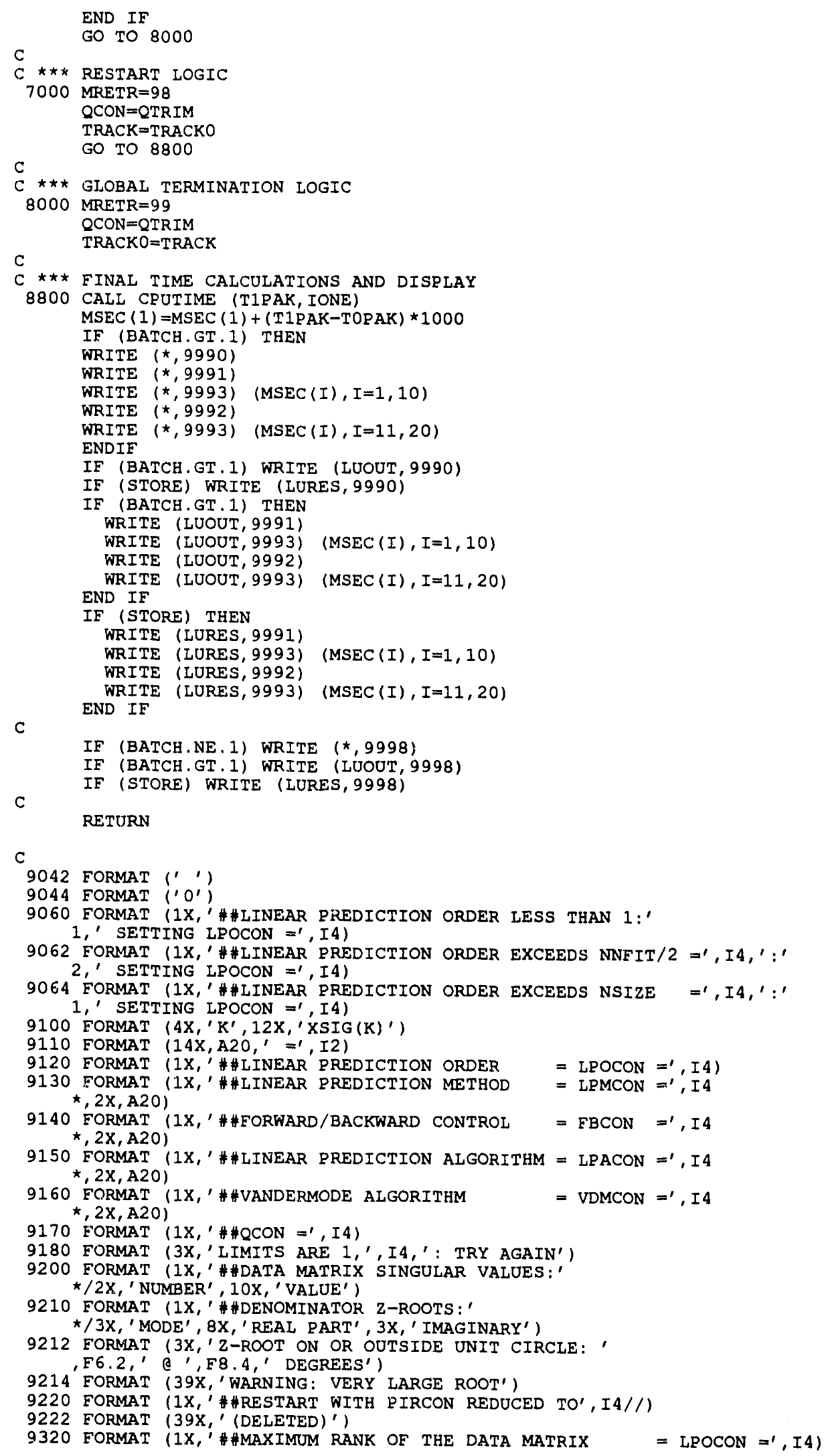




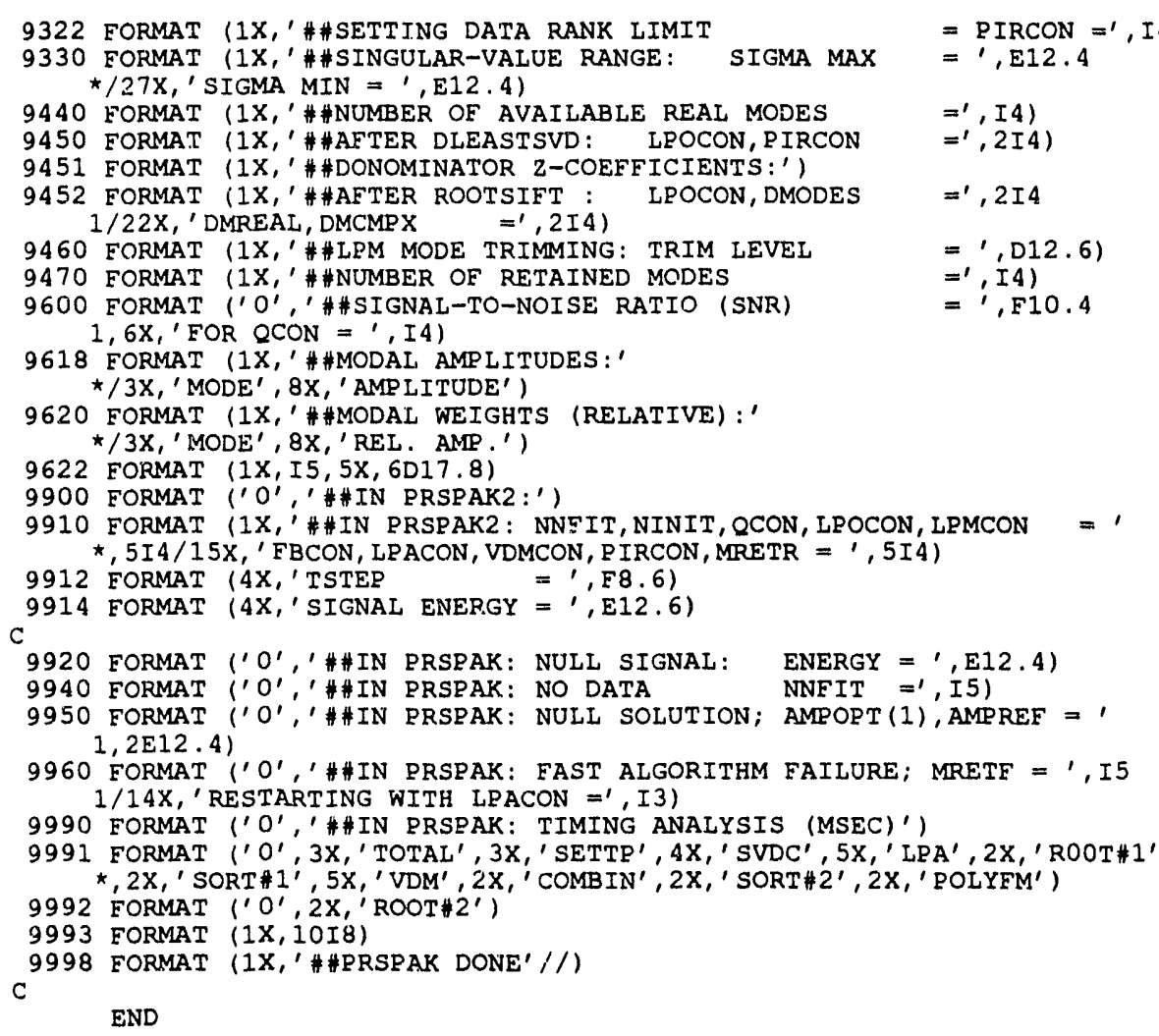


Appendix B

Section 5 SIGPAKZ Input Files 


\section{Appendix B}

\section{Section 5 SIGPAKZ Input Files}

\section{Example 4.1}

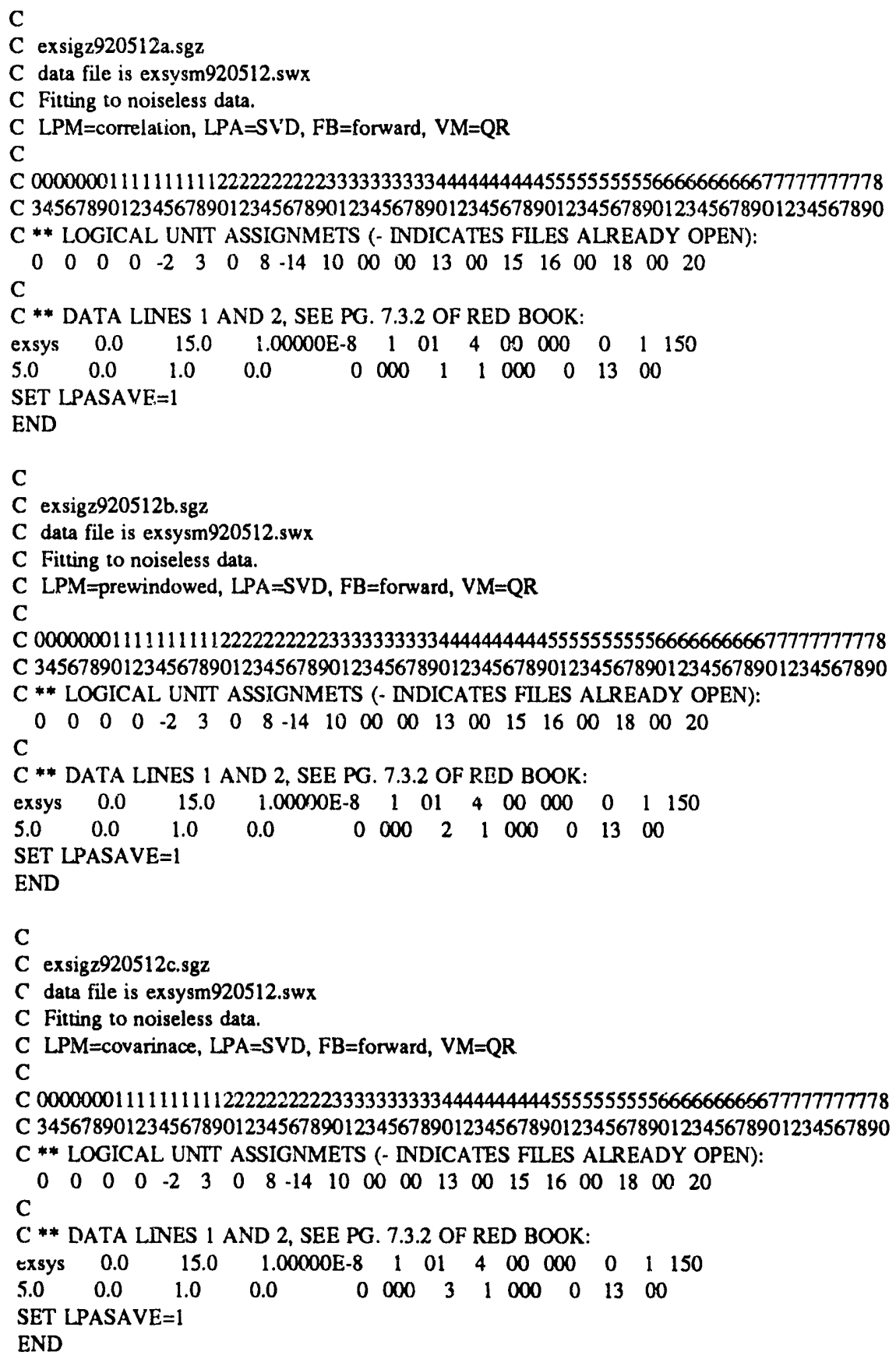






\section{Example 4.2}

C

C exsigz920513_f.sgz

C data file is exsysm920512.swx

C Fituing to noisy data (var=10)

C $L P M=$ covariance, $L P A=S V D, F B=$ backward, $V M=Q R$

$\mathrm{C}$

C 00000001111111111222222222233333333334444444444455555555556666666666777777777778

C 345678901234567890123456789012345678901234567890123456789012345678901234567890

$C$ ** LOGICAL UNIT ASSIGNMETS (- INDICATES FILES ALREADY OPEN):

$\begin{array}{llllllllllllllllllll}0 & 0 & 0 & 0 & -2 & 3 & 0 & 8 & -14 & 10 & 00 & 00 & 13 & 00 & 15 & 16 & 00 & 18 & 00 & 20\end{array}$

C

C * DATA LINES 1 AND 2, SEE PG. 7.3.2 OF RED BOOK:

$\begin{array}{llllllllllll}\text { exsys } & 0.0 & 15.0 & 0.00000 \mathrm{E}-8 & 1 & 03 & 4 & 00 & 000 & 0 & 1 & 150\end{array}$

$\begin{array}{llllllllllll}5.0 & 0.0 & 1.0 & 0.0 & 0 & 000 & 3 & 3 & 000 & 0 & 13 & 00\end{array}$

SET LPASAVE $=1$

END

C

C data file is exsysm920512.swx

C Fitting to noisy data (var=10)

C $L P M=$ covariance, $L P A=S V D, F B=$ forward-backward, $V M=Q R$

$\mathrm{C}$

C 0000000111111111122222222223333333333444444444455555555555666666666677777777778 C 345678901234567890123456789012345678901234567890123456789012345678901234567890 $C$ ** LOGICAL UNIT ASSIGNMETS (- INDICATES FILES ALREADY OPEN):

$\begin{array}{llllllllllllllllllll}0 & 0 & 0 & 0 & -2 & 3 & 0 & 8 & -14 & 10 & 00 & 00 & 13 & 00 & 15 & 16 & 00 & 18 & 00 & 20\end{array}$

C

C * DATA LINES 1 AND 2, SEE PG. 7.3.2 OF RED BOOK:

$\begin{array}{llllllllllll}\text { exsys } & 0.0 & 15.0 & 0.00000 \mathrm{E}-8 & 1 & 03 & 4 & 00 & 000 & 0 & 1 & 150\end{array}$

$\begin{array}{llllllllllll}5.0 & 0.0 & 1.0 & 0.0 & 0 & 000 & 3 & 2 & 000 & 0 & 13 & 00\end{array}$

SET LPASAVE $=1$

END

C

C data file is exsysm920512.swx

C Fitting to noisy data (var=10).

C $L P M=$ covariance, $L P A=S V D, F B=$ forward, $V M=Q R$

C

C 0000000111111111122222222223333333333344444444445555555555666666666677777777778

C 345678901234567890123456789012345678901234567890123456789012345678901234567890 
C ** LOGICAL UNIT ASSIGNMETS (- INDICATES FILES ALREADY OPEN):

$\begin{array}{llllllllllllllllllll}0 & 0 & 0 & 0 & -2 & 3 & 0 & 8 & -14 & 10 & 00 & 00 & 13 & 00 & 15 & 16 & 00 & 18 & 00 & 20\end{array}$

C

C ** DATA LINES 1 AND 2, SEE PG. 7.3.2 OF RED BOOK:

$\begin{array}{llllllllllll}\text { exsys } & 0.0 & 15.0 & 1.00000 \mathrm{E}-8 & 1 & 03 & 4 & 00 & 000 & 0 & 1 & 150\end{array}$

$\begin{array}{llllllllllll}5.0 & 0.0 & 1.0 & 0.0 & 0 & 000 & 3 & 1 & 000 & 0 & 13 & 00\end{array}$

SET LPASAVE $=1$

END

\section{Example 4.3}

C

C data file is exsysm920512.swx

C Fitting to noisy data (var=1)

C Fitting to different model orders.

C

C 0000000111111111222222222233333333333444444444455555555556666666666777777777778

C 345678901234567890123456789012345678901234567890123456789012345678901234567890

$C$ ** LOGICAL UNIT ASSIGNMETS (- INDICATES FILES ALREADY OPEN):

$\begin{array}{llllllllllllllllllll}0 & 0 & 0 & 0 & -2 & 3 & 0 & 8 & -14 & 10 & 00 & 00 & 13 & 00 & 15 & 16 & 00 & 18 & 00 & 20\end{array}$

$\mathrm{C}$

C ** DATA LINES 1 AND 2, SEE PG. 7.3.2 OF RED BOOK:

$\begin{array}{llllllllllll}\text { exsys } & 0.0 & 15.0 & 0.00000 \mathrm{E}-8 & 1 & 02 & 4 & 00 & 000 & 0 & 1 & 150\end{array}$

$\begin{array}{llllllllllll}5.0 & 0.0 & 1.0 & 0.0 & 0 & 003 & 3 & 1 & 003 & 0 & 13 & 00\end{array}$

SET LPASAVE $=1$

END

C

C data file is exsysm920512.swx

C Fitting to noisy data (var=1)

C Fitting to different model orders.

C

C 00000001111111111222222222233333333334444444444455555555556666666666677777777778 C 345678901234567890123456789012345678901234567890123456789012345678901234567890 $C$ ** LOGICAL UNIT ASSIGNMETS (- INDICATES FILES ALREADY OPEN):

C

$\begin{array}{llllllllllllllllllll}0 & 0 & 0 & 0 & -2 & 3 & 0 & 8 & -14 & 10 & 00 & 00 & 13 & 00 & 15 & 16 & 00 & 18 & 00 & 20\end{array}$

C ** DATA LINES 1 AND 2, SEE PG. 7.3.2 OF RED BOOK:

$\begin{array}{llllllllllll}\text { exsys } & 0.0 & 15.0 & 0.00000 \mathrm{E}-8 & 1 & 02 & 4 & 00 & 000 & 0 & 1 & 150\end{array}$

$\begin{array}{llllllllllll}5.0 & 0.0 & 1.0 & 0.0 & 0 & 015 & 3 & 1 & 015 & 0 & 13 & 00\end{array}$

SET LPASAVE $=1$

END

C

C data file is exsysm920512.swx

C Fitting to noisy data (var=1)

C Fitting to different model orders.

C

C 0000000111111111122222222223333333333444444444445555555555666666666677777777778

C 345678901234567890123456789012345678901234567890123456789012345678901234567890

C ** LOGICAL UNIT ASSIGNMETS (- INDICATES FILES ALREADY OPEN):

C

$\begin{array}{llllllllllllllllllllllllllll}0 & 0 & 0 & 0 & -2 & 3 & 0 & 8 & -14 & 10 & 00 & 00 & 13 & 00 & 15 & 16 & 00 & 18 & 00 & 20\end{array}$

C ** DATA LINES 1 AND 2, SEE PG. 7.3.2 OF RED BOOK:

$\begin{array}{llllllllllll}\text { exsys } & 0.0 & 15.0 & 0.00000 \mathrm{E}-8 & 1 & 02 & 4 & 00 & 000 & 0 & 1 & 150\end{array}$

$\begin{array}{llllllllllll}5.0 & 0.0 & 1.0 & 0.0 & 0 & 030 & 3 & 1 & 030 & 0 & 13 & 00\end{array}$

SET LPASAVE $=1$

END 


\section{Example 4.4}

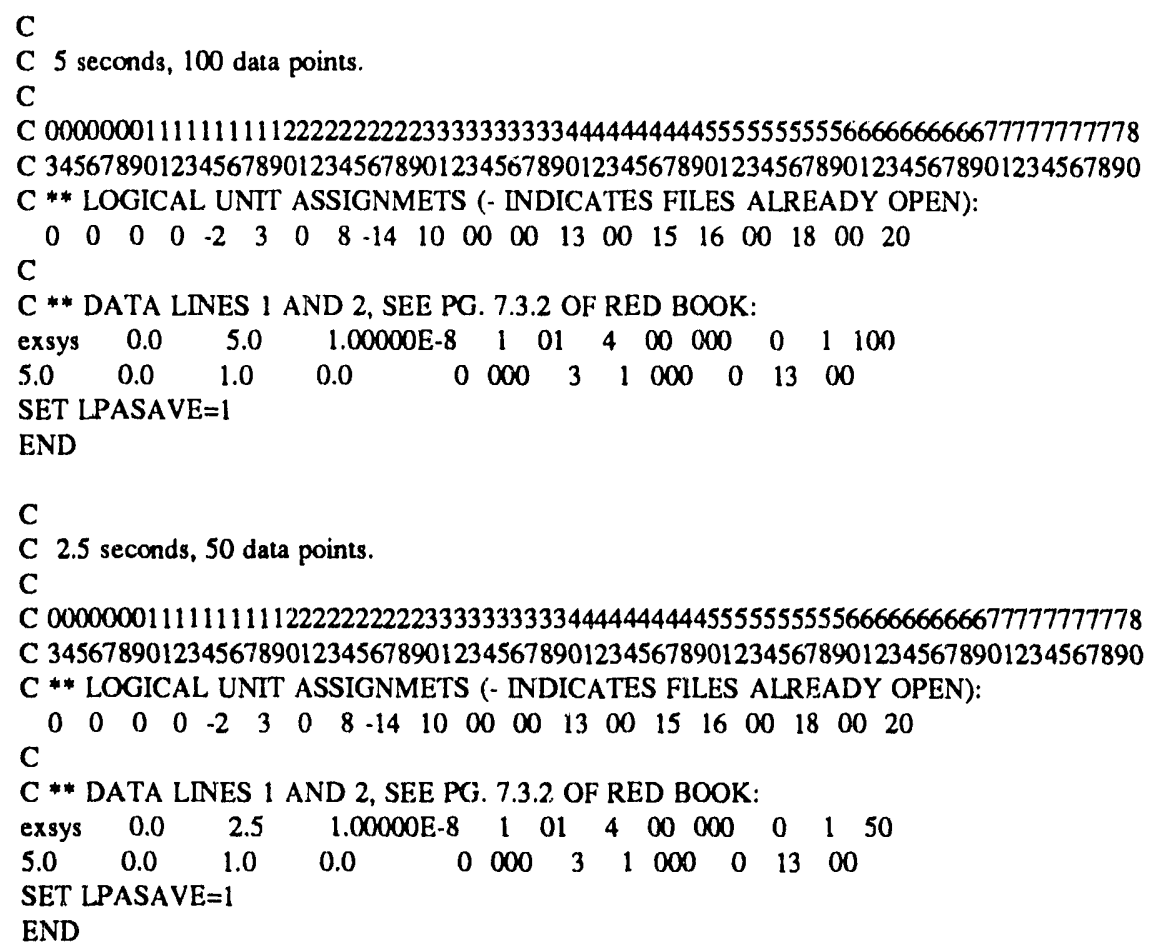

\section{Example 4.5}

$$
\text { C }
$$

C exsigz920528f.sgz

C data file is exsysm920512d.swx

C Fitting to different data record lengths of noisy data.

C noise is winois 1 (var=10)

C $L P M=$ covariance, $L P A=S V D, F B=$ forward, $V M=Q R$

C 50 seconds, 500 data points.

C

C 00000001111111111222222222233333333333444444444455555555556666666666677777777778 C 345678901234567890123456789012345678901234567890123456789012345678901234567890 C ** LOGICAL UNIT ASSIGNMETS (- INDICATES FILES ALREADY OPEN):

$\begin{array}{llllllllllllllllllll}0 & 0 & 0 & 0 & -2 & 3 & 0 & 8 & -14 & 10 & 00 & 00 & 13 & 00 & 15 & 16 & 00 & 18 & 00 & 20\end{array}$

C

C ** DATA LINES 1 AND 2, SEE PG. 7.3.2 OF RED BOOK:

$\begin{array}{llllllllllll}\text { exsys } & 0.0 & 50.0 & 0.00000 \mathrm{E}-8 & 1 & 03 & 4 & 00 & 000 & 0 & 2 & 500\end{array}$

$\begin{array}{llllllllllll}5.0 & 0.0 & 1.0 & 0.0 & 0 & 000 & 3 & 1 & 124 & 0 & 13 & 00\end{array}$

SET LPASAVE $=4$

SET VDMSAVE $=2$

END

C

C 10 seconds, 100 data points.

C

C 000000011111111112222222222333333333334444444444455555555556666666666677777777778 


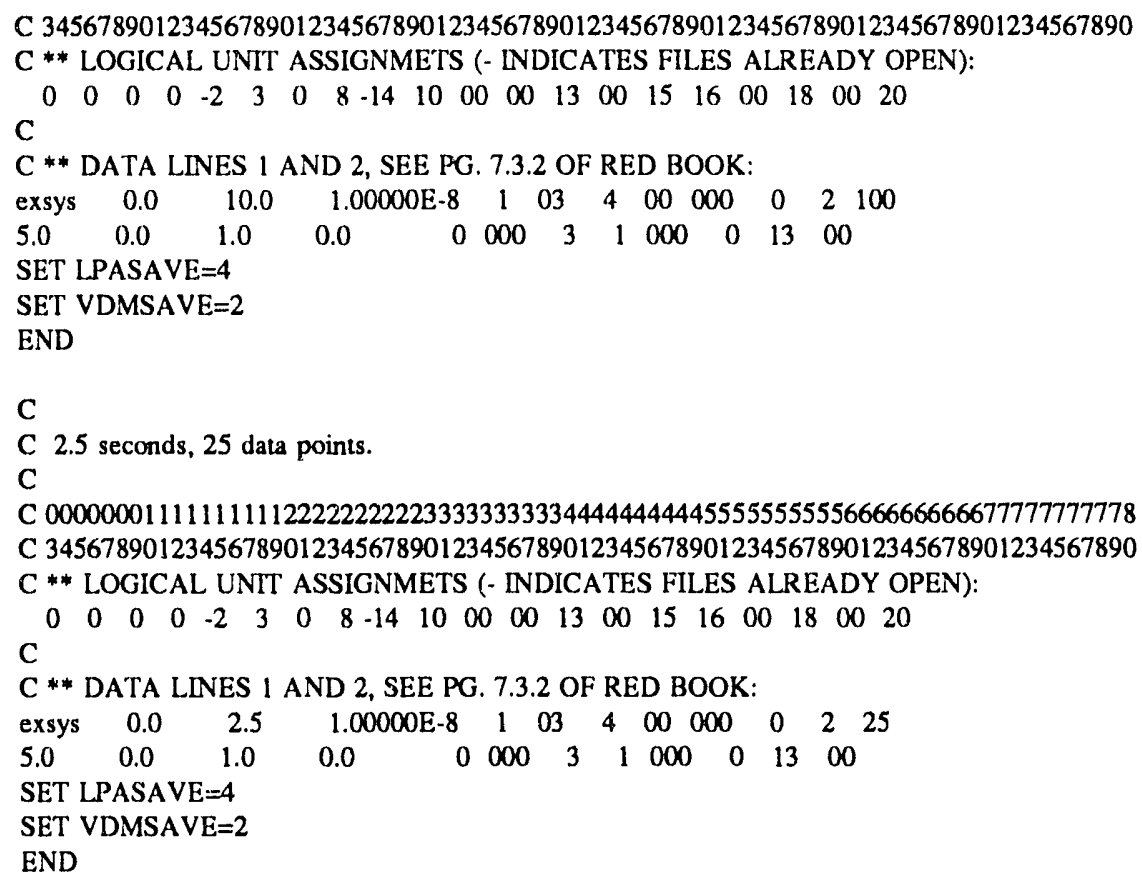

\section{Example 4.6}

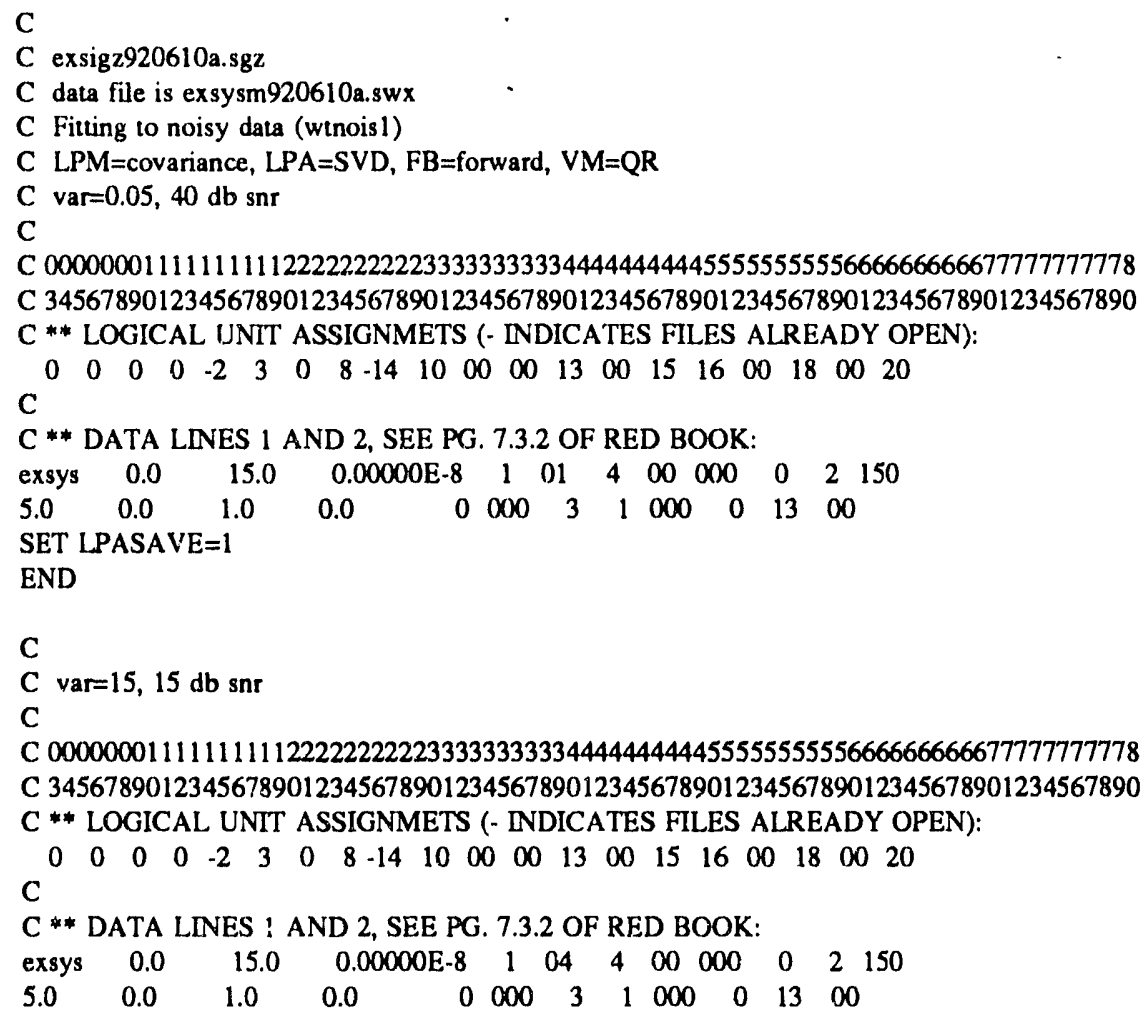


END

\section{Example 4.7}

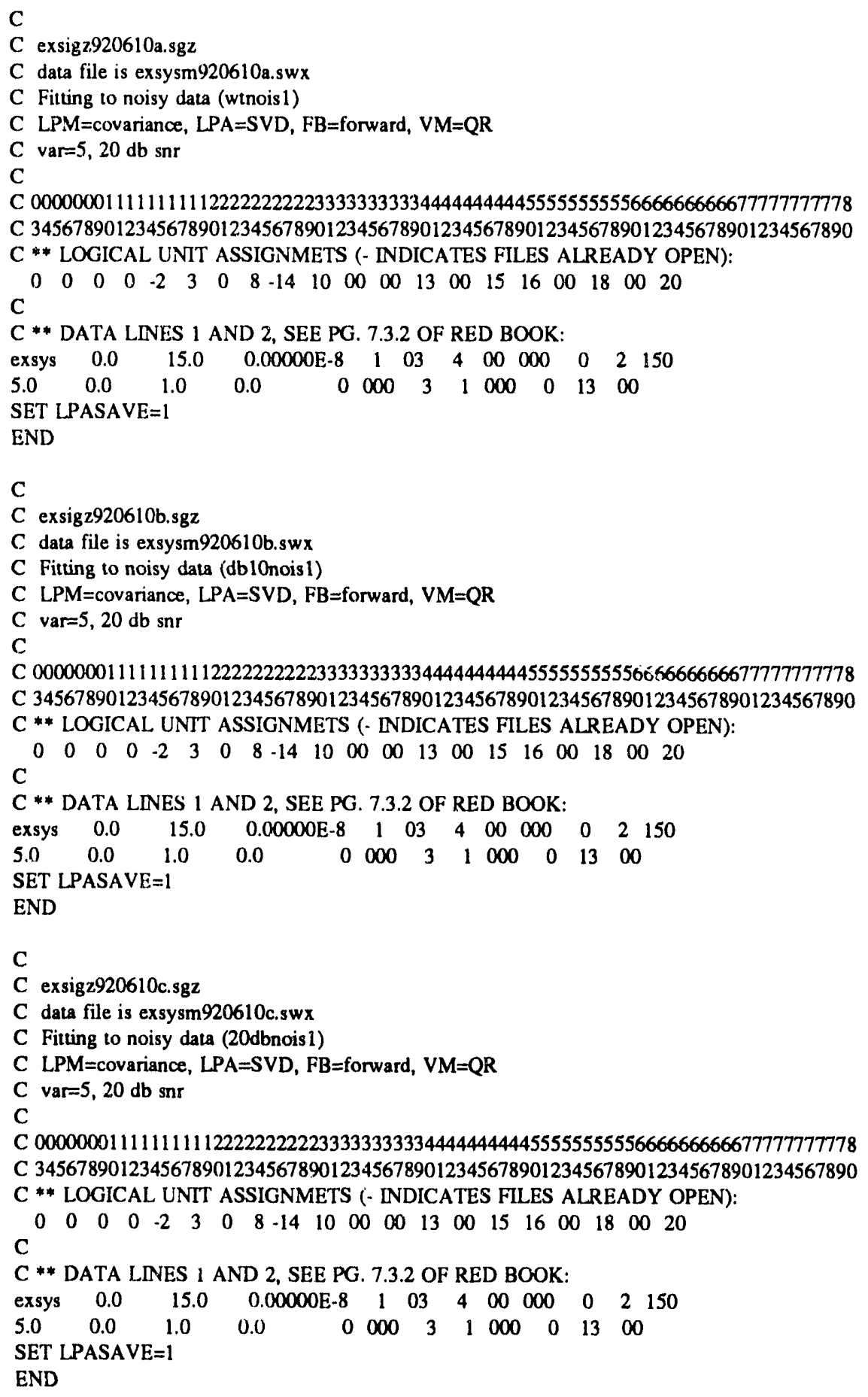


C

C exsigz920610d.sgz

C data file is exsysm920610d.swx

C Fitting to noisy data (wtnois 1 )

C $L P M=$ covariance, $L P A=S V D, F B=$ forward, $V M=Q R$

C var $=5,20 \mathrm{db}$ snr

C

C 00000001111111111222222222233333333334444444444555555555556666666666777777777778

C 345678901234567890123456789012345678901234567890123456789012345678901234567890

C ** LOGICAL UNIT ASSIGNMETS (- INDICATES FILES ALREADY OPEN):

$\begin{array}{llllllllllllllllllll}0 & 0 & 0 & 0 & -2 & 3 & 0 & 8 & -14 & 10 & 00 & 00 & 13 & 00 & 15 & 16 & 00 & 18 & 00 & 20\end{array}$

C

C ** DATA LINES 1 AND 2, SEE PG. 7.3.2 OF RED BOOK:

$\begin{array}{llllllllllll}\text { exsys } & 0.0 & 15.0 & 0.00000 \mathrm{E}-8 & 1 & 03 & 4 & 00 & 000 & 0 & 2 & 150\end{array}$

$\begin{array}{llllllllllll}5.0 & 0.0 & 1.0 & 0.0 & 0 & 000 & 3 & 1 & 000 & 0 & 13 & 00\end{array}$

SET LPASAVE $=1$

END

\section{Example 4.8}

C

C exsigz920603c.sgz

C data file is exsysm $920603 \mathrm{~b}$.swx

C Poles moving close together

C $L P M=$ covariance, $L P A=S V D, F B=$ forward, $V M=Q R$

C $\mathrm{p}=0.75$

C

C 00000001111111111222222222233333333334444444444455555555556666666666777777777778

C 345678901234567890123456789012345678901234567890123456789012345678901234567890

C ** LOGICAL UNIT ASSIGNMETS (- INDICATES FILES ALREADY OPEN):

C

$\begin{array}{llllllllllllllllllll}0 & 0 & 0 & 0 & -2 & 3 & 0 & 8 & -14 & 10 & 00 & 00 & 13 & 00 & 15 & 16 & 00 & 18 & 00 & 20\end{array}$

C ** DATA LINES 1 AND 2, SEE PG. 7.3.2 OF RED BOOK:

$\begin{array}{llllllllllll}\text { exsys } & 0.0 & 10.0 & 0.00000 \mathrm{E}-8 & 1 & 01 & 4 & 00 & 000 & 0 & 2 & 100\end{array}$

$\begin{array}{llllllllllll}5.0 & 0.0 & 1.0 & 0.0 & 0 & 000 & 3 & 1 & 000 & 0 & 13 & 00\end{array}$

SET LPASAVE $=1$

END

$\mathrm{C}$

C $\mathrm{p}=0.9$

$\mathrm{C}$

C $00000001111111111222222222233333333333444444444455555555556666666660 / 77777777778$ C 345678901234567890123456789012345678901234567890123456789012345678901234567890

C ** LOGICAL UNIT ASSIGNMETS (- INDICATES FILES ALREADY OPEN):

$\begin{array}{llllllllllllllllllll}0 & 0 & 0 & 0 & -2 & 3 & 0 & 8 & -14 & 10 & 00 & 00 & 13 & 00 & 15 & 16 & 00 & 18 & 00 & 20\end{array}$

C

C ** DATA LINES 1 AND 2, SEE PG. 7.3.2 OF RED BOOK:

$\begin{array}{lcccccccccccc}\text { exsys } & 0.0 & 10.0 & 0.00000 \mathrm{E}-8 & 1 & 02 & 4 & 00 & 000 & 0 & 2 & 100 \\ 5.0 & 0.0 & 1.0 & 0.0 & 0 & 000 & 3 & 1 & 000 & 0 & 13 & 00\end{array}$

$\begin{array}{lll}5.0 & 0.0 & 1.0 \\ \text { SET LPASAVE } & =1\end{array}$

END

C

C $\mathrm{p}=0.0$

$\mathrm{C}$

C 00000001111111111222222222233333333334444444444555555555556666666666777777777778

C 345678901234567890123456789012345678901234567890123456789012345678901234567890

C ** LOGICAL UNIT ASSIGNMETS (- INDICATES FILES ALREADY OPEN):

B.7 
C

C ** DATA LINES 1 AND 2, SEE PG. 7.3.2 OF RED BOOK:

$\begin{array}{llllllllllll}\text { exsys } & 0.0 & 10.0 & 0.00000 \mathrm{E}-8 & 1 & 04 & 4 & 00 & 000 & 0 & 2 & 100\end{array}$

$\begin{array}{llllllllllll}5.0 & 0.0 & 1.0 & 0.0 & 0 & 000 & 3 & 1 & 000 & 0 & 13 & 00\end{array}$

SET LPASAVE $=1$

END

\section{Example 4.9}

C

C exsigz920608c.sgz

C data file is exsysm920212b_2.swx

C Pole/zero pair moving close together with same frequency and noise.

C $L P M=$ covariance, $L P A=S V D, F B=$ forward, $V M=Q R$

C $\mathrm{p}=0.0$

$\mathrm{C}$

C 00000001111111111222222222233333333333444444444455555555556666666666777777777778 C 345678901234567890123456789012345678901234567890123456789012345678901234567890 $C$ ** LOGICAL UNIT ASSIGNMETS (- INDICATES FILES ALREADY OPEN):

C

$\begin{array}{llllllllllllllllllll}0 & 0 & 0 & 0 & -2 & 3 & 0 & 8 & -14 & 10 & 00 & 00 & 13 & 00 & 15 & 16 & 00 & 18 & 00 & 20\end{array}$

C ** DATA LINES 1 AND 2, SEE PG. 7.3.2 OF RED BOOK:

$\begin{array}{llllllllllll}\text { exsys } & 0.0 & 10.0 & 0.00000 \mathrm{E}-8 & 1 & 01 & 4 & 00 & 000 & 0 & 2 & 100\end{array}$

$\begin{array}{llllllllllll}5.0 & 0.0 & 1.0 & 0.0 & 0 & 000 & 3 & 1 & 000 & 0 & 13 & 00\end{array}$

SET LPASAVE $=1$

END

$\mathrm{C}$

C $p=0.75$

$\mathrm{C}$

C 00000001111111111222222222233333333334444444444455555555556666666666777777777778 C 345678901234567890123456789012345678901234567890123456789012345678901234567890 C ** LOGICAL UNIT ASSIGNMETS (- INDICATES FILES ALREADY OPEN):

$\begin{array}{llllllllllllllllllll}0 & 0 & 0 & 0 & -2 & 3 & 0 & 8 & -14 & 10 & 00 & 00 & 13 & 00 & 15 & 16 & 00 & 18 & 00 & 20\end{array}$

C

C ** DATA LINES 1 AND 2, SEE PG. 7.3.2 OF RED BOOK:

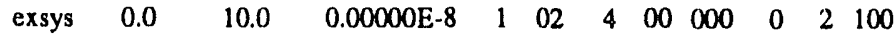

$\begin{array}{llllllllllll}5.0 & 0.0 & 1.0 & 0.0 & 0 & 000 & 3 & 1 & 000 & 0 & 13 & 00\end{array}$

SET LPASAVE $=1$

END

C

C $p=0.9$

$\mathrm{C}$

C 00000001111111111222222222233333333334444444444455555555556666666666777777777778 C 345678901234567890123456789012345678901234567890123456789012345678901234567890

C ** LOGICAL UNIT ASSIGNMETS (- INDICATES FI: FS ALREADY OPEN):

C ** DATA LINES 1 AND 2, SEE PG. 7.3.2 OF RED BOOK:

$\begin{array}{lcccccccccccc}\text { ersys } & 0.0 & 10.0 & 0.00000 \mathrm{E}-8 & 1 & 03 & 4 & 00 & 000 & 0 & 2 & 100 \\ 5.0 & 0.0 & 1.0 & 0.0 & 0 & 000 & 3 & 1 & 000 & 0 & 13 & 00 & \\ \text { SET LPASAVE }=1 & & & & & & & & & & & & \end{array}$

B. 8 
Appendix C

TRANFUN and ORDER Subroutines 


\section{Appendix C}

\section{TRANFUN and ORDER Subroutines}

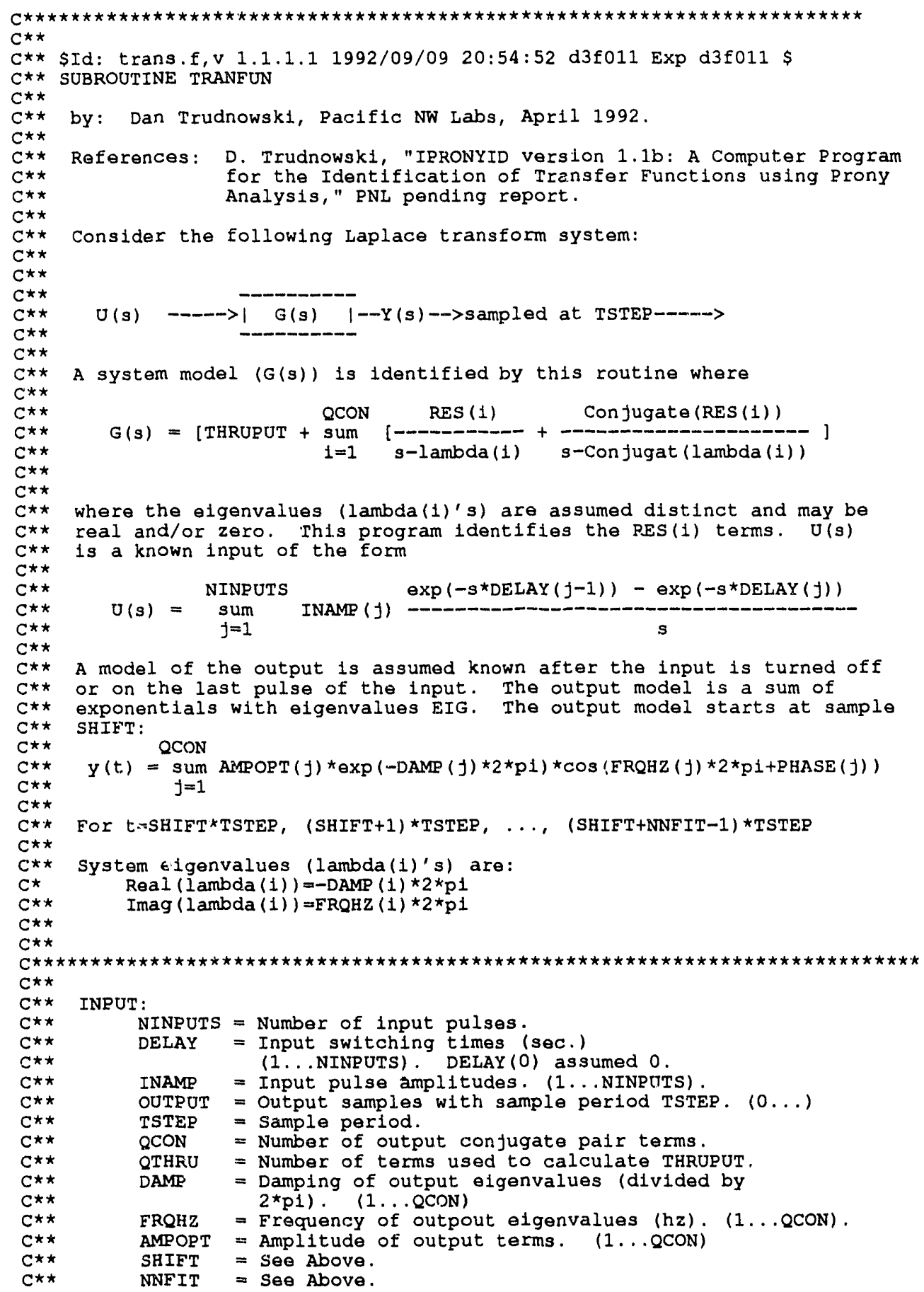




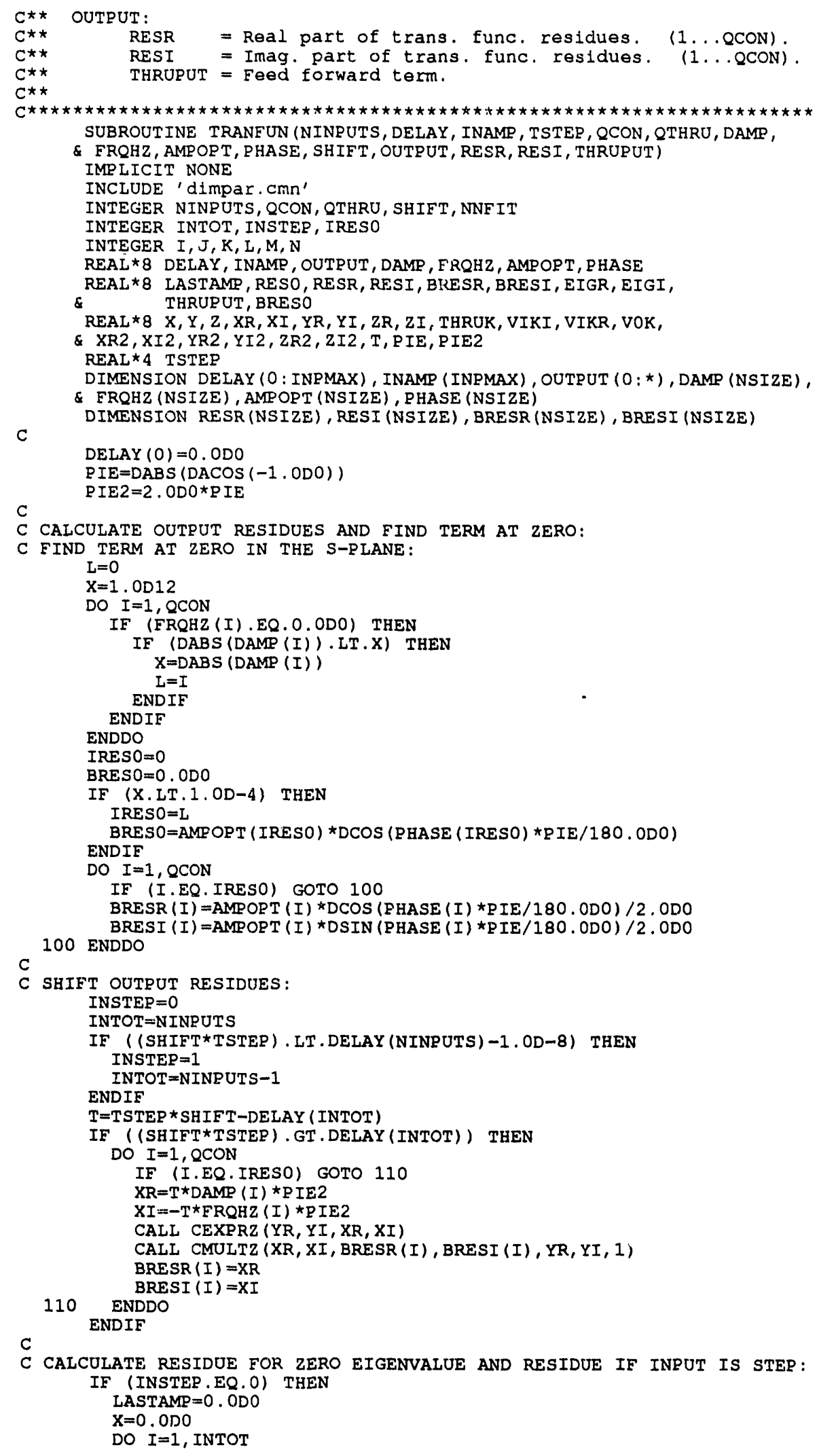




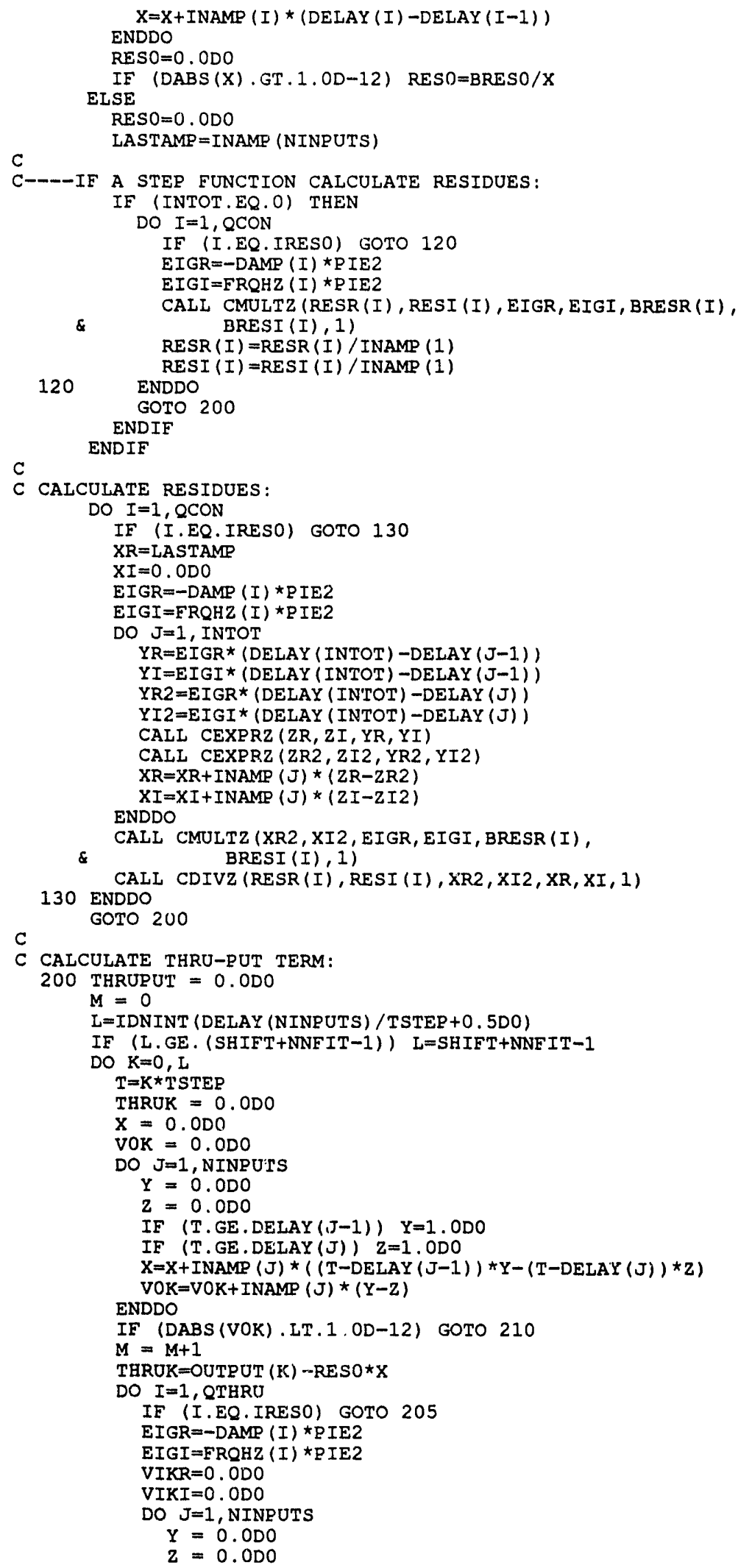




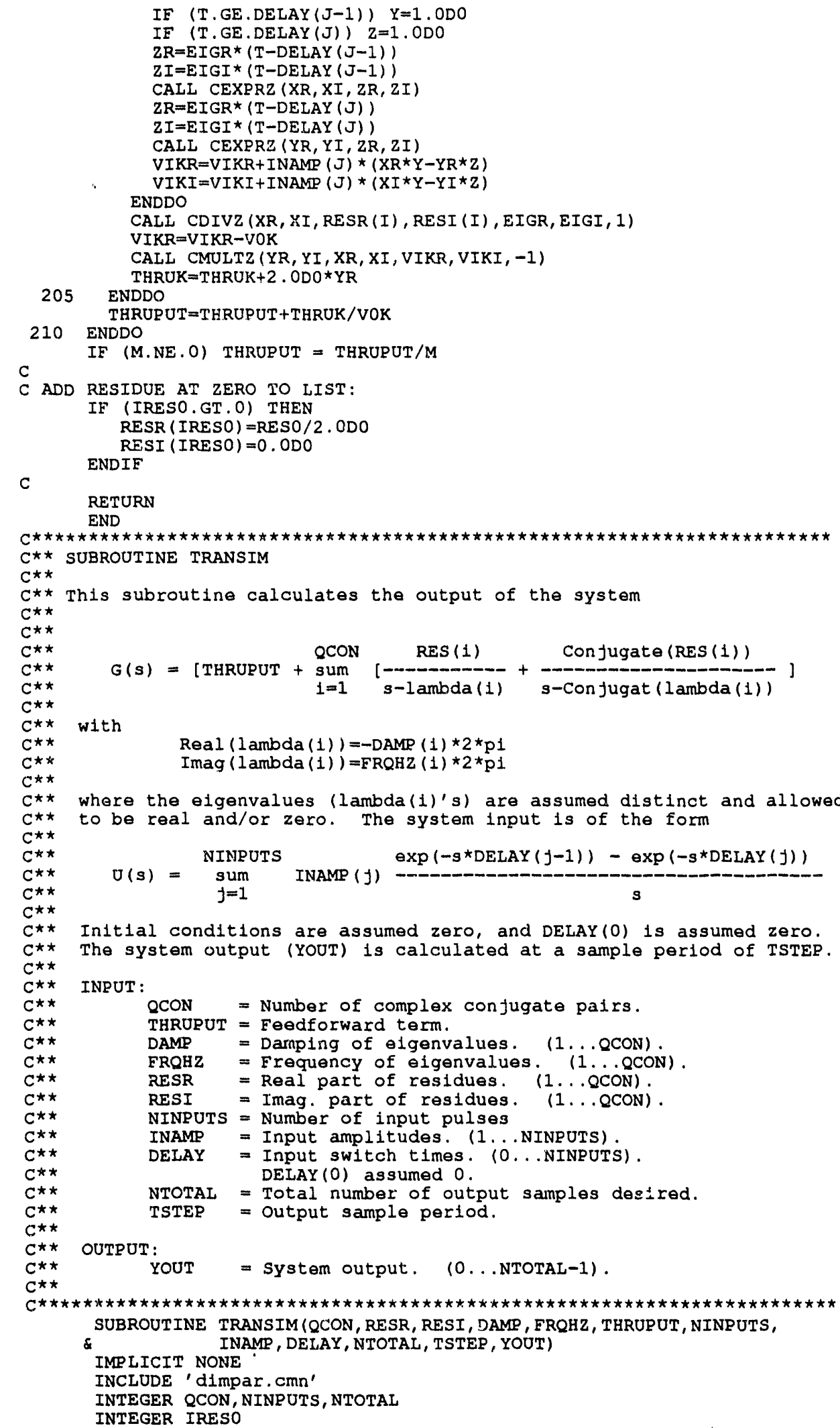




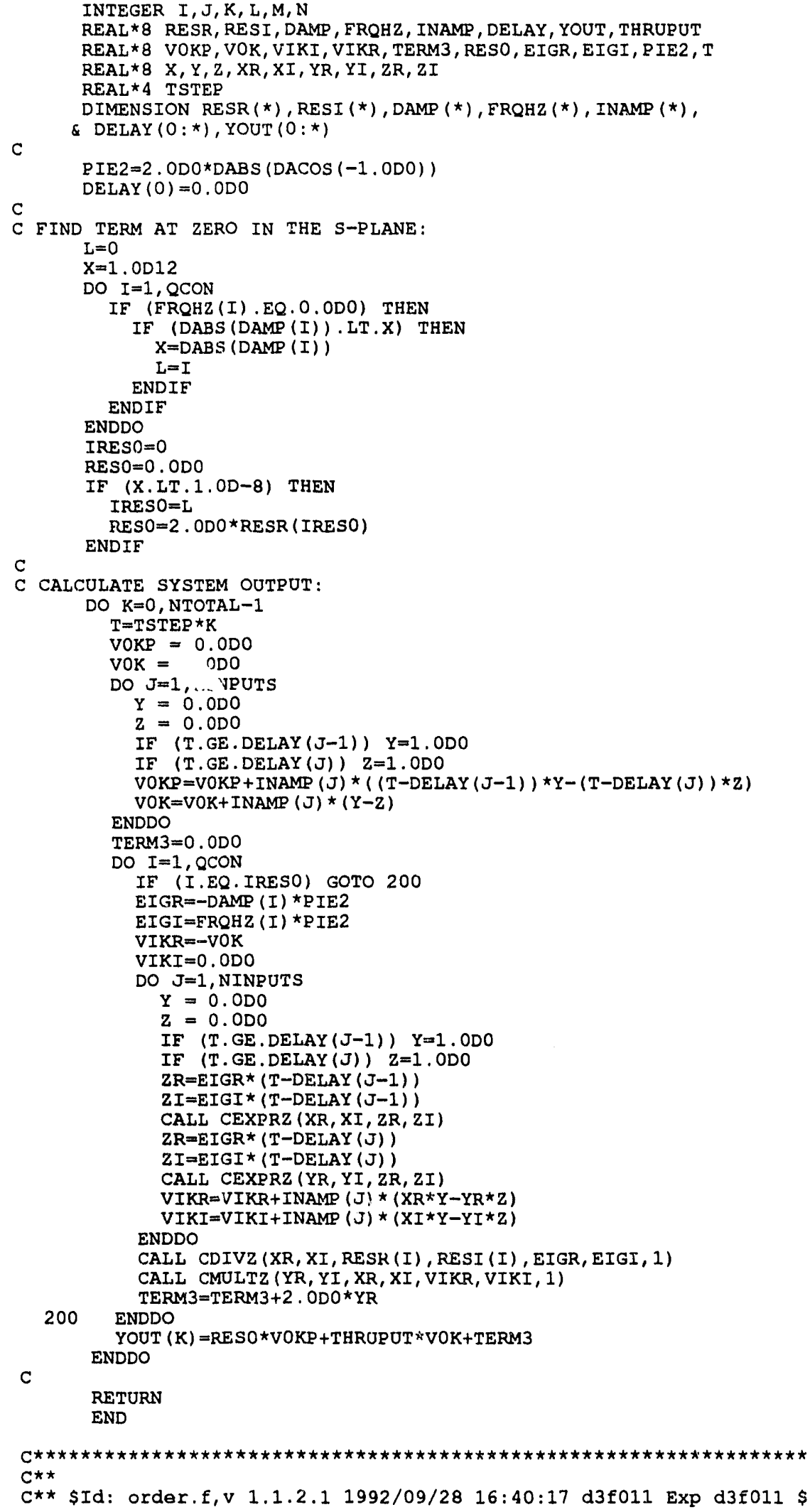




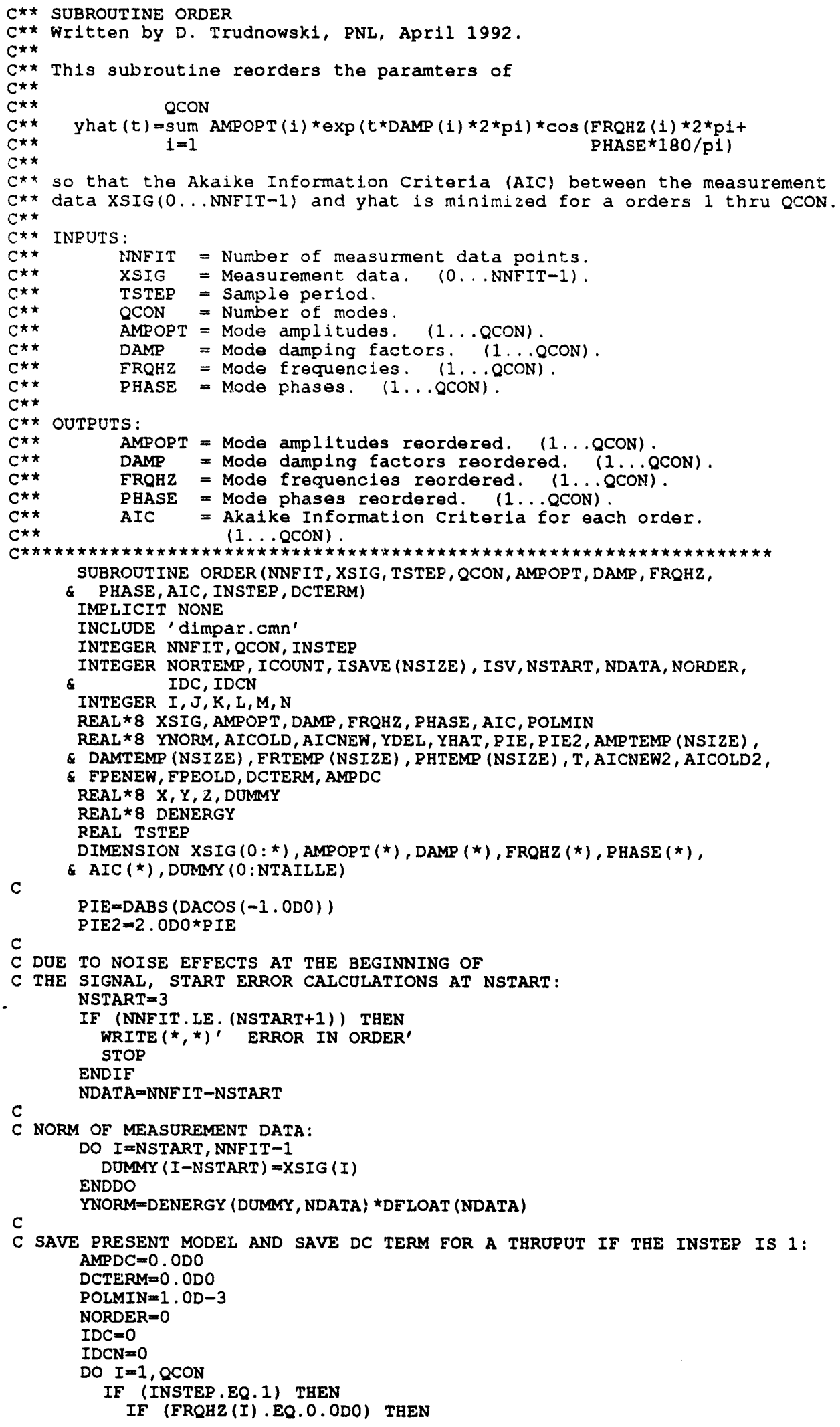




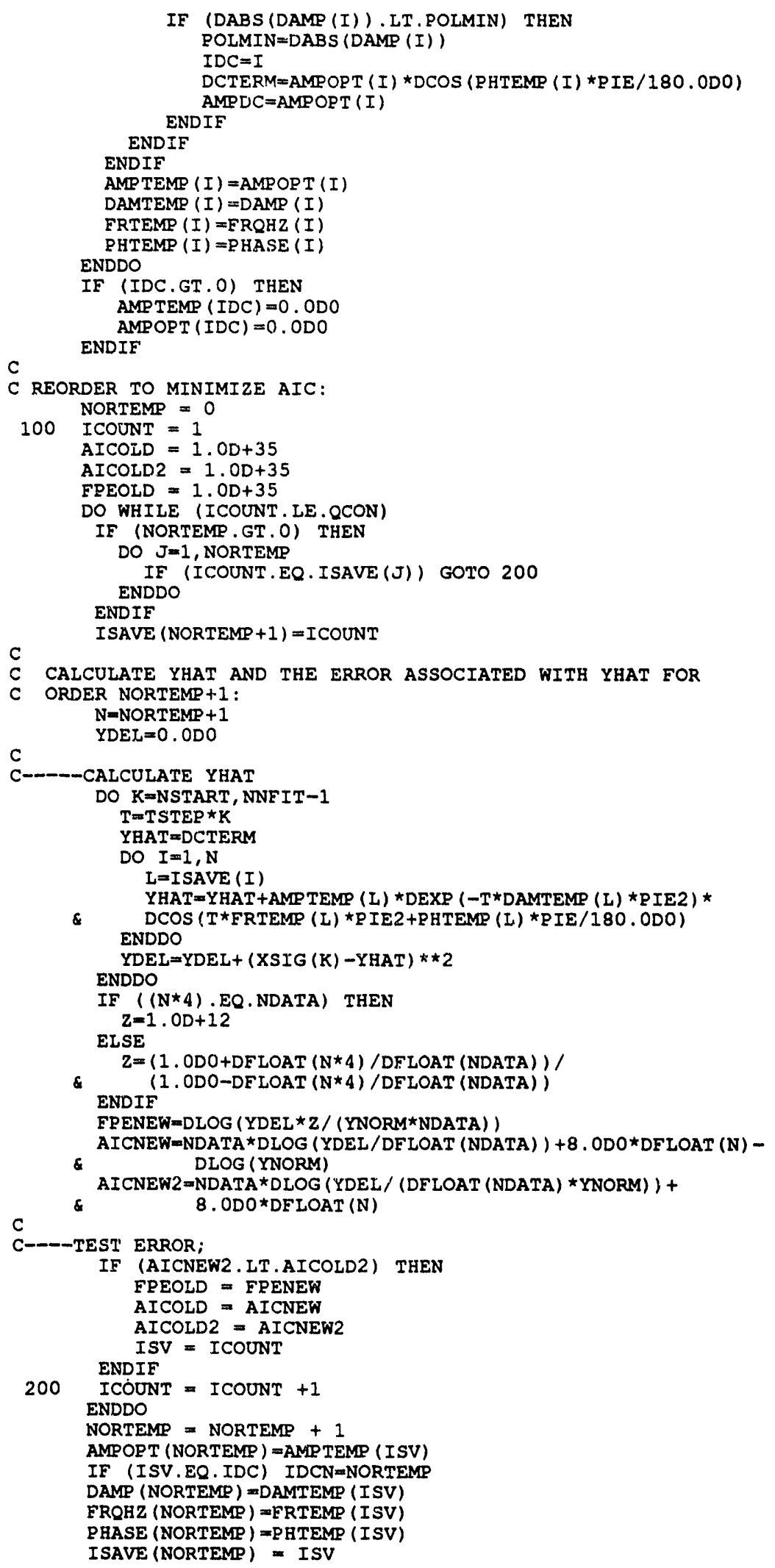


AIC (NORTEMP) = AICOLD2

IF (NORTEMP .LT. QCON) GOTO 100

C

IF (IDCN.GT.0) AMPOPT (IDCN) =AMPDC

RETURN

END

C.8 


\section{Distribution}

No. of

Copies

Offsite

12 DOE/Office of Scientific and Technical Information

2 J. F. Hauer, $\mathrm{EOH}$

Bonneville Power Administration

P.O. Box 3621

Portland, OR 97208

L. L. Scharf

Department of Electrical and Computer Engineering

Engineering Center OT 3-12

University of Colorado at Boulder

Boulder, CO 80309-0425

\section{J. Agee}

M.S. D-3772

U.S. Bureau of Reclamation

P.O. Box 25007

Denver, CO 80225

D. A. Pierre

Electrical Engineering Department

Montana State University

Bozeman, MT 59717
No. of

Copies

Onsite

Department of Energy Richland Field Office

D. R. Segna

19 Pacific Northwest Laboratory

M. K. Donnelly

D. M. Elwood

L. D. Kannberg

D. J. Trudnowski (10)

Publishing Coordination

Technical Report Files (5) 

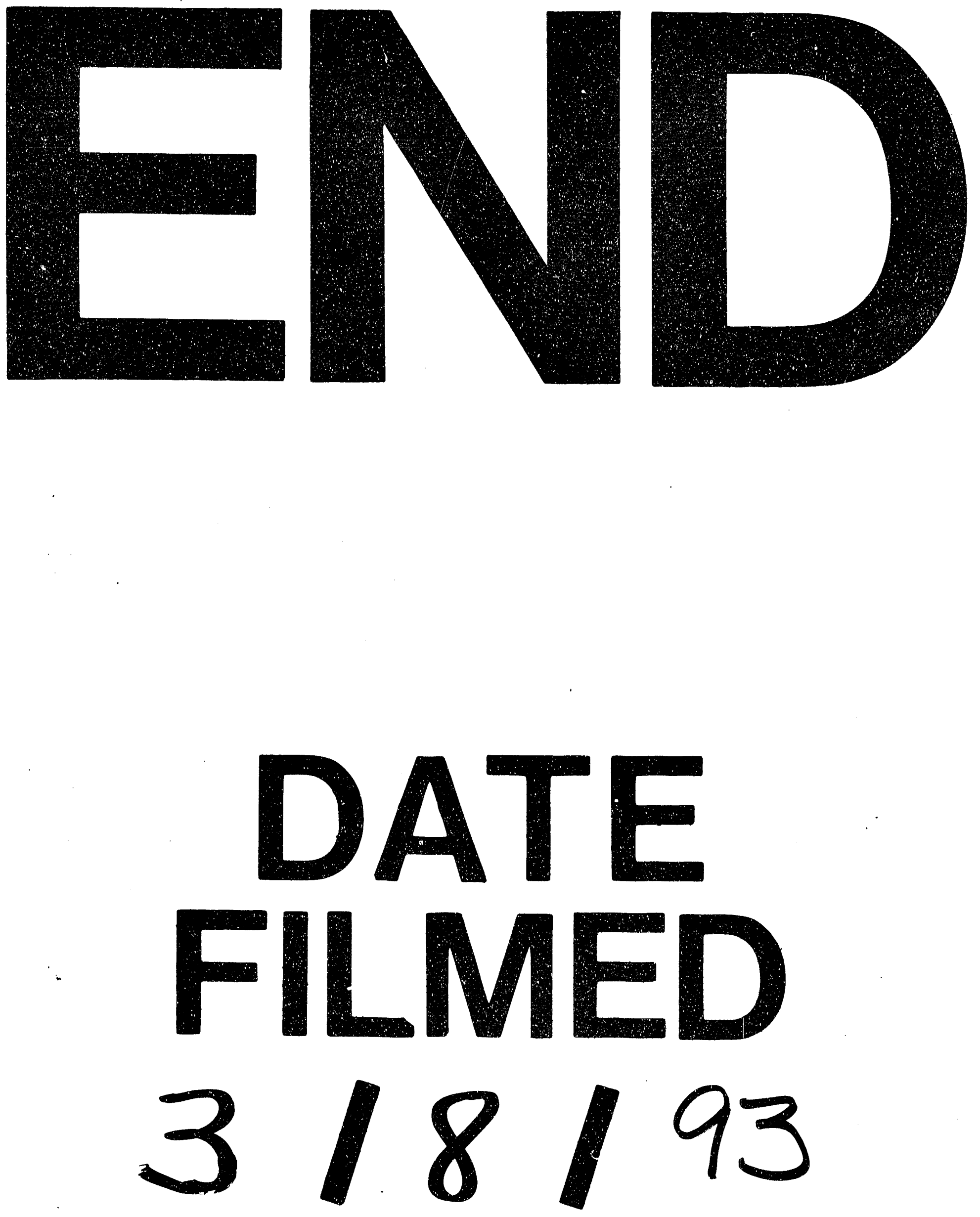
